\title{
Design of the cold and thermal neutron moderators for the European Spallation Source
}

Zanini, L.; Andersen, K. H.; Batkov, K.; Klinkby, E. B.; Mezei, F.; Schönfeldt, T.; Takibayev, A.

Published in:

Nuclear Inst. and Methods in Physics Research, A

Link to article, DOI:

10.1016/j.nima.2019.01.003

Publication date:

2019

Document Version

Peer reviewed version

Link back to DTU Orbit

Citation (APA):

Zanini, L., Andersen, K. H., Batkov, K., Klinkby, E. B., Mezei, F., Schönfeldt, T., \& Takibayev, A. (2019). Design of the cold and thermal neutron moderators for the European Spallation Source. Nuclear Inst. and Methods in Physics Research, A, 925, 33-52. https://doi.org/10.1016/j.nima.2019.01.003

\section{General rights}

Copyright and moral rights for the publications made accessible in the public portal are retained by the authors and/or other copyright owners and it is a condition of accessing publications that users recognise and abide by the legal requirements associated with these rights.

- Users may download and print one copy of any publication from the public portal for the purpose of private study or research.

- You may not further distribute the material or use it for any profit-making activity or commercial gain

- You may freely distribute the URL identifying the publication in the public portal 


\section{Accepted Manuscript}

Design of the cold and thermal neutron moderators for the European Spallation Source

L. Zanini, K.H. Andersen, K. Batkov, F. Mezei, A. Takibayev, E.B. Klinkby, T. Schönfeldt

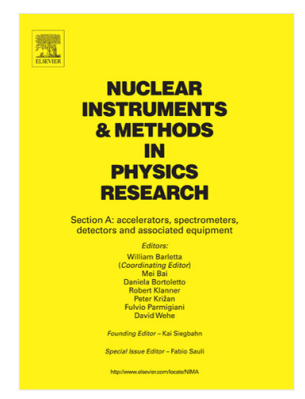

PII: $\quad$ S0168-9002(19)30008-7

DOI: $\quad$ https://doi.org/10.1016/j.nima.2019.01.003

Reference: $\quad$ NIMA 61776

To appear in: $\quad$ Nuclear Inst. and Methods in Physics Research, A

Received date : 14 August 2018

Accepted date : 2 January 2019

Please cite this article as: L. Zanini, K.H. Andersen, K. Batkov et al., Design of the cold and thermal neutron moderators for the European Spallation Source, Nuclear Inst. and Methods in Physics Research, A (2019), https://doi.org/10.1016/j.nima.2019.01.003

This is a PDF file of an unedited manuscript that has been accepted for publication. As a service to our customers we are providing this early version of the manuscript. The manuscript will undergo copyediting, typesetting, and review of the resulting proof before it is published in its final form. Please note that during the production process errors may be discovered which could affect the content, and all legal disclaimers that apply to the journal pertain. 


\title{
Design of the cold and thermal neutron mudr,rators for the European Spallation Sorice
}

\author{
L. Zanini, K. H. Andersen, K. Batkov, F. N ${ }^{\prime}$ zei. ‘ Takibayev \\ European Spallation Source ESS ERIC, PO Box 176, „2100 _und, Sweden
}

E. B. Klinkby, T. Schönfeldt

DTU Nutech, Technical University of Denmark, DTU Risø Campus, जrederıksborgvej 399, DK-4000 Roskilde, Denmark

\section{Abstract}

At the European Spallation Source (ESS), neutron xill be renerated by spallation induced by a 2-GeV proton beam on a tungsten target. ESS will have a grid of 42 beals. orts available for a variety of neutron scattering experiments. Neutron moderators will provide thermal and cold neutru.s to the instruments, allowing bispectral beam extraction wherever needed.

The moderators were designed by adopti g a hoı tic design approach that has considered brightness, brightness transfer and beam extraction constraints, re rlting i i a system with the following main features: low-dimensional moderators for enhanced brightness and iaximu. Iux to the sample; a single moderator system placed above the spallation target; lateral shape of the $\mathrm{m}$ jder tors optimized for bispectral extraction. A moderator with a vertical extraction surface of $3 \mathrm{~cm}$ was chosen $a_{i}$ 'o ult $r$. the optimization process.

With all initial instruments point $n g$ to $l_{\perp}$. op moderator, and a beamport system that allows the possibility to extract neutrons from above and $\mathrm{b} \varkappa$ the target, the adopted configuration opens the possibility to have different types of moderators below the target, so hat other neutron beams of different intensity, or spectral shape, with respect to the ones delivered by 1 te op moderator, could be envisaged, adding additional scientific opportunities to the facility without having the aeed io build a second target station.

Keywords: low-dimensiona' modera `rs, source brightness, parahydrogen, water, neutron beam extraction, long pulse sources

\section{Introduction}

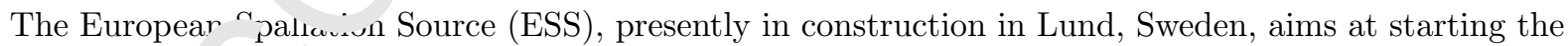
user program in 2023 [ . At $5 \mathrm{MW}$ time-average power, and $125 \mathrm{MW}$ peak power, ESS will be the most powerful neitron suuce in the world for neutron scattering studies of condensed matter. Neutrons will be produced by a ? ieV proton beam impinging on a target made of tungsten. ESS will be the first high-power accelerator driven long pulse source [2], the pulse length of the beam will be of $2.86 \mathrm{~ms}$, with $14 \mathrm{~Hz}$ repetition rate. 
Neutron moderators, placed next to the spallation target and designed to slow dow' neutrons from $\mathrm{MeV}$ to the sub-eV range, are a key component of the neutron source. While the acc eran power is a key parameter to indicate the performance of the facility, the brightness of the nev ron source, for cold and thermal neutrons, is more relevant for the neutron scattering experiments, because $\cdot$ is a quantity directly related to the number of neutrons at the sample.

The first complete moderator design was presented in the Technical ${ }^{\top}$ esig $\perp \mathrm{h} \_$port (TDR) [1] and in Ref. [3]. The TDR cold moderators were pure parahydrogen cylindrimel mu 'rators of $16 \mathrm{~cm}$ diameter and $13 \mathrm{~cm}$ height, and their calculated brightness exceeded the desig. goal oi ESS, to deliver a cold peak brightness a factor of 30 higher than the official ILL cold brightner [4]. ...iver the work for the TDR, the ESS neutronic team led a research effort aimed at designing mod $\mathrm{ra}^{+} \mathrm{urs} \mathrm{r}$ ith further increased brightness. The design of the moderators was redefined based on the concepu f low-dimensional moderators $[5,6]$. As low-dimensional moderators represent a new idea for enhanc. ${ }^{\lambda}$ modf ator brightness, we discuss in detail their physics properties in Section 2.

In this discussion we concentrate on flat, quasi-two-dn. nsional moderators, because it is the design basis for the ESS moderators. (quasi-one dimensional mod ....... are discussed in AppendixB). In Section 3 the different moderator concepts that were considered for $\mathrm{E}^{2}$; are discussed. In Section 4 the beam extraction aspects and their role on the moderator design are $a_{n}$ 'issed. The performances of the moderators are compared in Section 5. In Section 6 the butterfly- ' 'ape _..oderator, the selected moderator shape, is described and characterized in detail. Conclusions and some initial suggestions for future upgrade possibilities are given in Section 7. The method of brightness $\mathrm{c}$ dculatı $\mathrm{n}$, and the tube moderators (one of the options for future upgrades at ESS) are discussed in the ppen ${ }^{~}: \mathrm{cr}$ s.

All calculations have been perfor ed sith MCNPX 2.7.0 [7-9] using the default Bertini/Dresner spallation/evaporation models and ENJ F/B- $V_{\text {. }}$ nuclear data libraries.

\section{Properties of low-dimr asic nal moderators}

Low-dimensional mod ratnrs tw ve been proposed in recent years to increase the brightness of neutron sources. Studies on moan ors f reduced dimensions were carried out from first principles to conceptual design for two high- ower nallation sources under design: ESS $[5,6,10]$ and the SNS second target station [11]. In these 1. -ilitie a large number of beam lines covering beam extraction sectors $60^{\circ}$-wide or more is foreseer For t is purpose, two-dimensional moderators are considered. Following the more radical concept of quasi- ne-di lensional moderators [6], ideas to use tube moderators in reactors [6] and compact sources [12] ave ... a brought up, exploiting the strong brightness and directionality of such moderators, which gives an - tra advantage in specific situations where the moderator is feeding only a few beam lines covering a narrow angular span of about $10-20^{\circ}$. While possible future applications of tube moderators for 
Figure 1. Basic physics model of a pancake moderator. Left: horizontal cut. Right: vertical cut of the + rget-moderator-reflector configuration. The $2-\mathrm{GeV}$ proton beam is hitting the target perpendicular to the plane of the figure.
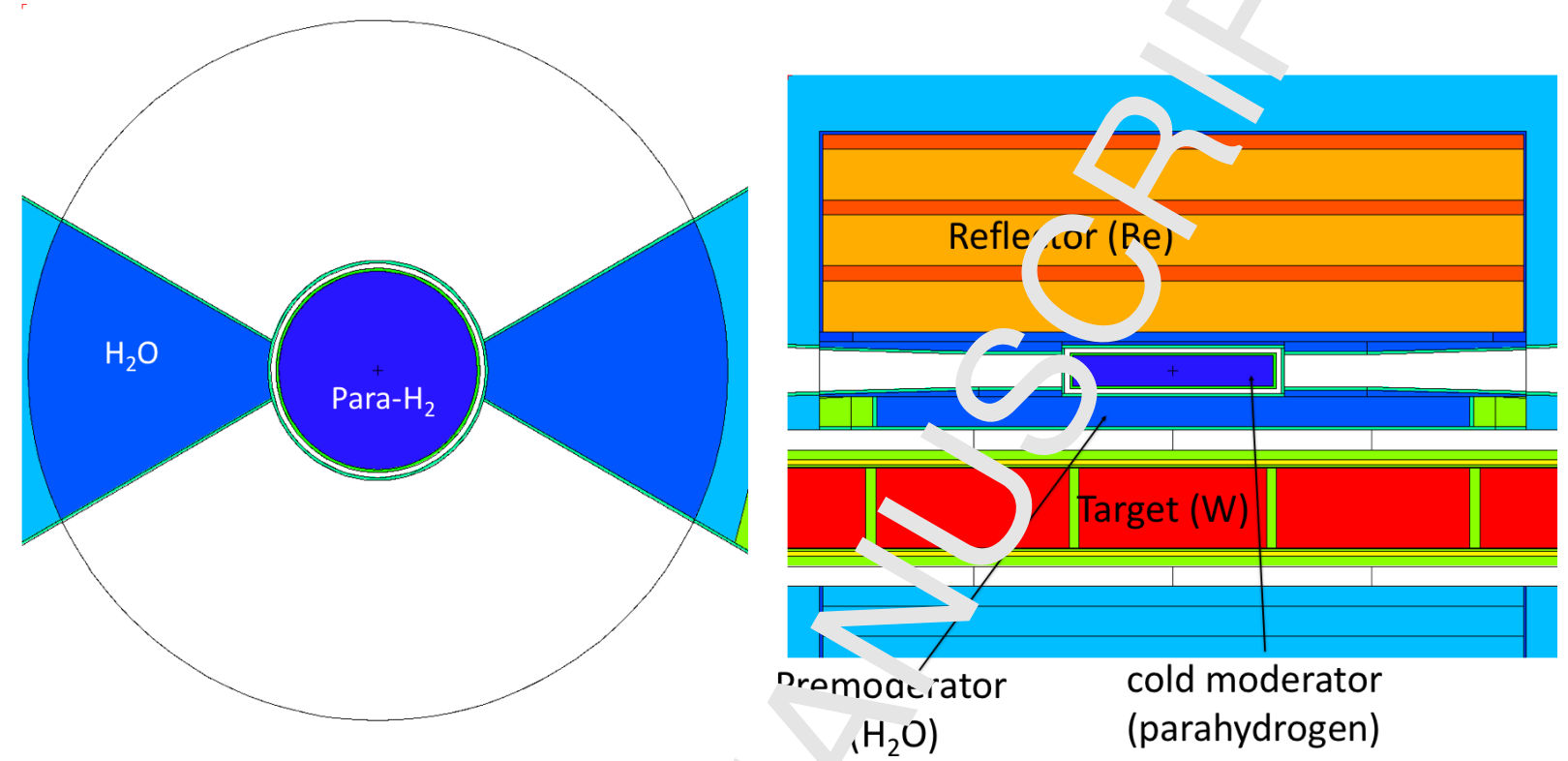

ESS are discussed in the appendix, in this $\mathrm{s}^{-1} \mathrm{n}$ n we examine the properties of two-dimensional moderators, which are the basis for the ESS design.

\subsection{Quantification of the brightness qair}

The fundamental properties $\mathrm{c}$ the lo dimensional moderators can be understood by the study of a simple quasi-two-dimensional nodera ${ }^{\boldsymbol{r}}$ of cylindrical shape, which we called pancake: a cylindrical aluminum vessel filled with purf par hydrogen, placed above a spallation target, with a beryllium reflector on top, and a water premoderato, netween target and moderator (Figure 1). The premoderator shapes the energy spectrum of the ' eutr sns, which, coming from the spallation target, have high energies in the range of 1-2 MeV. We considnr the se of neutron beam extraction over a large angular range, with two sectors covering $120^{\circ}$ each ecause his applies directly to ESS (a wider opening area is in practice not possible for a configuratir - whe une proton beam hits the target horizontally). However, the discussion can be generalized to c fferent eam extraction configurations.

We defino the brigıtness $B$ in AppendixA. Although the brightness is time-dependent, we describe it in terms of a pea - nd a time-average brightness, since it is largely constant during the duration of the proton pulse (apart from. the rise and fall-off tails). For the peak brightness, we assume that $B$ is equal to the plateau value reached during a $2.86 \mathrm{~ms}$ long pulse. For the time-average brightness, the pulse structure of 
Figure 2. Cold peak brightness wavelength spectra for moderators of different height salcula ed from the geometry of Figure 1. Neutrons are extracted in an exit direction perpendicular to the incoming p ston hoam, at the center of the $120^{\circ}$ sector of Figure 1 (left).

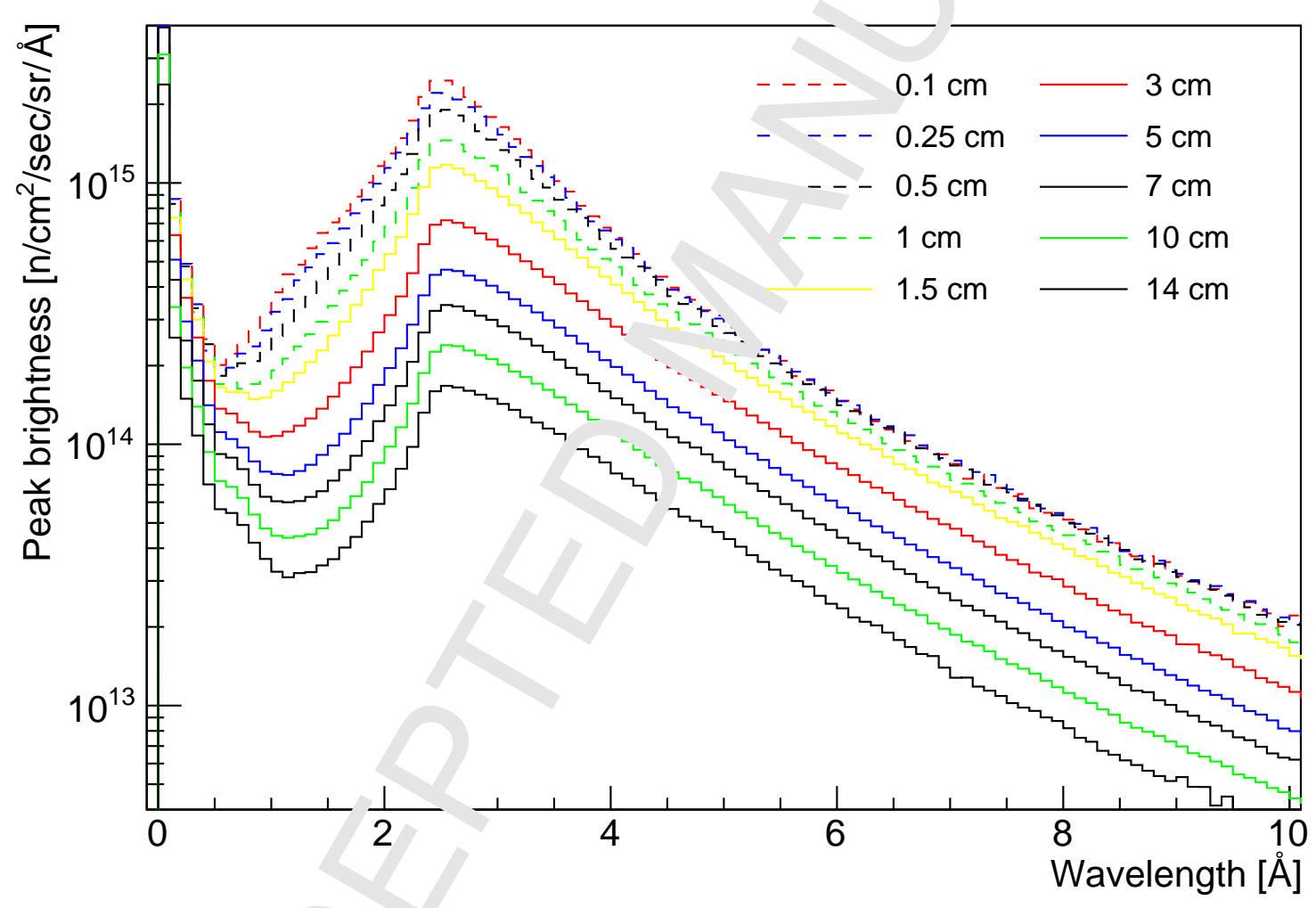


Figure 3. Same as Figure 2, for thermal neutrons, extracted $1_{\Perp} n \cdots$.ter wings.

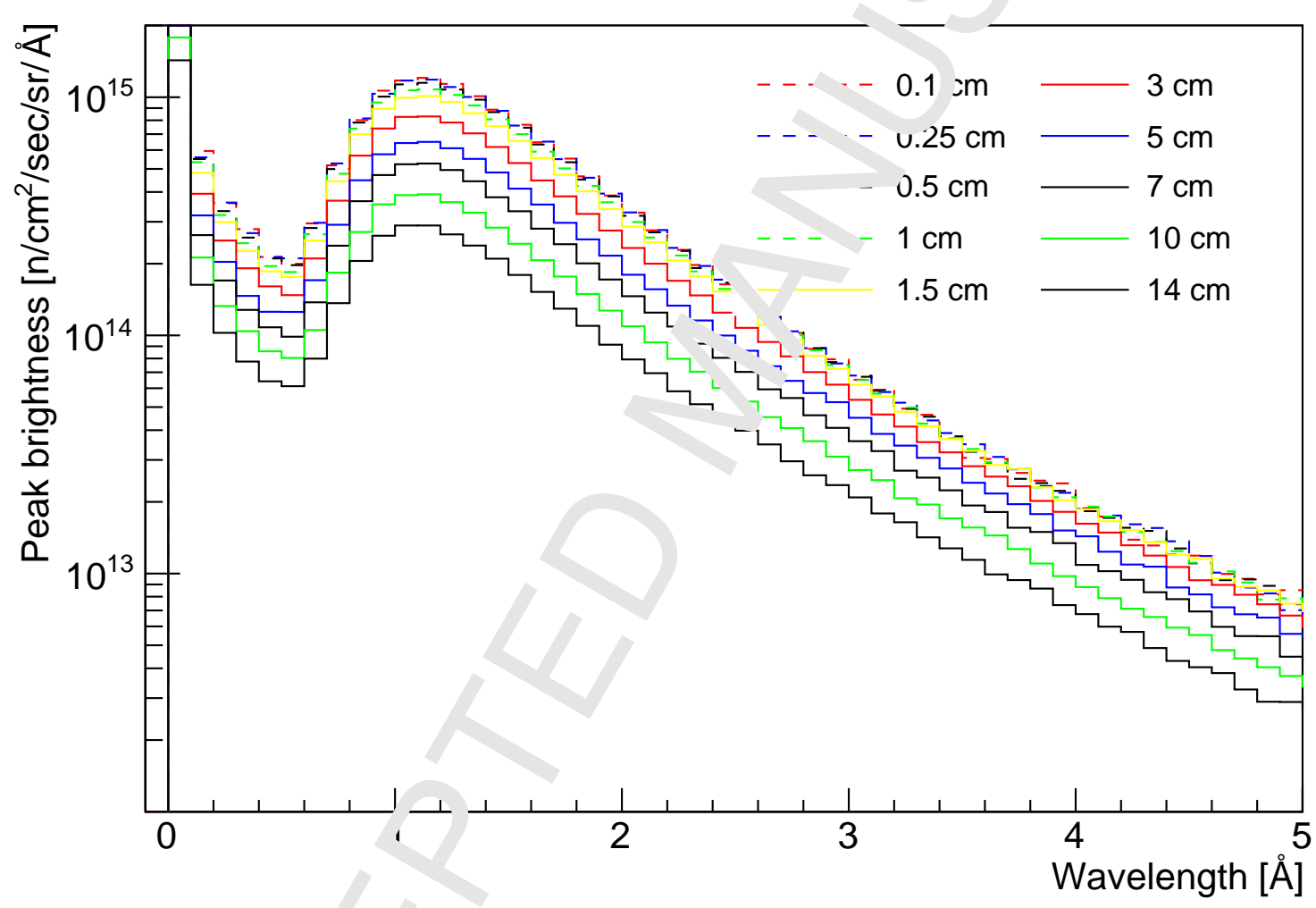


Figure 4. Cold brightness gains with respect to $10 \mathrm{~cm}$ tall moderator, from the spectra she $\eta$ in Figure 2.

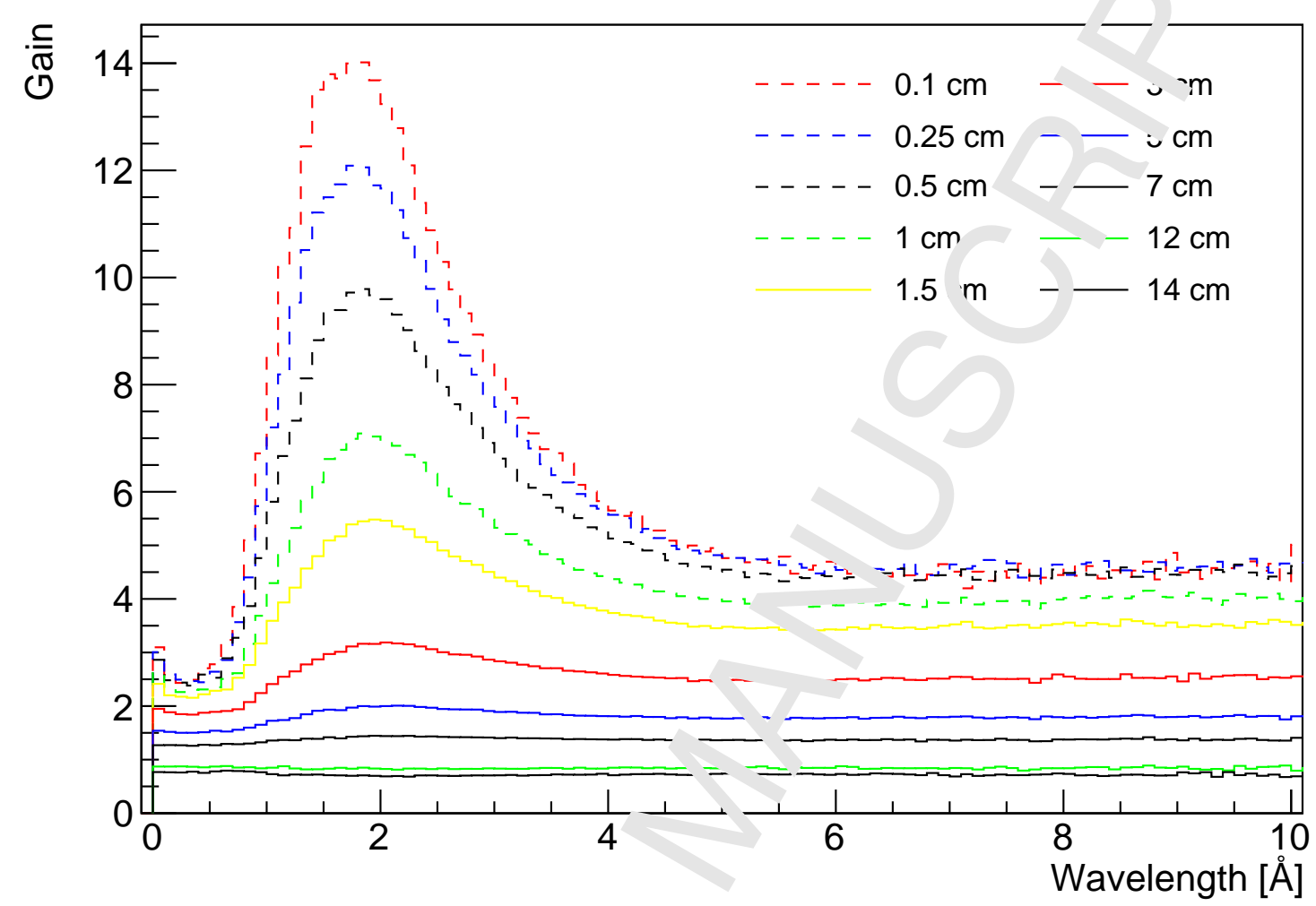

the beam is not considered and the atio petween peak and time-average brightness is $T / \tau=25$, where the repetition period $T=1 / 14 \mathrm{~Hz}$ an $r$ the ${ }_{r}{ }^{-1}$, length $\tau \approx 2.86 \mathrm{~ms}$.

In Figure 2 and Figure 3 the spe 'ral brightness from the cold and thermal parts of the moderators are shown, respectively. Cold ne .ur is are extracted from the center of the cold moderator, from an area of fixed width of $6 \mathrm{~cm}$, with hel 't equal to the height of the moderator. The brightness is averaged over the extraction surface. In thr cas' of the thermal moderator, neutrons are extracted from a window of the same area, from the water mode ${ }^{+}{ }^{\text {or }}$ placed at the side of the cold moderator of Figure 1.

The wavelength pectra, divided by the brightness of the 10-cm-tall moderator, thus representing the brightness gain as a 1 unti $\mathrm{n}$ of the wavelength relative to the $10 \mathrm{~cm}$ moderator, are shown in Figures 4 and 5. There is 1 qualit. tive difference between the gain from the thermal moderators and parahydrogen, as a function of the $w \cdots$ ngth: while for thermal neutrons the gain does not vary much within the wavelength span shown 1. Fi jure 5, a strong variation is observed for cold neutrons.

For the deter ination of the optimal height of the moderator, and in general for its neutronic design, we considered integrated cold and thermal brightness in different energy ranges of interest for most common 
Figure 5. Same as Figure 4, for thermal neitrc.

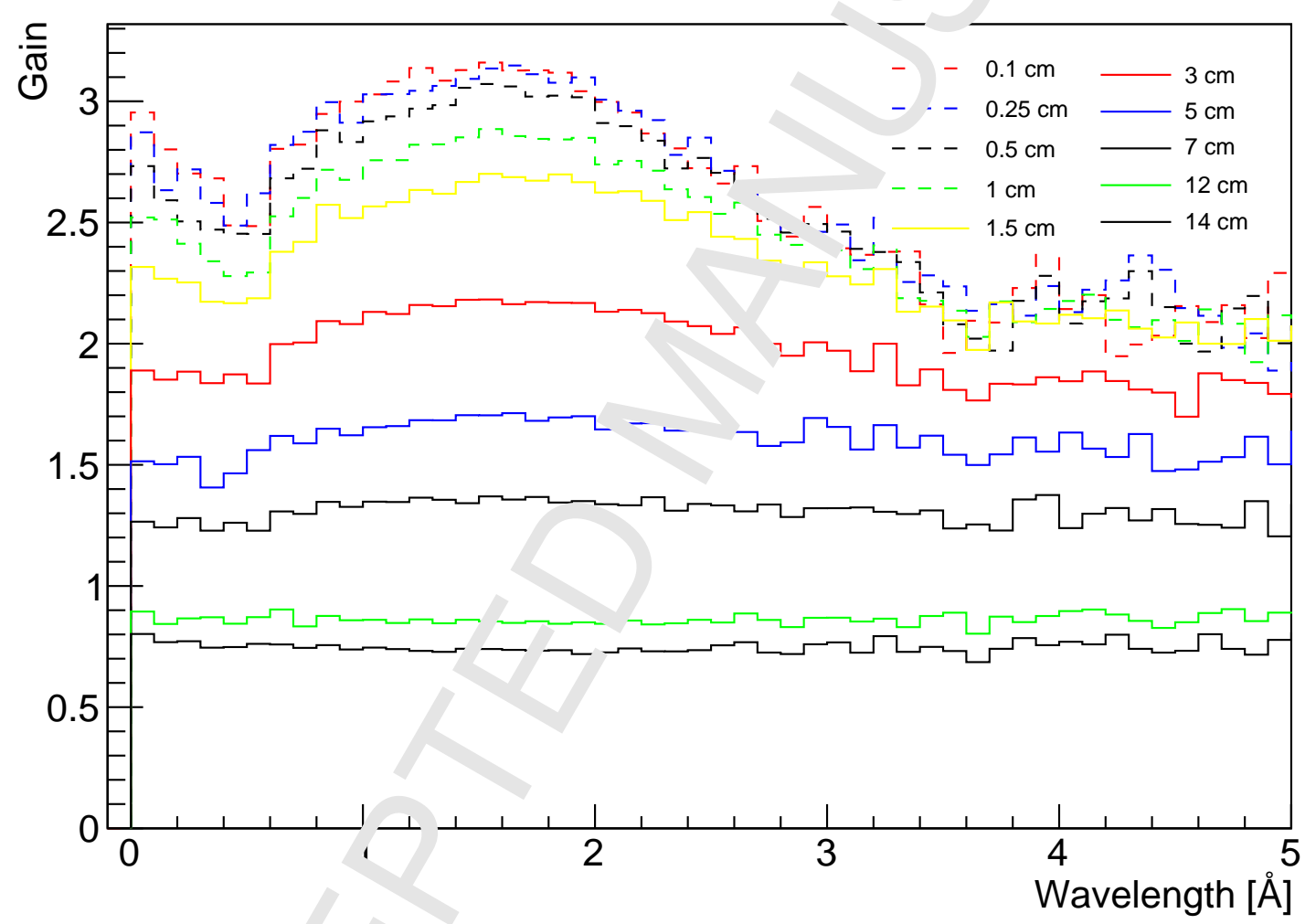




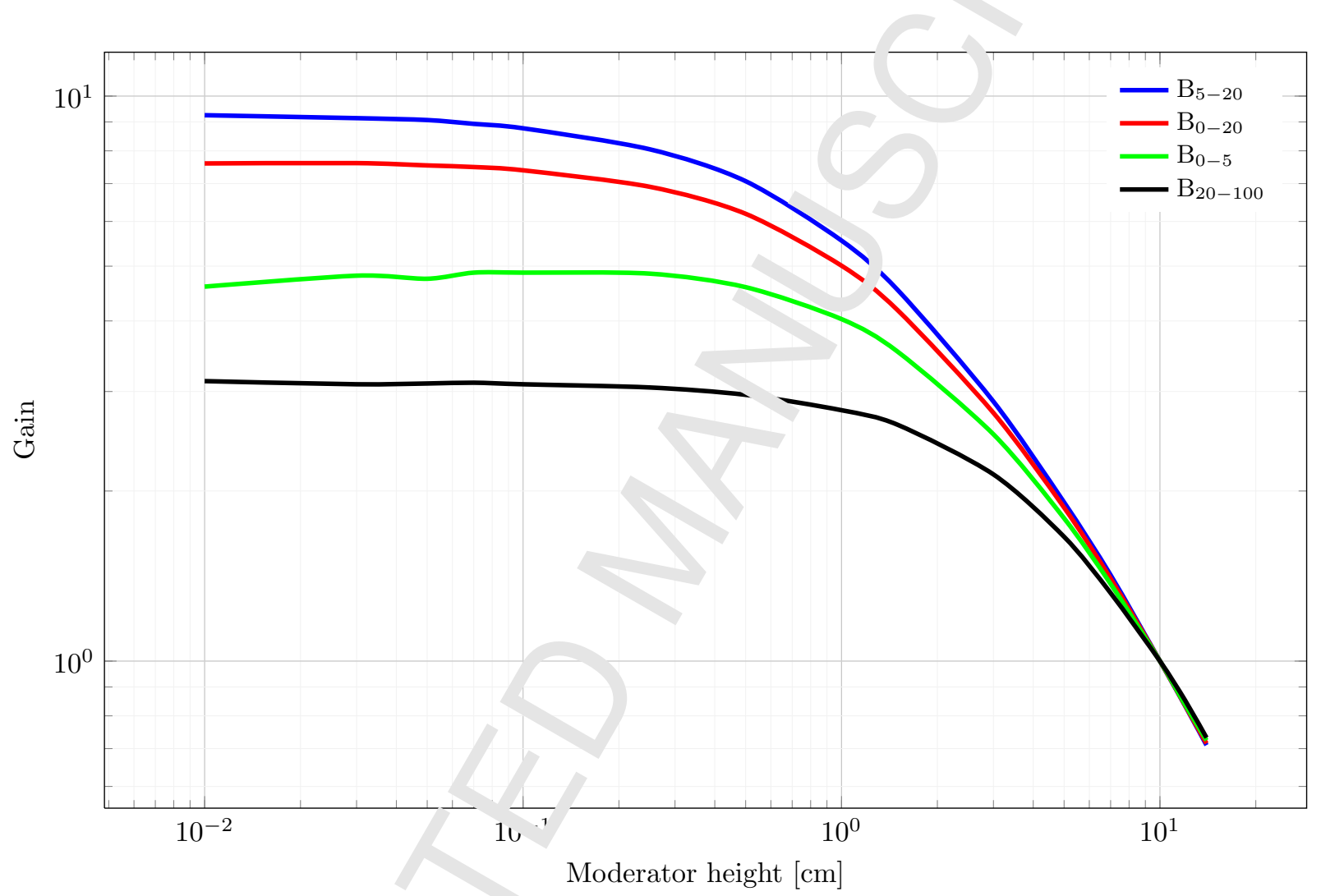

Figure 6. Cold and thermal integ ted srightness as a function of moderator height, calculated from the geometry of Figure 1. Neutrons are extracted in an xit dirt in perpendicular to the incoming proton beam, at the center of the $120^{\circ}$ sector of Figure 1. The brightness is alcu' tted 'or a 6 - $\mathrm{cm}$ wide horizontal width, the vertical range being equal to the parahydrogen height. Integral brightnesses art la $\mathrm{a}^{\dagger}$ ve to the brightnesses for the $10 \mathrm{~cm}$ high moderator. 
applications in neutron scattering (see AppendixA): for cold neutrons, $B_{0-5}$ below , meV (4 $\AA$ ), $B_{5-20}$ between $5 \mathrm{meV}$ and $20 \mathrm{meV}(2 \AA)$, averaged over the cold moderator surface. for : srmal neutrons, $B_{20-100}$, between $20 \mathrm{meV}$ and $100 \mathrm{meV}(0.9 \AA)$, averaged over the thermal mode ato surface, was used.

Figure 6 shows the relative gain in thermal and cold brightness as a function of $\mathrm{v}$. . moderator height, for integrated brightness in different energy ranges. We observe for all (thermal and colu, integrated brightnesses a general increase by reducing the moderator height.

We argue in the following that the gains observed are in part due to "merc.' nroperties of hydrogenous moderators, related to the mean free path of thermal neutrons in su h mate als. The additional gain in parahydrogen with respect to water can be attributed to the prop - jies u ius total neutron cross section. The gain is particularly strong for the cold moderator in the wi rel ngtl region between about $1 \AA$ and $3 \AA$ (Figure 4).

$B_{5-20}$ reaches a constant value for moderator height appro ${ }^{\text {hing }} \mathrm{z}$ : 0 . For colder neutrons, $B_{0-5}$ slightly decreases after reaching a maximum for about $2 \mathrm{~mm}$ height.

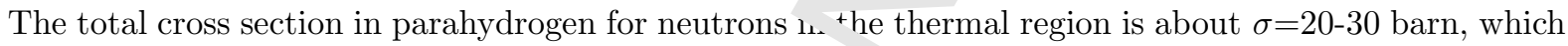
translates to a mean free path (MFP) in parahydrc af about $1 \mathrm{~cm}$. For water, the MFP of thermal neutrons is about $0.3 \mathrm{~cm}$. For the moderator height $d \mathrm{n}$. ch lower than the MFP, the number of collisions, including those leading to neutrons exiting the moder $t^{\omega_{2}}$, is proportional to $\sigma d$; as the brightness is averaged over the emission surface with area $A=W d$ ( $\iota_{\text {. }}$ 'plvulct of width $W$ and height $d$ ), one can expect that for small moderator thicknesses $B$ is independent of $d$. This explains the near constant value of $B$ observed in Figure 6 for low moderator heights.

We consider in the following subse tions 'iff rent contributions to the observed gains. We discuss first the role of the reflector.

\subsection{Reflector response}

The reflector surrounding ne ioderator has the function to return neutrons that have not gone through the moderator, or that have aped from it, to the moderator itself, giving them another chance to be moderated and redirecte . tor ards the beam extraction channels. For long pulses the time response of the reflector (of the order of $1 \mathrm{u}^{\prime}$ 's 's of no concern. Beryllium is the material of choice for reflectors in pulsed neutrons sources. T? e remo $\mathrm{zl}$ of beryllium around the moderator to extract thermal and cold neutrons has the effect of reducing $: \sim$ aghtness with respect to the maximum theoretical unperturbed brightness, i.e., the configuratic 1 where the moderator is fully surrounded by reflector material.

The amount ol * " ctor material removed increases with the height of the moderator, if neutrons are extracted from ive ntıre moderator surface. However, it also varies in the horizontal plane (where instruments are placed), dept ding on the beam extraction configuration and in particular on the number of beamlines that point to the moderator. 
We therefore consider separately these two aspects: the effect of the removal of eflector material for moderators of different height, and the effect of removal of reflector material in the $h$,rizon 1 plane of beam extraction.

In Ref. [5] we examined the unperturbed brightness. Comparing the unpertus d brightness of $10 \mathrm{~cm}$ and $3 \mathrm{~cm}$ tall moderators, it was found that the $3 \mathrm{~cm}$ tall moderator is 1.6 timas $\mathrm{br}_{5}{ }^{+} \mathrm{er}$ than the $10 \mathrm{~cm}$ tall moderator, for neutrons below $5 \mathrm{meV}$. It was also found that for a $10-\mathrm{cm}$ all .10 wator, the unperturbed brightness is a factor 1.7 higher than the perturbed brightness, calculated for a nnfiguration with two $60^{\circ}$ beam extraction openings in the reflector.

On the other hand, from Figure 6 the cold perturbed brightness " Ferteu between two moderators with height of $10 \mathrm{~cm}$ and $3 \mathrm{~cm}$ is of a factor 2.5 , which is close to the $\mathrm{vr}$ unct of the two gain factors. Thus it appears that the overal brightness increase effect is due to two me n factors, of roughly equal amount; one due to removal of reflector material, the other, observed in the nmpar son of the unperturbed configuration, due to other physics effects. It is however not possible to fully u - antangle the two effects: in the unperturbed configuration, by reducing the height of the moderator $t_{1}$ amount of beryllium is also increased; therefore the brightness increase in the unperturbed configure in is due in part to increase of reflector, in part to additional physics effects discussed below.

Besides the effect of the reflector on the different $h_{e_{c}}$ hts of the moderator, there is an effect also on the angular size of the opening for beam extraction. רstr un..ents at ESS will be arranged around the moderators covering four angular sectors of $60^{\circ}$, which will be paired forming two large angular sectors of $120^{\circ}$, one consisting of the North and West sectors the ot 1 or one of the South and East sectors (see Figure 7).

In order to further study the effec ${ }^{+}$of $t_{\perp} \cdot \mathrm{r}$ ilector removal, we considered two geometries (Figure 8), with different openings in the reflect $r$, $v$ nich were both considered for ESS: a configuration with two large opening configuration of $120^{\circ}$, wit $\_21$ be . ports per opening, and a configuration of two openings of $60^{\circ}$, with 11 beamports per opening.

Figure 9 shows the brightr ess , istributions for individual beamports. As a result of the reflector removal, going from $60^{\circ}$ to $120^{\circ}$ res'llts .. an average brightness loss of $6 \tilde{\%}$ for the $3 \mathrm{~cm}$ moderator, while it is twice as much for the $10 \mathrm{~cm} \mathrm{r}$ ode ator The effect is larger for a taller moderator, because of the larger amount of beryllium removed.

In the original de sign of i ie TDR two identical volume moderators (placed above and below the target) viewed two face-t - ' ace $u_{\mathcal{Y}}$ nnings of $60^{\circ}$ [1], so that half of the beamports of one $120^{\circ}$ sector would point to the top modera or, the ther half to the bottom. This had the advantage that each moderator would have two openinge of $6 \mathrm{v}$, and not of $120^{\circ}$, thus increasing the amount of reflector surrounding the moderator. It had howevt ne disadvantage that two different moderators had to be operated, as the use of a single moderator for the whole instrument suite would have resulted in a too big loss of brightness. Such loss would be lower in the case of low-dimensional moderators. 
A goal of ESS is to provide a beamport grid covering an angle of $240^{\circ}$. In practicf the choice, by using low-dimensional moderators, is between a single flat moderator with two $120^{\circ}$ openir gs, or 'wo identical flat moderators, placed above and below the spallation target, with two $60^{\circ}$ openings e ch. As shown in Figure 9 , the difference in brightness is of about $6 \%$. This is calculated considering a flat mo rator above the target, and a steel reflector at the bottom. In reality, if a flat moderator with two $60^{\circ}$ npen. rs is placed at the top, another moderator must be placed at the bottom. Cross-talk effects bet een op und bottom moderator exist (see Section 2.8), according to which the brightness of a moderatrm is rei red, if another moderator is placed below the target, by at least $5 \%$. Therefore, a single low-d mension al moderator with two $120^{\circ}$ openings will have in a real situation about the same performance - ¿wo « $\cdots$-dimensional moderators with two $60^{\circ}$ openings each. The performance of the single moderator ar pe $f$ rther increased if a fast neutron reflector on the other side of the target is designed to increase $1 \mathrm{w}$ nerıormance (see Section 2.8), which is not possible if there are two moderators.

In summary, we see that the effects related to the reflecu - play two roles, both in advantage of lowdimensional moderators: first, the brightness increase etı 't by reducing the moderator height; second, the possibility to use a single moderator, which would nc : - m heen acceptable for tall moderators, because of the stronger brightness decrease.

The latter finding had a profound influence on he ssign of the ESS facility, as it was understood at an early stage that only one low-dimensional is. `retaw ur was needed. By following this path, options on the use of a second moderator from the start of ESs were investigated. After the realization that all of the ESS initial instruments would perfor $\mathrm{a}$ well $\times \mathrm{v}$ pointing at the top moderator, as discussed in [13], the decision was that only one moderator noulu he installed at the start of ESS, thus adding a wide array of possibilities for future upgrades usir th pla e below the target. Such an approach also reduces costs and risks of malfunction.

\subsection{Physics effects}

\subsubsection{Parahydrogen}

Liquid parahydrogen is $\mathrm{t}^{1} \mathrm{e}$ chosen material for cold neutron production at ESS, and is used in other facilities including J-PARC. Th advantage of using pure parahydrogen, as opposed to a mixture of orthoand parahydrogen, $\mathrm{s}$ ems fro $\mathrm{a}$ the peculiarity of its total neutron cross section $[14,15]$ : below about $80 \mathrm{meV}$ the total cross sertion a decreasing, from a value of about 30 barn, until reaching about 1 barn below $15 \mathrm{meV}$. The $\mathrm{r}$ ison for this drop is due to the spin-dependence of the neutron-proton interaction, and to the spin-dependen : -...urference between the neutron wave functions scattered from the two protons in the hydrogen mor r e [16]. At $20 \mathrm{~K}, 99.8 \%$ of hydrogen is in parahydrogen form at thermal equilibrium [17]. As the main reaci on for neutrons in the meV energy range is the para- to orthohydrogen spin-flip transition, which requires $14.7 \mathrm{meV}$, this reaction is not possible below this energy, explaining the drop in the cross 
section. The inelastic cross section for para- to orthohydrogen, in the range betweer $14.7 \mathrm{meV}$ to about $70 \mathrm{meV}$, increases from about 1 barn to about 30 barn, which is comparable to t e elc tic scattering in orthohydrogen at the same energy. The density of liquid hydrogen and the beb vio $\mathrm{r}$ of its cross section are responsible for several effects relevant to moderator neutronics, namely: $t_{L_{\star}}$ thermalization of the neutrons in about $1 \mathrm{~cm}$ from the moderator walls; the near-transparency to cold neu "ons; the non-complete thermalization of the neutron spectrum.

The neutron spectrum entering the moderator has a strong thermal $\cdots$ mpu `nt, due to the presence of the premoderator on one side (between target and moderator), and of he bery ium reflector, which returns partially moderated neutrons to the moderator, on the other sid Figu- 10 shows the energy spectra calculated in regions above and below the moderator, namely the va' or p? moderator, and a cooling water layer placed in the bottom part of the reflector. Neutrons from th reflector side are mostly thermalized.

In Figure 4 a peak in gain factor is observed for neut n $_{\mathrm{S}}$ in ${ }^{t} \mathrm{le}$ wavelength range between about $1 \AA$ and $3 \AA$, corresponding to an energy range between abou ${ }^{n} \mathrm{meV}$ and $80 \mathrm{meV}$. These neutrons are not fully thermalized in the moderator, and most of them twe probably only one collision before exiting the moderator. Because of the small MFP, such neutror ... nncentrated on the edges of the moderator, on both target and reflector sides. Due to the sudder drop if the total neutron cross section in parahydrogen below $70 \mathrm{meV}$, the MFP of cold neutrons increases $\mathrm{c}$ as ' $10 \mathrm{~cm}$ : the medium becomes quasi-transparent to cold neutrons which therefore have low proba' '1luy iv be scattered.

This effect in most evident in Figure 11, which shows the vertical distribution of the brightness for neutrons in the wavelength range betwef $11.5 \mathrm{~A}$ and $2.2 \AA$ (i.e., corresponding to $B_{17-36}$, where the gain observed in Figure 4 is maximum), for $r$ oder ' $^{\prime} r$, of $1.5 \mathrm{~cm}, 3 \mathrm{~cm}$ and $10 \mathrm{~cm}$ height. The two peaks get closer to each other for flatter moderators. The fact ihat, for this energy range, the brightness is enhanced at the edges of the moderator, explains $\mathrm{t}^{\prime}$.e large $\mathrm{c}$. $\mathrm{n}$ seen in Figure 6: for the $10 \mathrm{~cm}$ tall moderator, the brightness in the central part of the modera or is s " nngly reduced, leading to a surface-average brightness decrease. The concentration of neutrons tor ard: the edges of the moderators is also evident in the brightness distribution maps of $B_{0-20}$ shown in Ficure + for moderators of height of $1.5 \mathrm{~cm}, 3 \mathrm{~cm}$ and $10 \mathrm{~cm}$. These maps are pinhole images of the brightness dist but'on on the moderator surface. For the 10-cm tall moderator there are two areas in the moderator of in " sed brightness, one on the bottom side of the moderator (the target side), one on the top part ( he refle tor side). A similar figure was calculated for the coupled parahydrogen volume moderator at J-P^ $\mathrm{RC}[\cdots \cdots$. This non-uniform brightness distribution has been experimentally confirmed by a brightness nap me isurement of the J-PARC coupled moderator [19]. Our calculations show that the effect is much less piviounced for the $3 \mathrm{~cm}$ moderator, and almost cancelled for the $1.5 \mathrm{~cm}$ one. This feature is apparent $a_{1}$, ,rom Figure 13 and Figure 14 in which the brightness $B_{0-20}$ and $B_{0-5}$ projection across the moderator he.ght, as a function of the vertical position, for three pancake geometries of $1.5 \mathrm{~cm}, 3 \mathrm{~cm}$ and $10 \mathrm{~cm}$ height, is shown. The increase of the brightness at the moderator edges is less pronounced for 
colder neutrons. We interpret this result as due to the fact that to slow down neutrons rom thermal to cold energies below $5 \mathrm{meV}$ on average requires more than one collision, which would ex end $\therefore$ volume of the production region of neutrons of this energy.

\subsubsection{Water}

The brightness increase is observed also for the water moderator, alt' oug ' 'ass pronounced than for parahydrogen: it is of about a factor of 2 (Figure 6), from a $10 \mathrm{~cm}$ moderate to a $3 \mathrm{~cm}$ moderator.

We can expect the reflector effect to be similar for water and $\mathrm{p}$ rahyd igen. The MFP of thermal neutrons in water is $3 \mathrm{~mm}$, which contributes to an increase in brightn ${ }^{*}$ for ${ }_{10}$ dimensional moderators. However, the edge effects observed for parahydrogen, due to its c oss c ct. on, cannot be observed in water, and therefore the overal gain is lower. Figure 15 shows the dist. 'hution $1 B_{20-100}$ across the height of the water moderator. As expected, an overall brightness increase is obser ed, but without edge effects.

\subsubsection{Directionality}

The moderator and reflector response, combined with ${ }^{\prime}$ properties of the parahydrogen, result in a directional emission of low-dimensional cold moderatc s: $\uparrow$ t ...oderator-shape induced directionality translates into higher flux in the horizontal direction wi. . rou hly triangular angular dependence and a FWHM of about $40^{\circ}$. See Figure 16, as well as Ref. [6]. The rhysical reason for the directional emission is related to the properties discussed above; the cold neutru. density increases near the interface of a parahydrogen moderator with the reflector within a der ${ }^{+1}$ of the order of the mean free path of the thermal neutrons in parahydrogen (about $1 \mathrm{~cm}$ ). The cc + neutre 1 emission from a parahydrogen moderator, in contrast, effectively happens from a volume $\mathrm{w}^{;}$hin the 0 -times higher mean free path of cold neutrons measured from the neutron emission window $u^{2}{ }^{+}$.e $\mathrm{m}$,derator. Thus the neutron emission probability is higher in directions where a larger volume ${ }^{\complement}$ the moderator material is seen.

In contrast and comparison the an beam from the water moder ،tor Figure 17), shows a much lower directionality. The directionality of the water moderator in the vf tical a. ’ction roughly follows the expected cosine dependence [26], displaying a broad peak falling to ze $\mathrm{z}_{2}$ ir ens' $\mathrm{y}$ at $90^{\circ}$.

\subsubsection{Neutron abso ption}

Due to the sim- 'ican wosorption cross-section of thermal neutrons $(0.3$ barn at $2200 \mathrm{~m} / \mathrm{s})$ in hydrogen, a volume mode ator tel ds to function as a parasitic absorber. This absorption term is reduced in a flat moderator, dne to un lower content of hydrogenous material. We calculated that about $10 \%$ of the neutrons entering a $10 \cdot r$. tall cylindrical moderator are captured in hydrogen, while about $3 \%$ are captured in a $3 \mathrm{~cm}$ tall moderar sr. Therefore the relative gain in using a flatter moderator is at the per cent level. Similar gains are found for water. 


\subsection{Total neutron emission and heat load}

Figure 18 illustrates two additional aspects of low-dimensional moderators, by cumpar ${ }_{\star}{ }^{*}$ total neutron emission and heat load in the cold moderator as a function of the moderator $r$ eigh . The total neutron emission or intensity is proportional to the product of the brightness and the avea os he emission surface of the moderator.

The total intensity approaches a maximum for moderator heights $\mathrm{b}$ rger than about $3 \mathrm{~cm}$ : a 3 -cm tall moderator, while it is about 2.5 times brighter than a $10 \mathrm{~cm}$ one, ...ivers . ughly $80 \%$ of its neutron intensity. The higher brightness almost compensates the smaller emissic ? surfac by providing a total number of emitted neutrons almost as high. Above $10 \mathrm{~cm}$ height, the total number of neutrons emitted approaches a constant value. The implication of this result is that to increa - we $\mathrm{j}$ itensity of neutron emission one should not increase the height of a parahydrogen moderator. Ratı - other types of moderators should be considered, such as large-volume $D_{2}$ moderators.

The heat load in the moderator (parahydrogen and aluminu - cryogenic parts) increases with the moderator volume (black curve in Figure 18). Thus, flat modeı ' ${ }^{\text {ors }}$ have an advantage in terms of requirements on the total capacity of the cryogenic systems. It $m_{\llcorner}$it $\ldots$ id however that there are other challenges in

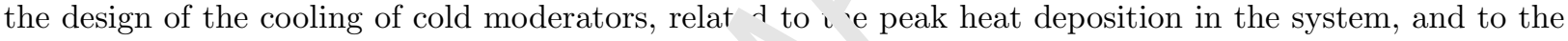
flow rate of the hydrogen, that must be addressed in the ngineering design.

\subsection{Premoderator}

The premoderator changes the shape $\mathrm{c}$ the ne tron spectrum from the spallation target, bringing neutron energies down from the $\mathrm{MeV}$ to the $\mathrm{t} \mathrm{rrma}_{2}$ " 9 ge. Usually the premoderator is a layer of water between cold moderator and target. Its thir nes is retermined by a balance of optimal spectral shaping, by the need to minimize the distance tar ,et-mou ator, and to minimize neutron absorption.

A thickness of $3 \mathrm{~cm}$ was found an nptimal; concerning the lateral dimensions, we found that a diskshaped, extended premodera or, ith radius practically equal to the Be reflector, gives a substantial gain in both thermal and cold hrign. ess. Figure 19 shows the relative variation of cold and thermal moderator brightness with the rad' is $\mathrm{C}$ a disk-shaped premoderator. In this sensitivity study, the gain is different depending on the matarial $p_{2} \sim d$ outside the premoderator, i.e., in the ring with inner radius equal to the radius of the premor erator, nd outer radius equal to the radius of the beryllium reflector. If there is void, there is a steady : srean of both thermal and cold brightness, reaching the maximum for a $30 \mathrm{~cm}$ radius disk. By placin a reflec or material (in this case, beryllium with $30 \%$ water cooling), there is a maximum cold brightness for a premoderator of about $15 \mathrm{~cm}$ radius. This could be an option for future upgrades. The present config ${ }^{\prime \prime}$, ion has a steel ring with inner radius of $30 \mathrm{~cm}$. 
Figure 7. View of the 42 ESS beamport inserts each of $3.5 \mathrm{~m}$ length, located in four beam vtre tion sectors of $60^{\circ}$. The proton beam comes from the right in the figure. The tips of the beamports are at $2 \mathrm{~m}$ from cen - of the moderator. The beamports are labeled according to the name of the sectors (North, South, East, West). ular $s_{\mathrm{s}}$ cing between beamports in the North and East sectors is of $6^{\circ}$. In the West and South sectors alternating angul $:$ spa $\mathrm{ng} .5 .7^{\circ}$ and $6.3^{\circ}$ between the beamports is used.
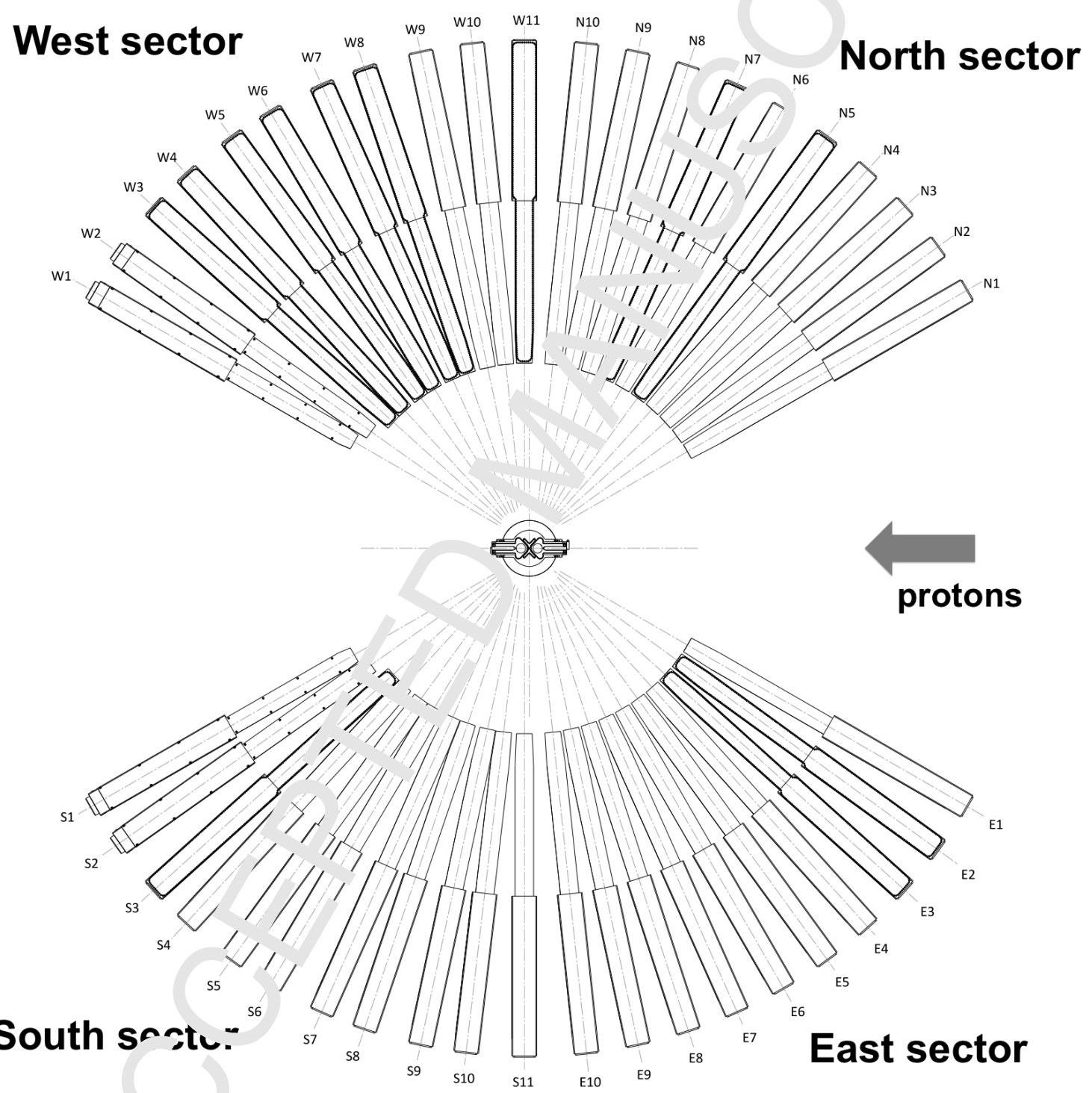


\subsection{Orthohydrogen contamination}

Figure 20 shows the variation of the cold brightness from a 3-cm tall moderator (in this ce ${ }^{\circ}$ of butterfly-2 type, see Section 3.3) as a function of the parahydrogen fraction in the moderatr . N iximum performance of low-dimensional moderators requires pure parahydrogen and ideally $100 \%$ parahy 'rogen should be used. The aim during ESS operation is to achieve a parahydrogen fraction of at le - 99.57 by using catalyzers. Thus small fractions of orthohydrogen are expected, which may slightly af sct + . 1 neutronic performance of the moderator. In our calculations we assumed $99.5 \%$ parahydrogen, $r_{\text {n. w w w }}$ ? to specify it as $100 \%$ in the model; the reason is because recent experimental results [15] indic te that the ENDF/B-VII measured cross section did not account for an impurity of orthohydrogen of $r . v \%$ in tne hydrogen samples.

In the following two subsections we discuss two properties $\mathrm{w}$. $\mathrm{Al}$ ar not specific to low-dimensional moderators, but are linked to the design of the moderator systc 's. The information on the size and location of hotspot of neutron production indirectly dictates ?e dir ension of the moderators, as well as the ideal location of the cold and thermal neutron moderators. The cross-talk between the areas above and below the target is also of interest and can be possibly ex r ' ited to increase the moderator brightness.

\subsection{Hotspot}

Figure 21 shows the map of high-energy $\left(E>0.1 \mathrm{Y} / t^{-\top}\right)$ neutrons from the spallation-evaporation process in the tungsten, assuming $2 \mathrm{GeV}$ protons. The nlan is the color map is at the vertical coordinate $Z=8$ $\mathrm{cm}$, just above the tungsten target and below the water premoderator. To give an idea of the relative dimensions of the moderators and of th neutre a hot spot, the geometries of the butterfly (discussed in detail in Section 6) and pancake mod rator ar, superimposed to the neutron flux map, even though the moderators are placed above the $\mathrm{p}^{\prime}$, ne wher : this fast neutron map is calculated. The dimensions and lateral positions of both moderat , $r s$ in $\iota_{\text {. }}$ figure are optimized for maximum cold brightness across the $120^{\circ}$ sectors. This figure confirr.s tha 'he dimension of the moderators should be comparable to the source of spallation-evaporation nev ron, as expected. This figure also suggests that it is convenient, if possible, to place both thermal and cols sources above the hot spot. This is one of the arguments in favor of the butterfly moderator ( $\mathrm{sec}^{\mathrm{Sec}} \mathrm{Sen}^{5}$ ) and the thermal moderator brightness is higher if the water moderator is placed closer to the $n$ nteı $f$,he hot spot.

\subsection{Cross talk betwe - mor zrators and fast neutron reflectors}

The beam e tractio system at ESS is designed such that neutrons can be extracted from two angular sectors of $120^{\circ}$ eac. . $.0 m$ above and below the target. The two beam extraction areas above and below the target art $\eta \mathrm{c}$, completely independent of each other as neutrons generated or reflected in one region of the target can tr. vel to the other side if their energy is high enough to cross the target wheel. The size of this effect might be increased by the lack of moderating material in the target, which would increase the 
absorption of neutrons in the target itself. Thus, the brightness of the moderator placer' above the spallation target may depend to some extent on what is placed on the other side.

At ESS this opportunity can be exploited, considering also that ESS is a long $\mathrm{f}$ ılse acility, and therefore an increase of the width of the time distribution of the neutrons within the lons. 1lse is not of primary concern. If only one moderator system on the top side of the target is present it is vorthwhile considering different options to design a fast neutron reflector placed below the target to ir crewe the brightness of the

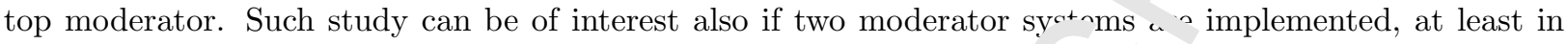
some cases, for instance if the second moderator system requires a sigr ficant $\mathrm{x}$ 'utron and gamma shielding to allow reaching very low temperature (this is the case for some mor' - atoı w Ultra Cold Neutrons or Very Cold Neutrons) [20-22]. In that case, the presence of these shield $r$ sich are typically heavy metals (such as $\mathrm{Bi}$ or $\mathrm{Pb}$ ) with good properties of fast neutron reflection, coula ' exploited to increase the brightness of the top moderator.

We considered a few cases using some typical fast neutru reflector materials. Table 1 indicates the gain factors relative to the two-moderator configuration, e., a configuration where a moderator/reflector system similar to the top one is placed at the botto: . mhic is the configuration that results in the lowest brightness, due to the presence of the extended pramode ator, as well as the water moderator and coolant, which thermalize the fast neutrons preventing them ¿ ols. rossing the spallation target and reaching the top moderator. On the other hand, if materials that an ruiect fast neutrons, such as copper, lead, tungsten, or steel, are used, a significant brightness increase is obtaned on the moderators placed at the top. If beryllium is used at the bottom, the brightness inc ease is ot as large as for heavier metals. This is due to the fact that in beryllium neutrons are reflected but a. $n$, artially slowed down, and a significant part of the neutrons reflected will be absorbed by the tur sste 1 tar jet.

The effect depends on the mat rial usti but also on the amount of water coolant used. The calculations with a tungsten reflector are purformı ${ }^{\top}$ using the same effective density of the tungsten in the spallation target, and assuming helium cool ig. It is worth noting that the reflectors absorb a heat load of the order of $500 \mathrm{~kW}$ (calculated for steer - fflector). Their cooling is therefore quite demanding.

\subsection{Background from hig, 'ner y neutrons}

Neutron scatteriı g resea $\mathrm{h}$ mainly uses slow neutron beams with energies below a few eV. Fast neutrons can preferentially eccap th target shielding through the slow neutron beamports. They are also emitted by scattering on th struct es around the moderators also viewed by the beam lines. High-energy neutrons can be thermalized by $\ldots$ - ' ering on structures and materials in the experimental halls and ultimately contribute to the backgi ur $d$ of the slow neutron detectors in the neutron scattering instruments. This is a an issue for all neutron so rces; we want to investigate if in the low-dimensional moderator concept adopted for ESS the slow neutron signal to fast neutron background emission ratio will be more or less favorable than for 
existing spallation sources.

\subsubsection{Figure of merit}

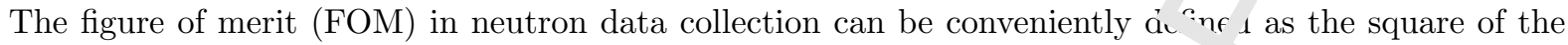
signal-to-noise ratio (where by definition both signal and noise are expressed in uı. ` of counting time). This corresponds to the data collection rate, i.e. the inverse of the time needed $+\mathrm{s}$ ac $\ldots$ re a given signal-to-noise ratio. The noise must be defined as the uncertainty of the measured value oı he signal $S$.

There are two main sources of uncertainties: the statistical preci on of he counts measured and the systematic uncertainties and instabilities, which are independent of cu ntir statistics. Considering the issues we are concerned with here, we can restrict the analysis to ne statistical precision of the data (assumed to be stable and understood concerning their origin). Oth systematic errors are independent of the neutron beam intensities, but of course they becom^ more a d more dominant, as the statistical accuracy of the data improves, e.g. by the new level of neuı $` \eta$ inuusisities ESS will offer.

The statistical noise of the signal $S$ will be proport; wi u $\vee \overline{S+B}$, where $B$ is the background. The measured raw neutron counts for both $S$ and $B$ are nropors. nal to the data collection time $t$, thus the data collection rate is the inverse of the time needed to $i r$.ieve a given statistical signal-to-noise ratio. The background $B$ has two main components: $B_{S}$ com. ny ${ }^{\text {frol. }}$ the slow neutrons delivered to the sample and its environment for the intended observations ...... coming through the beamline or through the shieldnı。 of other beamlines, etc. Thus

$$
\neg \cap M=\frac{S^{2}}{S+B_{S}+B_{F}}
$$

In the denominator the first two err s ar proportional to the slow neutron beam intensity, the last to the fast neutron production.

Shielding measures that reduce $B_{F}$-ithout any effect on $S$ are always useful, to varying degrees. If such a measure impacts both $S$ ar $\perp B$ the balance is what matters. For example, if both the slow neutron and the fast neutron production cha re by the same factor $n$, the change of FOM will be proportional to $n$. So the data collection gain of I.SS r mpared to other facilities will be proportional to the delivered neutron beam intensities (i.e. c ne I 00 times higher than the other sources) under the assumption that the fast neutron background also inc eases in the same proportions. If the fast neutron background increases less than proportiona" ${ }^{1}$ to un slow neutron production rate, the FOM will be for some experiments higher than proportional to he beas । intensity gain, primarily in cases where $B_{F}$ is comparable to or larger than $S$.

\subsubsection{Fast $r$ utr, $n$ vackground at ESS.}

The total nu. ber of fast neutrons emitted is, as for all neutron sources, about the same as that of the moderated neutrons, and it is the beam extraction and shielding equipment that ensures that only a very 
small fraction of these neutrons turn up as counts in the detectors (which have in ar dition low detection efficiencies for fast neutrons).

Figure 22 shows the simulated vertical distribution of the points of emission c ne trons with $E>1 \mathrm{eV}$ from the moderator area, for three different pancake moderator heights.

These "emission points" are defined by the backward extrapolation of the neuı $n$ trajectories entering the beam line openings to their crossing of the nominal neutron emmiting surf s e the cold moderator or to its vertical continuation. In reality, these neutrons are directly genern+ed $e_{2} \cdot e r$ by collision with atoms in the volume of the moderating material or by collision with atoms in the stru tures around the moderator such as the moderator vessel, its insulating housing, the reflector $a^{\text {r. }}$ ' the uvulants.

Since the back trajectory simply follows the direction of the $11^{4}$. on is determined at radius $2 \mathrm{~m}$, the method implicitly assumes that no scattering along the trajectory c curs. for neutrons originating elsewhere than from the moderators, this assumption is not strictly va.. " caus' $\mathrm{g}$ a "blur" to the apparent emission point of these neutrons.

The main conclusions from the figure, and considerin $_{\diamond}$ also Figure 14, can be summarized as follows:

- The total fast neutron emission by flat moderatc $\mathrm{s}$ ic less than for the tall one (about $50 \%$ for $1.5 \mathrm{~cm}$ moderator height and about $67 \%$ for $3 \mathrm{~cm} 1$. v tera $r$ height).

- There is some fast neutron emission outs. " un - oderator window, which amounts to about $36 \%$ of the total fast neutron emission for the $10 \mathrm{~cm}$ mouerator, $45 \%$ of the total for the $3 \mathrm{~cm}$ high moderator, and $51 \%$ of the total for the $1.5 \mathrm{cr}$ high , oderator.

- The ratio of the deliverable cold neutron itensity (which scales with the brightness) to the total fast neutron emission is about 3 tiı n norf favorable for the $1.5 \mathrm{~cm}$ moderator than for the $10 \mathrm{~cm}$ one.

Based on the results in Figu $\lrcorner 2 \iota$, Figure 23 shows the relative evolution of the ratio $S / B_{F}$ of the signal (which is proportional to the or neutron brightness $B_{0-5}$, cf. Figure 14) to the emission of $>1 \mathrm{eV}$ fast background neutrons that art - ning from the central $8 \mathrm{~cm}$ high effective neutron emission area around the middle of the moderator $\mathrm{Tr}$ s means that the assumed background emission conservatively also includes fast neutrons coming from ${ }^{+}+\mathrm{si}$ ' e the nominal moderator emission surface for moderators of $<8 \mathrm{~cm}$ height, while fully excludin

Our MCNPX simu' neutron moder: tors an ' their immediate vicinity emit towards the beam-line openings in the monolith shielding about as ..... $y>1 \mathrm{eV}$ energy "fast" neutrons (which are assumed to contribute to the background detected at $t_{1}$ ? $i$ struments) as slow neutrons for use in neutron scattering experiments on the instruments (cfr Figure 14 a. d Figure 22). The emission of fast neutrons geometrically peaks at the edges of the moderators, including areas around and outside the viewed nominal face of the moderator. The ratio of 
Figure 8. Pancake geometry, with openings in the reflector of $120^{\circ}$ (left), and $60^{\circ}$ right).
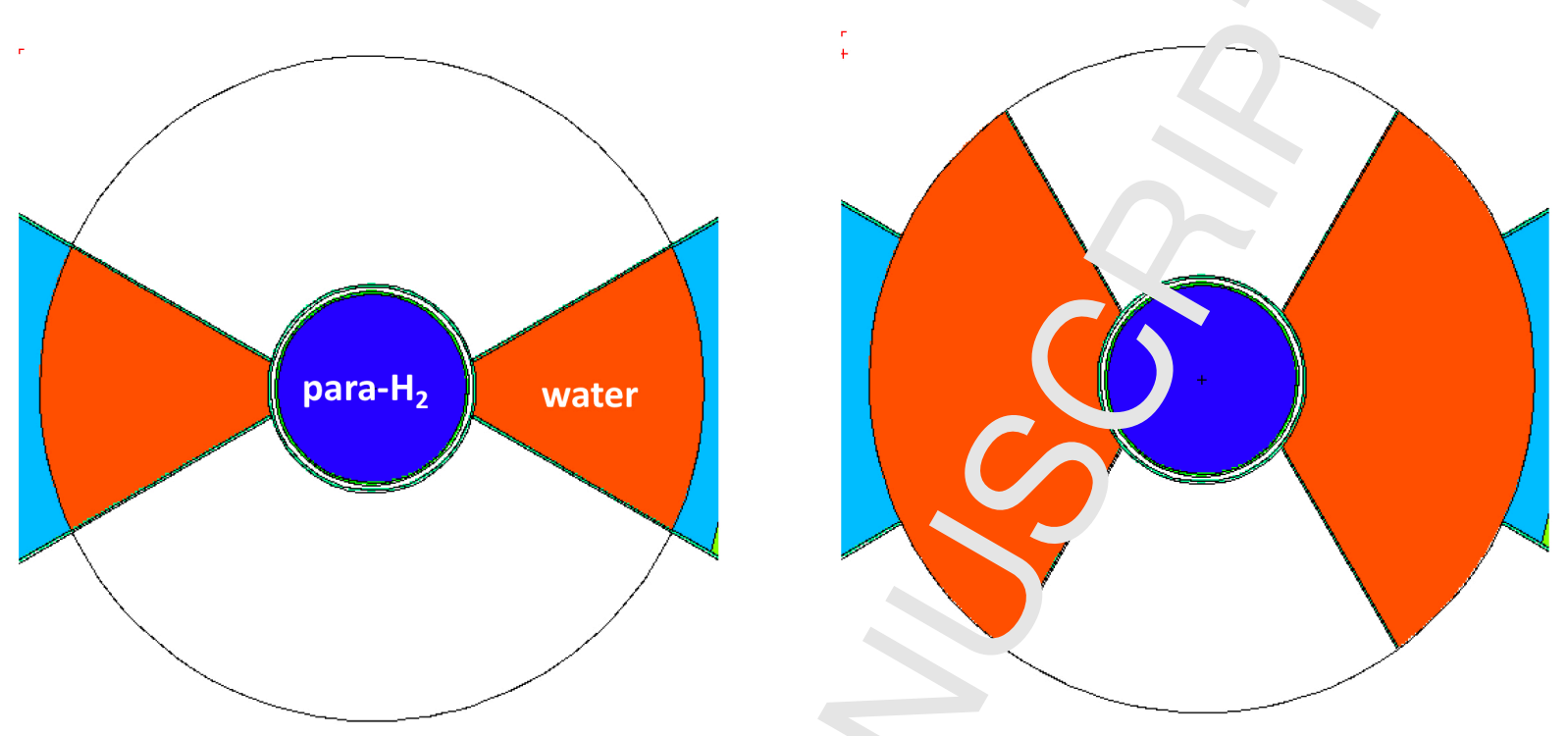

slow to fast neutron emission is significantly more fa rorajle for the low dimensional, flat ESS moderators than for the conventional larger beam cross sectl moderators at existing spallation sources.

\section{Moderator concepts}

In this section we describe the $r$ ode ator concepts that were considered for ESS. We start from the volume-moderator configuration $\mathrm{c}$ the T⿱ $\mathrm{T}\{$, then two low-dimensional moderator concepts which were seriously considered for the facil oy a a described.

\subsection{Volume moderator: $T \Gamma$;}

The TDR design was ase- on whe J-PARC coupled volume parahydrogen moderator [18]. In the TDR baseline configuration, the - are wo volume moderators filled with pure parahydrogen. The MCNPX model shown in Figure 24 eprod ces the engineering design developed during the target station design update phase [1]. The moden ${ }^{+}$ors $\mathrm{h}$ `ve a diameter of $16 \mathrm{~cm}$ and a height of $13 \mathrm{~cm}$. The moderators are surrounded by light water $\mathrm{p}$ emode ators (except for the cold neutron extraction window), of which the most important part, from the nt troni, point of view, is the layer between target and moderator, which is $2 \mathrm{~cm}$ thick. The window surte $\mathrm{o} \cdots$ cold moderators for beam extraction is of $12 \times 12 \mathrm{~cm}^{2}$. A variation from the J-PARC design is the prt ence, on the sides of the cold moderators, of thermal moderators for thermal or bispectral extraction. They consist of $4 \mathrm{~cm}$ thick water slabs with a surface area for neutron extraction of $12 \times 11$ 
Figure 9. $B_{0-20}$ angular distribution for a $3 \mathrm{~cm}$ and $10 \mathrm{~cm}$ tall pancakes, with $60^{\circ}$ ind $12 \mathrm{C}$ openings in the reflector allowing for 21 and 11 beamports with $6^{\circ}$ separation, respectively. The brightness is avera s over he extraction surface, with fixed width of $6 \mathrm{~cm}$, and height equal to the height of the moderator. Beamport ar, ,es are denned with respect to the direction of the proton beam, from left to right in Figure 8.

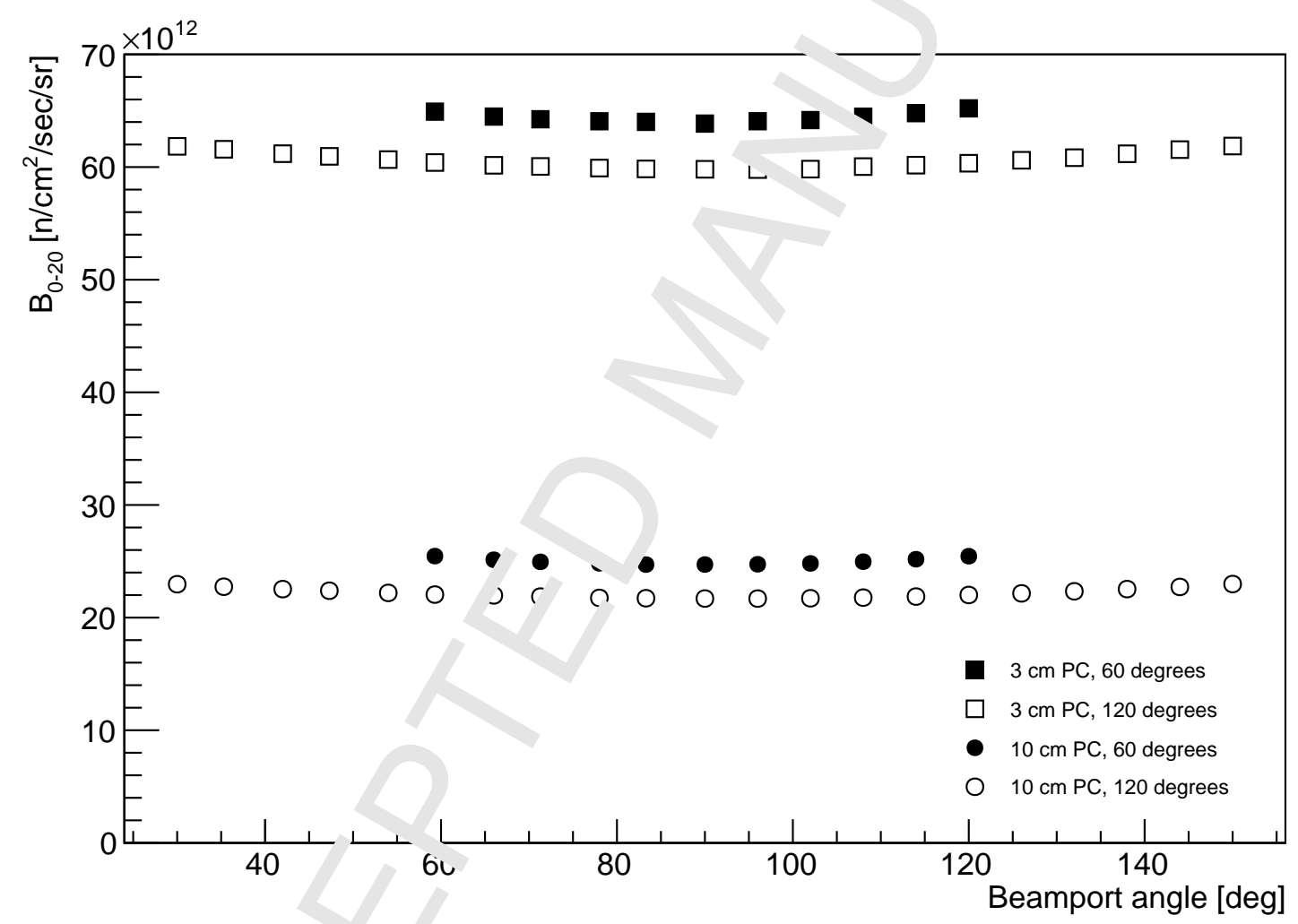


Figure 10. Average flux per unit lethargy inside the cold moderator, the water pr noderat $r$, and in the bottom part of the reflector, above the moderator. The flux is calculated using the $\mathrm{f} 4$ track-lengtl esiimator.

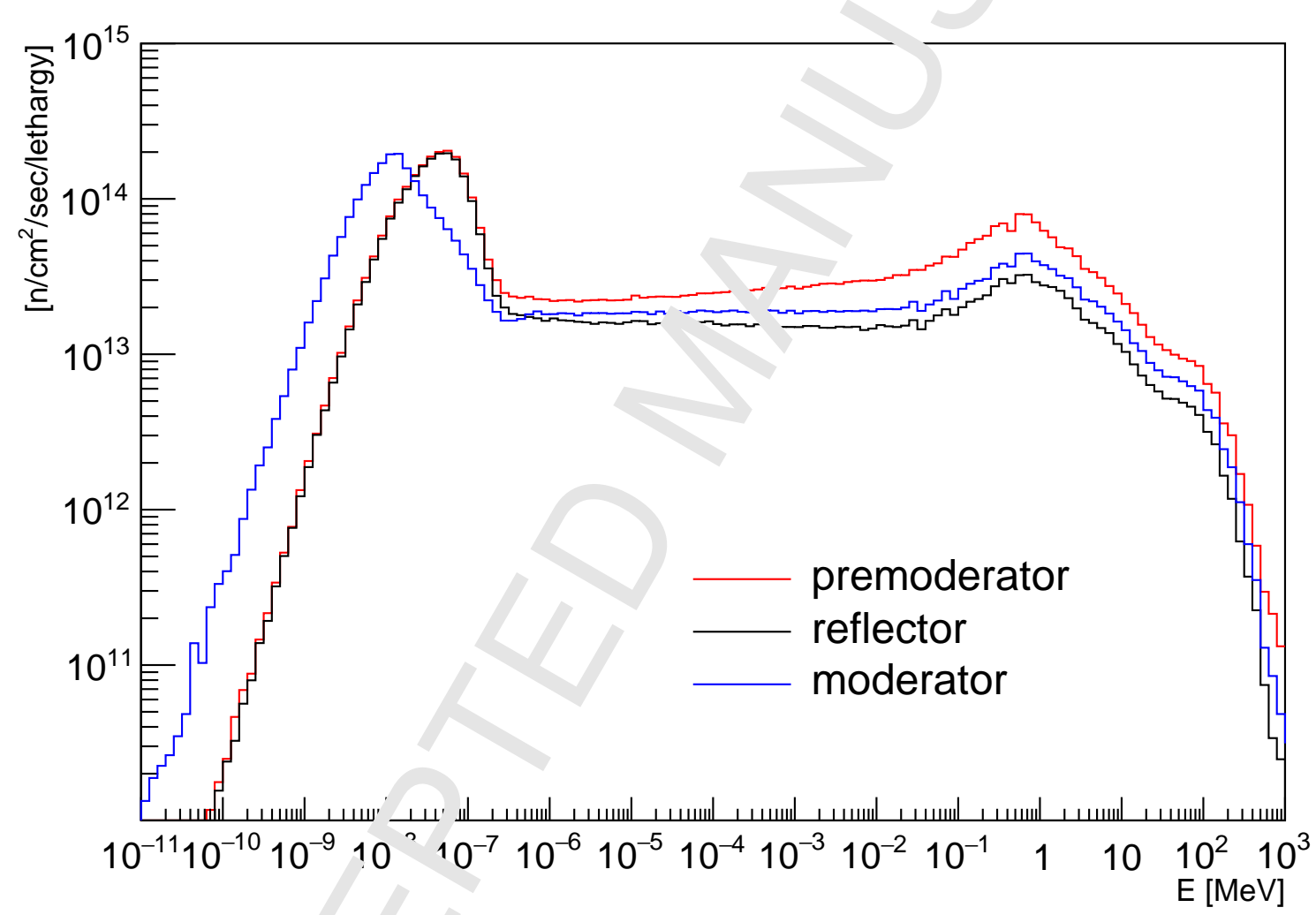


$\mathrm{cm}^{2}$. The openings in the reflector for beam extraction are of $60^{\circ}$, with two openin , s per moderator, as shown in the figure. There are two identical thermal-cold moderators, one above an $\_$ont 'olow the target. The openings in the reflector are arranged so that, of the four $60^{\circ}$ sectors, each $\left.t / O t\right)^{\circ}$ sectors, above and below the target, form a $120^{\circ}$ sector available for the beamports. More informatio. s available in $[1,3]$.

\subsection{Pancake low-dimensional moderators}

The first low-dimensional design considered was the pancake moderator $\lfloor\iota$; which has been extensively discussed in the previous section, as it forms a convenient basis to deterr ine the roperties of low-dimensional moderators. The pancake consists of a disk-shaped $(3 \mathrm{~cm}$ height. $90 \mathrm{c}$ ameter) cold moderator (see Figure 1). The basic cylindrical shape comes from the need to ser e cr a n utron instruments in a $2 \times 120^{\circ}$ suite. Thermal neutrons are extracted from the sides of the cola node_...or which contain water. The need of bispectral extraction implies that a design solution must l found o allow for bispectral extraction. For the pancake, two options are possible, and are discussed in $\iota_{\text {. }}$, nexu section.

\subsection{Butterfly low-dimensional moderators}

Butterfly-shaped moderators [23-25] consist of a 1 or ified version of the pancake, where the thermal source is moved to the center of the moderator. $\mathrm{I}_{1} \mathrm{c}_{\mathrm{c}} \mathrm{nal} \mathrm{f}$ this design is to adapt to the beam extraction configuration of ESS and provide bright bispec ${ }^{-1}$ neu ron beams for the whole instrument suite. The first idea behind this concept is to have both thermal and ld sources close to the hot spot of neutron production (see Section 2.7 and Figure 21, left), thus ^eac ing high thermal and cold brightness, and having a design capable of delivering a good bispectral bu $m$ ovf : the full $2 \times 120^{\circ}$ beam extraction sector. Two variants of this design were considered. The firs ${ }^{+}$moderator, that will be used at the start of operation, is the so-called butterfly 2 (Figure 25, bo ${ }^{\star} \mathrm{on}_{\llcorner}$, It onsists of two distinct cold moderators separated by a thermal cross-shaped moderator and will ox nerated until the start of the user program. The second variant is the butterfly 1 (Figure 25, top).

The drivers for the butte. ${ }^{q} \mathrm{v}$, esign were the following:

- place cold and the mal mod rators on the hot spot, in order to provide high thermal and cold brightness for a requi $\ldots$ extı cion area of at least 3 (height) $\times 6$ (width) $\mathrm{cm}^{2}$ for both thermal and cold moderators.

- exploit to some t tent, also the concept of tube moderators (discussed in [6] and in the appendix), thanks to t. a ger netry of the cold moderator.

- keep a 1 I r vively compact shape to ease the beam extraction: for all the 42 beamports, the thermal and cold exuraction surfaces lie next to each other, being placed on the two sides of the focal points (see Section 4.4), allowing instruments to see the brightest part of thermal and cold moderators. Such 
moderators fit well for a beam extraction in the two $120^{\circ}$ sectors; the brightnes variation across the sectors is within $15 \%$.

Before comparing the neutronic performance of the different moderator cons sts we describe in detail the beam extraction principles and implementation for ESS, to better understa. ${ }^{d}$ the ${ }_{-}$role in the design of the moderators.

\section{Beam extraction}

\subsection{Design features for instrument performance}

For a high-performance instrument suite, the moderator as: $\mathrm{m}^{\mathrm{k}} \mathrm{y}^{\circ} \mathrm{c}^{\circ}$ any facility for neutron beam instruments needs to be designed with three key performance in " atc. in mind:

1. high source brightness;

2. ability to extract the right amount of phase space f n... eamline;

3. ability to install the required number of beamli

At ESS, two additional factors were included in t? desig ' considerations:

4. ability of each beamline to freely choose bispectral mirror assembly, a bispectral source,

5. upgradeability.

Achieving high brightness, the fir $i$ perfor _. ance indicator, gave the main figure of merit used in the moderator design and is the main fu " of t ie present paper. However, the other performance indicators were also used in the moderator 'sign. In this section key features of the other performance factors are described, and in particular their link to the design of the ESS moderator assembly. All these performance indicators gave practical cor trai ts and guides on the design of the moderator. This is however only one side of the work, as ever propu d design implied an extensive study of the brightness transfer to the instruments, which in $t_{1} \cdot \eta$, ave additional inputs to the moderator design, in an iterative process. This iterative work, from sne poin of view of beam extraction and brightness transfer to the instruments, is described in detail in. [13].

\subsection{Phase spac}

Most neitron beaul instruments use the neutron beam to illuminate a sample of a material to be studied. The huge rang $r_{\perp}$ types and geometries of samples reflects the diversity of the science which can be addressed with beams of sluw neutrons [1]. The main limiting features to the required phase space of the neutron beam are, however, fairly well-known. 
Figure 11. $B_{17-36}$ distribution across the moderator height, for cold neutrons, for three cylidrical $\mathrm{r}$ derators with $1.5 \mathrm{~cm}, 3$ $\mathrm{cm}$ and $10 \mathrm{~cm}$ height, for neutrons between $1.5 \AA$ and $2.2 \AA$. Moderator edges are marked by dasheu vertica. 'ines. The vertical position is relative to the center of the spallation target.

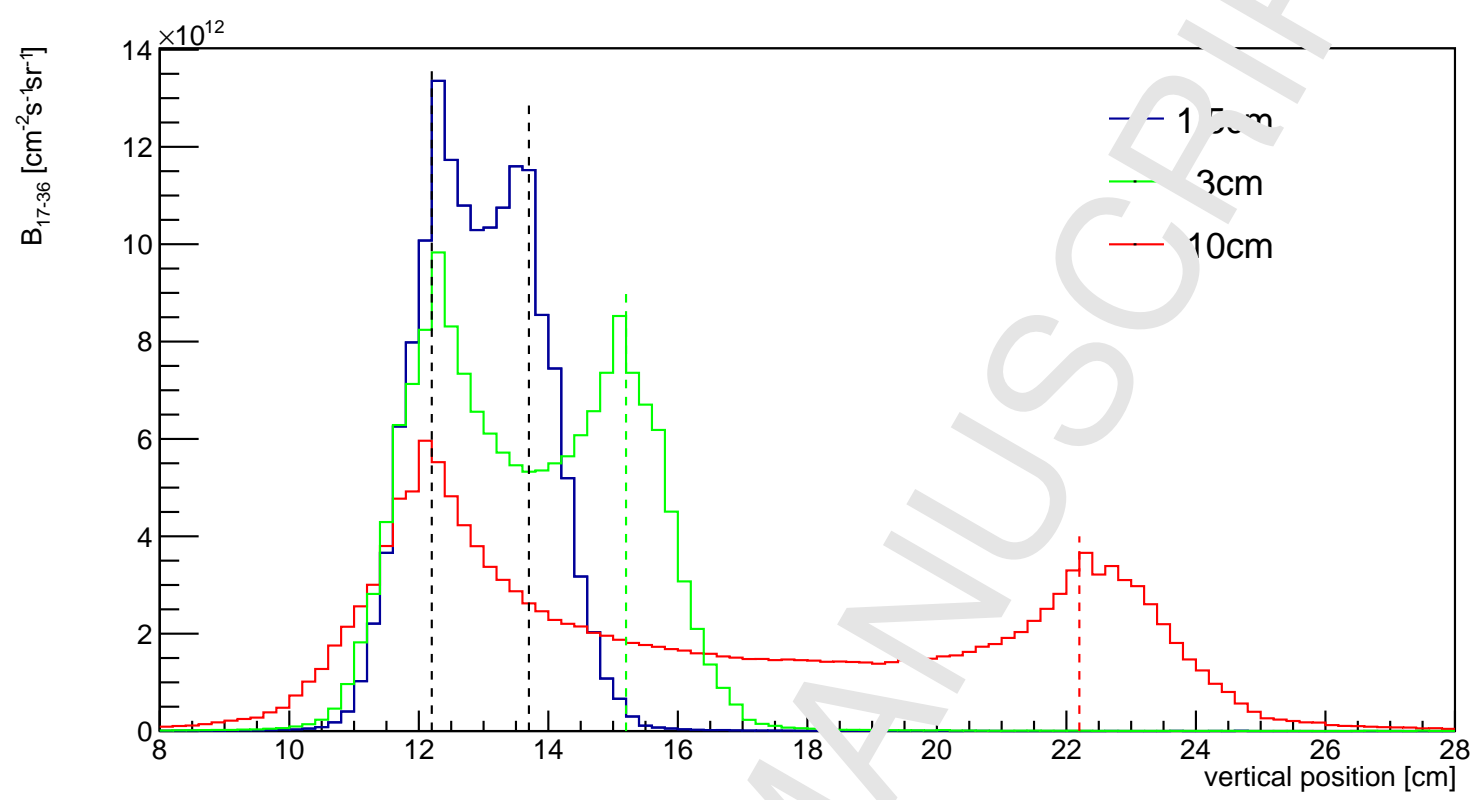

\subsubsection{Beam size}

The beam size typically needs to be rapted to the sample size. In a few cases, samples can be very large, up to several metres in linear $r$ me' sion. However, in these cases, the purpose of the neutron beam investigation is always to measure ' $\mathrm{\rho c}$. 'rof rties with a spatial resolution which is much smaller than the size of the sample, typically of +. order of mm's or less. Most neutron scattering experiments are fluxlimited, due to the intrinsicall ${ }^{-1}$ nw neutron source brightness compared to, e.g. synchrotron light sources. For optimal experimental c ' dit' ons, sample volumes for neutron experiments are therefore usually fairly large to compensate for t e lnw watron flux. For materials characterization, the sample size is typically limited by the amount results in an upper '.mit on miost samples of the order of a few cubic cm's. In many cases, researchers wish to measure muc's smal'sr sample volumes. There can be many reasons for this, ranging from sample availability and ost tc the need to measure local properties in intrinsically inhomogeneous materials. The lower limit to th samr a volume is almost always determined by the available neutron flux. Typical beam sizes are the Ilu1. the range of a few mms to a few cms. 


\subsubsection{Beam divergence}

Most neutron experiments are aimed at a measurement of the dynamic structure iactoı $₹(\mathbf{Q}, \omega)$ straightforwardly obtainable from the neutron scattering cross-section, or one of its deri ed , uantities such as the static structure factor or reflectivity profile. The dynamic structure factor contains ¿ a essential information on the spatial correlations between atoms (as a function of of $\hbar \mathbf{Q}$, the moment um . ansfer) and their time correlations (as a function $\hbar \omega$, the energy transfer). The spatial resolutio wh' ${ }^{\circ}$ h cun be achieved depends

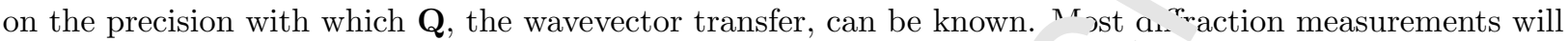
aim to achieve a $\mathbf{Q}$-resolution of the order of $10^{-3}$ or better in $\Delta \mathbf{Q} / \mathbf{(}$ '. This , irectly results in divergence resolution of $\Delta \theta / \theta$ of $10^{-3}$ or better, i.e. a beam divergence of is orus of $0.1^{\circ}$. Small-angle neutron scattering can generally tolerate a significantly more relaxed Q-re ㄱ- won but the need to measure at very low scattering angles near the incident beam, usually also require $~$ divergence of the order of $0.1^{\circ}$. Spectroscopic measurements will usually accept a relaxation of $t_{\iota} \quad Q$-res lution in order to gain flux. Todays neutron spectrometers (i.e. instruments for spectroscopic mea "rements) will typically accept a beam divergence of the order of $1^{\circ}$, and some even $4-5^{\circ}$, in order $n$ achieve the flux needed to observe the desired inelastic signal.

A typical instrument will thus need a phase space rolume of the order of $10(\mathrm{~cm}-\mathrm{deg})^{2}$ with a few instruments requiring much less and a few requiring ip . 20 times as much. For each instrument, a phase space of beam area and divergence at the samp can se specified which the neutron source should fill as completely as possible, in order to maximize flux. Each instrument needs to be equipped with a neutron optical system designed to transport this phase : sace from the source to the sample, while minimizing the loss of phase space density, in accordar e wiı. ' ' Juville's theorem (see AppendixA). Such an optical system usually consists of neutron guides ir whi $\mathrm{n}$ th : amount of phase space which can be transported is roughly proportional to the neutron wave ${ }^{1}$ ngth sy - sred, due to the reflection properties of the inner guide surface. As a result, the phase space voiume $a^{\text {' }}$ 'vered to the sample is usually strongly wavelength-dependent and often only reaches the desirer val e for longer wavelengths.

In terms of the modera ${ }^{+}$or $a_{c}$ : on, these considerations result in a requirement for the minimum viewable surface area of the mod ratc. H wever, the amount of phase which can be extracted from the moderator and the heat load and - diat $\cdots$ damage incident on the beam optics decrease with the inverse square of the distance from the $\mathrm{m}$ derator face to the entrance window of the neutron optical system. The compromise made at the ESC - to suart the guide optics at a distance of $2 \mathrm{~m}$ from the moderator center. In order to optimally de ver the typically needed phase space volume of $10(\mathrm{~cm}-\mathrm{deg})^{2}$ at this distance, the needed viewable modorator surface can be calculated from simple geometrical considerations to be about $20 \mathrm{~cm}^{2}$ in area, slight.- ess for thermal neutrons, slightly more for cold neutrons. More information can be found in $[13]$. 
Of the various moderator geometries described here, the TDR geometry provides the largest viewable

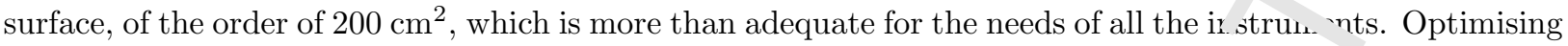
the height of the pancake geometry presented a challenge, as the brightness increas' obi ined by reducing the moderator height is to some extent offset by the reduction in viewable area. A tradt f study was performed between these two effects which is described in more detail in [13], evaluating the 1. nact on the flux at the sample for a full suite of instruments, resulting from the compromise bet een ne acreasing brightness of the source and the decreasing brightness transfer of the neutron optical syste- as the moderator height is reduced. The optimal source height was found to be $3 \mathrm{~cm}$, represes ting the global optimum for the full instrument suite. Once the optimum moderator height was determ - ad lu une pancake moderator, it was straightforward to make the same comparison for the butterfly $m$, de ator , which display the same heightdependent brightness. The viewable width of the cold moderator ' $n$ the butterfly geometry is significantly less than that of the pancake moderator, but this turns ous not to e a problem, since the width of the cold pancake is much greater than can be usefully accepted $b_{y}$ the instruments guide system anyway. The viewable cold surface area of the $3 \mathrm{~cm}$ tall butterfly mode ${ }^{+}$or is approximately $25 \mathrm{~cm}^{2}$, with some variation with beamport angle. This is sufficient for most instr ... th In the case of instruments where a larger area would have been beneficial, the increase in source hrigh ess, compared to the alternative moderators with the larger area, generally more than compensates fo. thr reduced brightness transfer of the system.

The feedback of these phase-space require. ^nıs into the moderator design was that the extraction surfaces for both thermal and cold moderators should be at least $6 \mathrm{~cm}$ wide. As discussed below, the requirement of bispectral extraction and $r$ the $\mathrm{m}$ nber of beam lines, forced the cold and thermal extraction areas to be adjacent.

\subsection{Number of beamlines}

The ESS moderators need tc perı ${ }^{+}+$the extraction of beams for 22 instruments [1]. The instruments are arranged next to each other ${ }_{1} \mathrm{e}$. perimental halls, such that the beamlines need to be arranged in one or more horizontal fans illuminau the space made available in the halls. Existing facilities typically hardwire the number of instrume is i to the design of the monolith, by having exactly the number of beamports built into the target monolı ' $c$,rresponding to the number of instruments to be served by the moderators. Given the typical la eral siz of neutron instruments and their distance from the target, this has generally resulted in approxima. ${ }^{\prime} \sim 2$ beamports being designed into the monolith, separated by about $12^{\circ}$.

ESS has tak n a veı different approach, in designing 42 beamports into the target monolith with an average angular sp. 5 of $6^{\circ}$. This creates a grid of beamports, from which the 22 beams needed for the currently fort. ae scope of the ESS can be extracted, while the remaining 20 beamports will be sealed with temporary dumn. plugs. This has the advantage that the facility layout does not need to be determined for its lifetime by the choice of the initial instrument suite. The monolith design with the 42 beamport inserts 
is shown in Figure 7.

The experimental halls of ESS are divided into 4 sectors, of which two (North and $\mathrm{L}$ st) are similarly sized to existing facilities and will accommodate instruments up to about $60 \mathrm{~m} ; 1$ le. gth. The beamports in these sectors have an angular separation of $6^{\circ}$ between each. Given the amoun $f$ space needed for the instrument components, notably the shielding around the secondary spectromster, - struments of less than this length will typically need to be separated by about $12^{\circ}$, which will be $a^{\prime}$ niev $d b_{v}$ activating, on average, every other beamport.

The two other sectors (South and West) can accommodate instru nents $u$ ) to $170 \mathrm{~m}$ in length. Even instruments with large secondary spectrometers and shielding can e ${ }^{-} \stackrel{-}{-}$ y acuusmodate instruments on neigh-

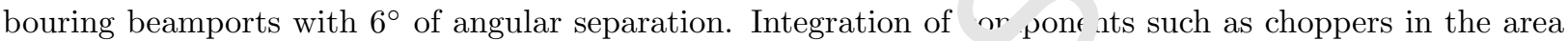
close to the monolith on such closely separated neighbouring beaı. 'ines does, however, present a significant challenge. In order to keep this difficulty at a tractable leve the b' amport separation in the South and West sectors has been staggered to be alternately $5.7^{\circ}$ and 6.0 In this way, each instrument can primarily concentrate on its integration with the neighbouring insı 'ment on the closer beamline.

The ESS design is thus more than sufficient for $t^{\prime}$, n instruments foreseen within the current scope of the ESS, and leaves a lot of room for future possihilities

\subsection{Spectral freedom}

The source frequency is one of the most fundamental parameters around which all instruments at a pulsed source need to be designed. The choice of $14 \mathrm{~Hz}$ is the repetition rate at ESS was made so as to allow each instrument to benefit from a large bandwia ' of ıeutron wavelengths without suffering from frame-overlap, or needing to suppress pulses, as is c $\mathrm{mm}$,nly done on higher rep-rate sources such as SNS or ISIS-TS1. In order to make the most of this av lable " dwidth, most of the thermal instruments at ESS choose to use cold neutrons as well. This is to pican done using a bispectral switch, a device incorporating one or more neutron mirrors at the front $\epsilon . \mathrm{d}$, $f$ the instrument's neutron optical system, inclined so as to reflect in cold neutrons from a source to the - de of the guide axis. The device acts as a switch because the reflectivity of neutron mirrors is int insi ally wavelength-dependent, so that short wavelengths are transmitted while longer wavelengths are retı +er'. Without needing to move, it thus switches as a function of wavelength from allowing short waveles th neutrons to be transmitted until the reflectivity cut-off is reached, above which longer-waveleng. $n$ atrons are reflected into the guide from the side. Together with the choice of the rather low : spetitio rate of the ESS, a decision was made early on in the project that all beamports should be able to n . it from the resultant large bandwidth by using bispectral switches. This has farreaching imp 'a' ans on the design of the facility. Because every beamport can now be populated with a cold, bispectra. or purely thermal instrument, every beamport becomes essentially equivalent, and an instruments choice of beamport is no longer based on its spectral needs, as is done at existing facilities 
but on other factors, such as the size of the experimental hall behind that beamport, he space allowed by components such as choppers on the neighbouring instruments, or proximity to la? s anc ther important infrastructure.

Currently existing pulsed spallation sources hardwire the spectral characteristı of each beamport into the monolith design by orienting the beamport axis to point at one moderator or at ther. Adding this new requirement of spectral freedom to all beamports requires a very differen apr oav... Each beamport now needs to be able to view both a cold and a thermal neutron source, which rhous he placed side by side with the beamport axis oriented at their junction. In this way, the instrum nt can ' hoose to shift and/or tilt its beam axis with respect to the beamport axis within the envelope $r$ en $\mathrm{vy}$ une beamport insert, so that it points at the cold or thermal moderator. The design of the bean m $*$ ins rts shown in Figure 7, typically allows the beam to be tilted by about $1^{\circ}$ to one side or the other, rith respect to the beamport axis.

For the TDR moderator, this works fine. All beamports 1 . nne 60 opening should be oriented to point at the junction between the cold and thermal moderators, an ' the instruments can choose cold, bispectral or thermal beams by tilting one way or the other.

For the pancake moderator, there are two option : n shieving this spectral freedom. Each beamport can either be oriented so as to point at the cold-thermal, nction nearest to its entrance window or the coldthermal junction on the other side of the cold modt au - These are known as near-corner and far-corner extraction, respectively and are illustrated in $\mathrm{H}_{\mathrm{c}}$ '

Near-corner extraction works well for beamlines near the perpendicular to the proton beam, but results in very low thermal brightness at angles close t the edges of the fan, as the beamport angle approaches the parallel to the thermal-moderator face af Section 5.1). This is consistent with the known angular dependence of neutron emission fror the mal moderators [26]. Far-corner extraction works acceptably well for all beamports, but generally $\mathrm{r}$ sults in : wer cold brightness, as the viewing angle of the cold moderator for most beamports results in a redu $d$ depth of parahydrogen being seen. The other problem with the far-corner extraction is that; res ilts in fewer beamports overall, as the beamports near the perpendicular to the proton beam would othe. rise clash [13].

The butterfly geomet $\mathrm{y} \mathrm{sr}$,ves this problem by orienting all beamports towards the nearest cold-thermal junction. For each instrumt. ${ }^{2}$,ector (North, West, South, East), this junction acts as the focal point for the beamports, as ir licated y the red spots in Figure 25. All beamports can view either the adjacent cold lobe or the therm ${ }^{-1}$ mou u uror on the other side of the focal point, as indicated by the arrows in Figure 26. The butterfly-1 xeomet , has an advantage over the butterfly-2 geometry for thermal beam extraction, as the central, most inuuse part of the thermal moderator near the inside of the $V$-shape is closer to the focal point, making 't more readily viewable from most beamports. 


\subsection{Upgradeability}

All three of the studied low-dimensional moderator concepts incorporate a high degree c." 'pgradeability, as two upgradeability aspects were included as design criteria from the outset: $\mathrm{f}$ stly the beam extraction geometry has been designed to allow the extraction of many more beamports than $\mathrm{h}_{\text {- }}$-red for the 22 instruments foreseen in the day-one configuration of the ESS. The primary upgr ' palı of the ESS is to add more instruments to the initial target station, rather than needing to bu $\mathrm{i}$ a secoud target station, when the 22 instrument slots have been filled, as is the strategy at existing ised sp llation sources. This is a far more cost-effective upgrade path, capitalizing on the high perforn ænce of ine existing accelerator and target station, without the large added expense of a second target sation and the dilution in proton beam power associated with the need to supply beam to two target stai ${ }^{\circ} \mathrm{s} \mathrm{wi}^{+} \mathrm{a}$ the same accelerator. In order to facilitate such an upgrade, the angular separation of the beam ${ }_{r}$ rts at ESS has been set to about half that of existing pulsed spallation sources, resulting in up to $4\llcorner$ `ossib e beamports, see Figure 7.

Secondly, the design requirement that all beamports can s- oly choose between viewing the cold and thermal moderators provides a significant additional freea ${ }^{n}$ of choice when placing future instruments. In addition, contrary to current pulsed spallation source $w_{n} \cdot$ ' ise a combination of coupled, decoupled and poisoned moderators to provide the range of pul `wid ' characteristics needed by the instrument suite, the ESS as a long-pulse source, provides a much gre 'er $\_$nge of pulse widths by tailoring the source pulse on an instrument-by-instrument basis using puls -hapıng choppers on all the beamlines which need them. This range is available to all instruments, bnth for day-one instruments, and for instruments which are part of a future upgrade. Finally, by allowing all the istruments to be served by the same moderator assembly above the target, the space for moders ors be. the target is kept free to be used for future developments. Some ideas are briefly discussed in $t$. a ncly ,ion section, as well as in Ref. [13].

One aspect of the target stat: in desig ${ }_{\Lambda_{1}}$ which does impose limitations on future moderator designs is the location of the four focal points. I I focal points are intended to be fixed for the lifetime of the facility, as the beamport inserts are; 1 teg ated into the whole target-monolith system. The height of the beamport inserts is set so as to allor a nt ' ${ }^{2}$ on beam to be extracted from either the top or the bottom moderator, or even both. The horiz nta' coo' linates of the focal points thus apply equally to both the top and bottom moderators and are fi $\ldots$ i for $i$. a lifetime of the facility.

\subsection{Iterative procedum ${ }^{f \sim m}$ moderator optimization}

The conside ations c atlined above for optimal beam extraction need to be incorporated into the design of the moderators. \& ESS, this was approached in an iterative fashion. The requirement on the viewed surface at the $\eta$,derator, for the ESS beam extraction configuration, was that a width of at least $6 \mathrm{~cm}$ was needed, for both hermal and cold extraction surfaces [13]. Such a width should be seen from each of the beamports, and was therefore treated as a requirement for the moderator design. 
Figure 12. Calculated brightness $B_{0-20}$ map on the emission surface of cylindrical moder tors - different heights: $1.5 \mathrm{~cm}$ (top), $3 \mathrm{~cm}$ (center), and $10 \mathrm{~cm}$ (bottom).
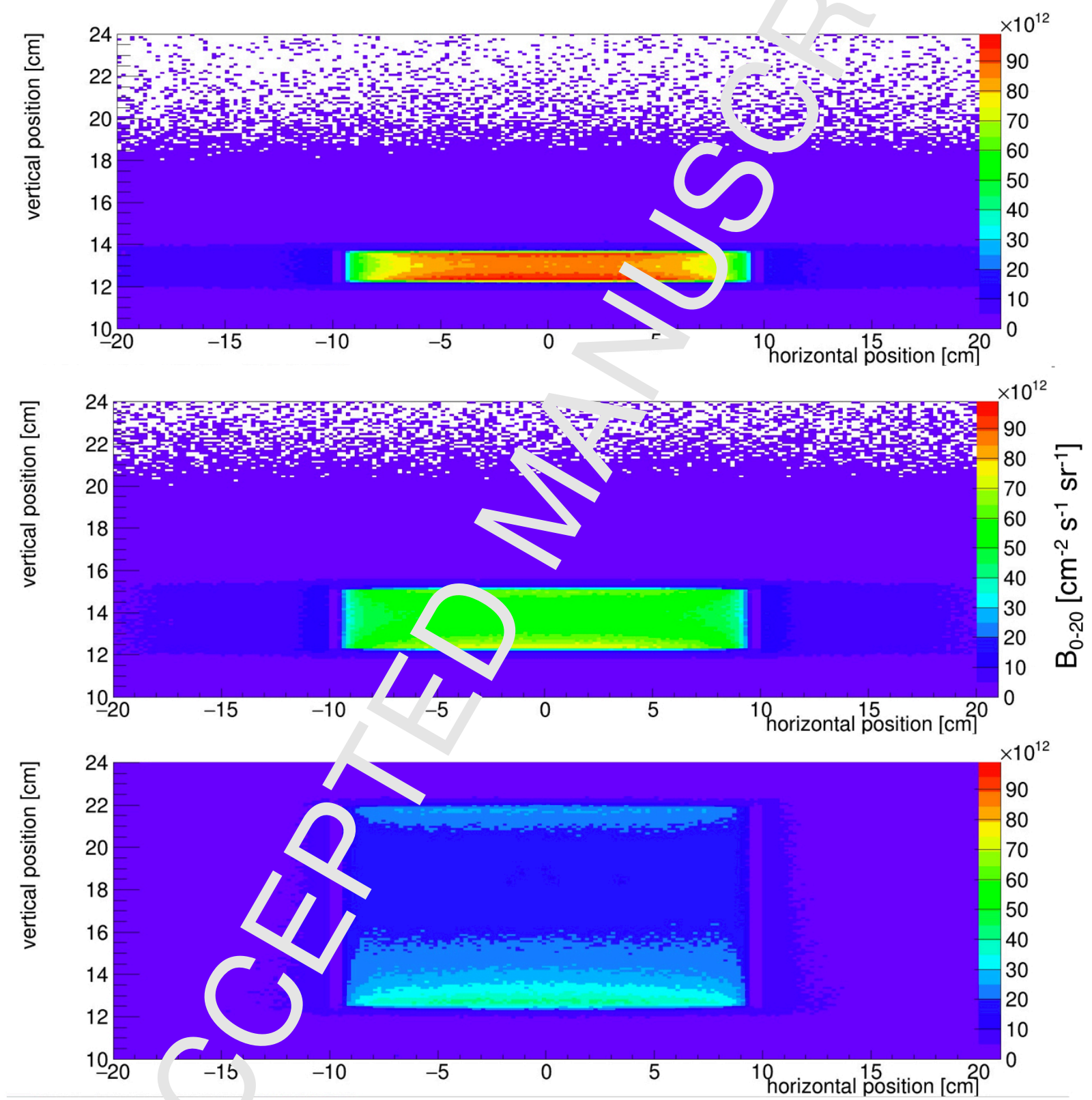
Figure 13. Same as Figure 11 for $B_{0-20}$.

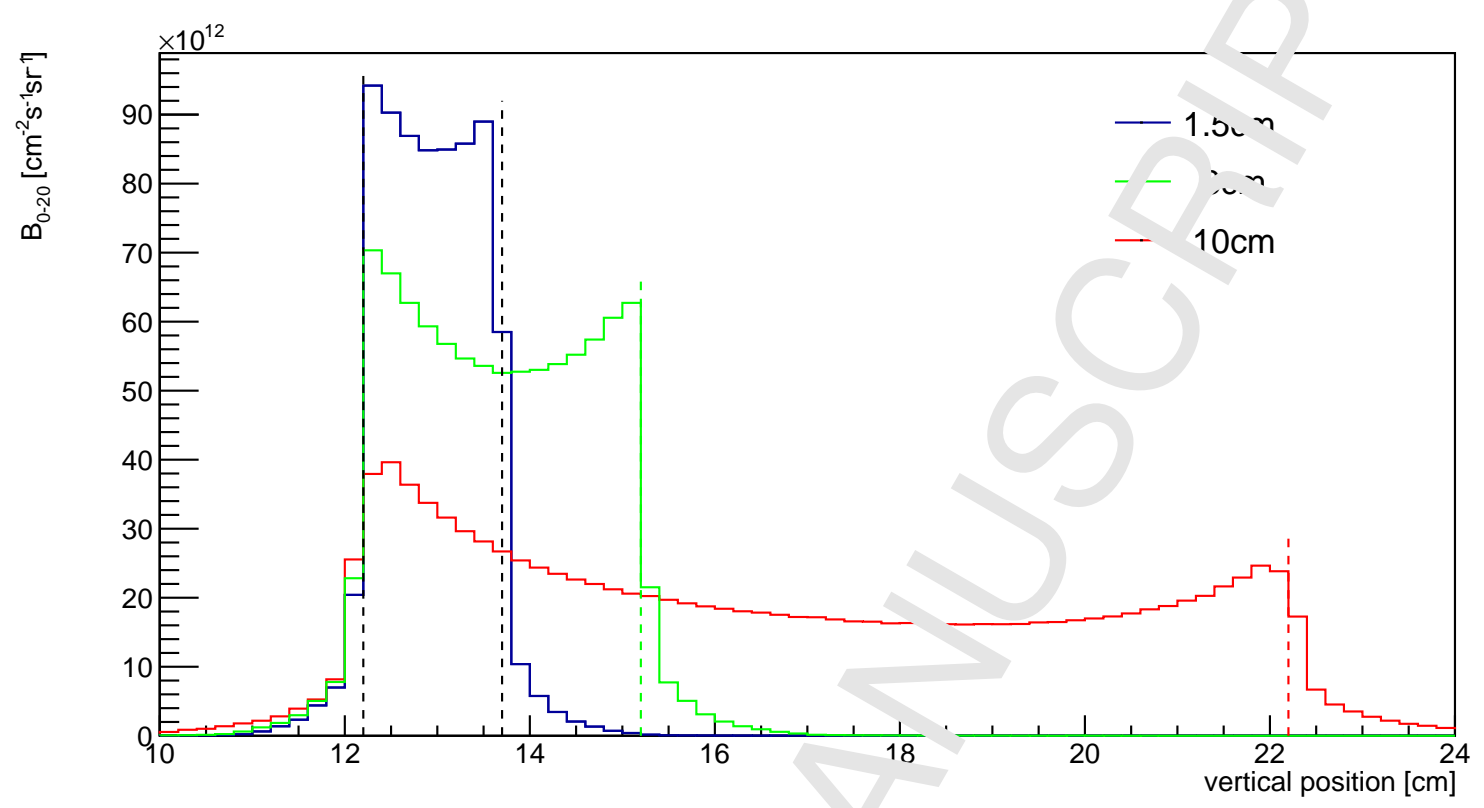

Figure -t. ' 'me as Figure 11 for $B_{0-5}$.

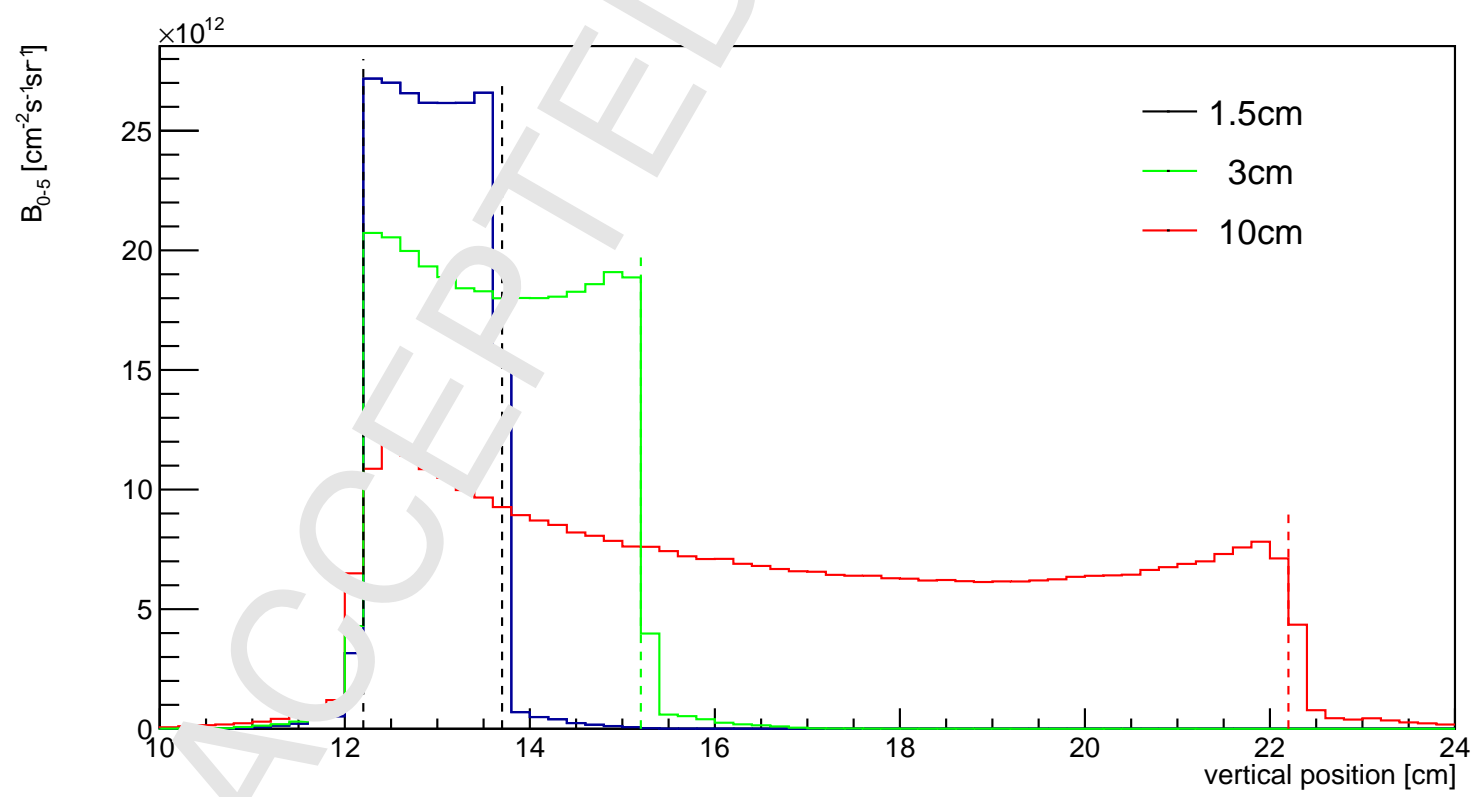


Each moderator design as it was proposed to the instruments was evaluated for pe formance impact on each instrument, and the result was fed back to the moderator design team. At each terav $\backsim$, the strengths and weaknesses of each particular moderator design for instrument performance ere highlighted, allowing an informed adaptation of the design to the instrument needs. This allowed the $\omega_{r}$ mal moderator height to be calculated, so as to maximize the global performance of the instrument sulv. The issues related to beam extraction were also evaluated at each iteration, culminating in $t\} \_$bv, tes $-y$ moderator geometry which takes all the relevant performance parameters into account.

According to these criteria, the chosen moderator height was $3 \mathrm{~cm} \mathrm{f} \mathrm{r}$ both 1 lermal and cold moderators.

\section{Moderator Design Choice}

\subsection{Relative performance of the different concepts}

In this section we compare the performance of the three `ncepw considered for low-dimensional moderators. The brightness was calculated from a viewed surfa $\ldots \ldots$ unoderator $6 \mathrm{~cm}$ wide, $3 \mathrm{~cm}$ high. This was done for both thermal and cold moderators and the requiremı nt in the calculations were that each viewed surface would be at a distance of at most $1 \mathrm{~cm}$ from tı, $f$, scal point.

For the pancake, the dimensions of the cold $h_{\nu}{ }^{\prime} \omega_{e n}$ disk that gave the highest cold brightness was chosen, for both near and far corner extractior

For the butterfly- 1 and butterfly-2 moderators, he total length of the moderator system (along the proton beam axis), after optimization, is $\mathrm{st} \mathrm{Z}^{\circ} \mathrm{m}$ and $28 \mathrm{~cm}$, respectively. For the pancake, the optimal diameter of the cold moderator disk is $20_{\mathrm{c}}$ n. for ooth near- and far-corner extraction. The overall pancake moderator is, however, wider than $\mathrm{k}$,th ' utterfly-1 and butterfly-2, as the $6 \mathrm{~cm}$ wide thermal extraction areas must be considered, on both side of + 1 e cold moderator, making the full pancake moderator system about $32 \mathrm{~cm}$ wide.

The Monte Carlo models $r$.u ot have full engineering details, as they were not available at the time of performing the optimization $+\cdots, y$. However, in an attempt to estimate absolute values of the brightness expected, several details are ncluced such as pipes, correct material thicknesses, and gaps, while some materials are mixtures in - der o take into account water loops for cooling, or flow guides in hydrogen or water moderators.

The three low-dir nnsir al designs (one pancake and two butterflies) had exactly the same level of engineering det: $1 \mathrm{ls}$, allc ing a proper comparison of the different concepts. The target, reflector, and all the surrounding of the mn erators were identical, and the only difference between the models were the thermal and cold mo 'ere us inserted. The flow channels inside the moderators were not modelled, instead the material inside $\mathrm{v}$ s a mixture of parahydrogen with $5 \%$ of aluminum to take into account the reduction in performance due to the presence of alumimum flow guides inside the moderator vessel. 
In the case of the TDR volume moderator, in order to perform as fair a comparis in as possible to the other models, we took the model used for the TDR work, and added some enginec ing it ails (e.g. same structure and amount of cooling water in the reflector) to make it similar, from the engineering point of view, to the latest low-dimensional models. We also considered the same type ' ' beam extraction, i.e., within $7 \mathrm{~cm}$ from the focal points, placed at the intersection between thermal anu nld sources, as for the latest models.

Despite the addition of engineering details, this updated TDR model is nroba ${ }^{1} \mathrm{v}$ still favored with respect to the other concepts. Since the publication of the TDR, the spallatior target 1 as been redesigned in favour increasing the volume for the flow of the helium coolant, and the mr sratus nave been moved further away from the target to increase the safety margins. These changes $r^{1} 1^{1}$ in : reduced neutronic performance close to $10 \%$. They have not been incorporated in the updated 1 . $\mathrm{R}$ model presented here.

Figure 27 shows the resulting brightness angular distribuı ns witl $n$ one $120^{\circ}$ sector of ESS for the lowdimensional moderators considered. Due to symmetry, the res. ${ }^{1+}$ s are identical in the other sector. Integral thermal $B_{20-100}$ and cold $B_{0-20}$ brightnesses are showı 'or the pancake geometry (with near-corner and far-corner extraction) and for the two butterfly confic ... Linns.

Table 2 lists the average integrated thermal and cold x ightness for the designs considered. From Table 2, it is apparent that all the low-dimensional models ha. a much higher brightness than the TDR, thus justifying the transition from TDR to low-dimes. 'ollai noderators.

Of the two beam extraction concepts for the pancake geometry, the far-corner option is preferable, as it offers a larger gain in thermal brightness ihough it results in the loss of a number of beamports. Reduction of each pair of beamports can be seen is a $14 \mathrm{~s}$ i available neutrons to the facility, considered as whole, of about $5 \%$. The near-corner extractic of 10 \% or the pancake provides a higher cold brightness and does not

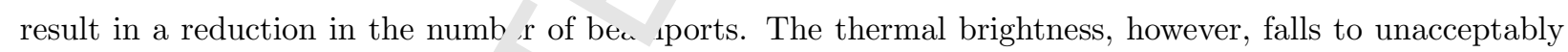
low levels for the edge beampor.s.

The two butterfly concept, we a designed with the goal of increasing the thermal brightness, maintaining (or increasing) the high crld $\mathrm{v}_{\text {- }}$ rhtness, and easing the beam extraction. Of the two concepts, the most successful is the butterf 1 , Jeca`ise; $i$ ) it has the highest cold brightness of all concepts. More than two thirds of the instruments of ${ }_{2} \mathrm{Cr}$ are cold or bispectral, therefore in the overall figure of merit to choose the best concept, cold $\mathrm{b}$. ghtness must have the priority. $i$ i) It has the second best thermal brightness, after the butterfly 2, which ' Jwe ... nas a significantly lower cold brightness.

Compared $t$ the pa cake with far-corner extraction, the butterfly 1 gives both higher thermal and cold brightness (factor $1.1 v$ and 1.12, respectively), without the problem of the loss of beamports. The real gain is however his ${ }^{\prime} r i$, considering the higher number of beamports available for the butterfly 1 concept, as discussed. Compcred to the pancake with near-corner extraction, it gives a slightly higher cold brighness, and an average thermal brightness higher by a factor of 1.47 . 
Figure 15. $B_{20-100}$ distribution across the moderator height, for thermal neutrons. Neutrons are extr - ted from the water-filled sides of three moderators of cylindrical geometry, with $1.5 \mathrm{~cm}, 3 \mathrm{~cm}$ and $10 \mathrm{~cm}$ height.

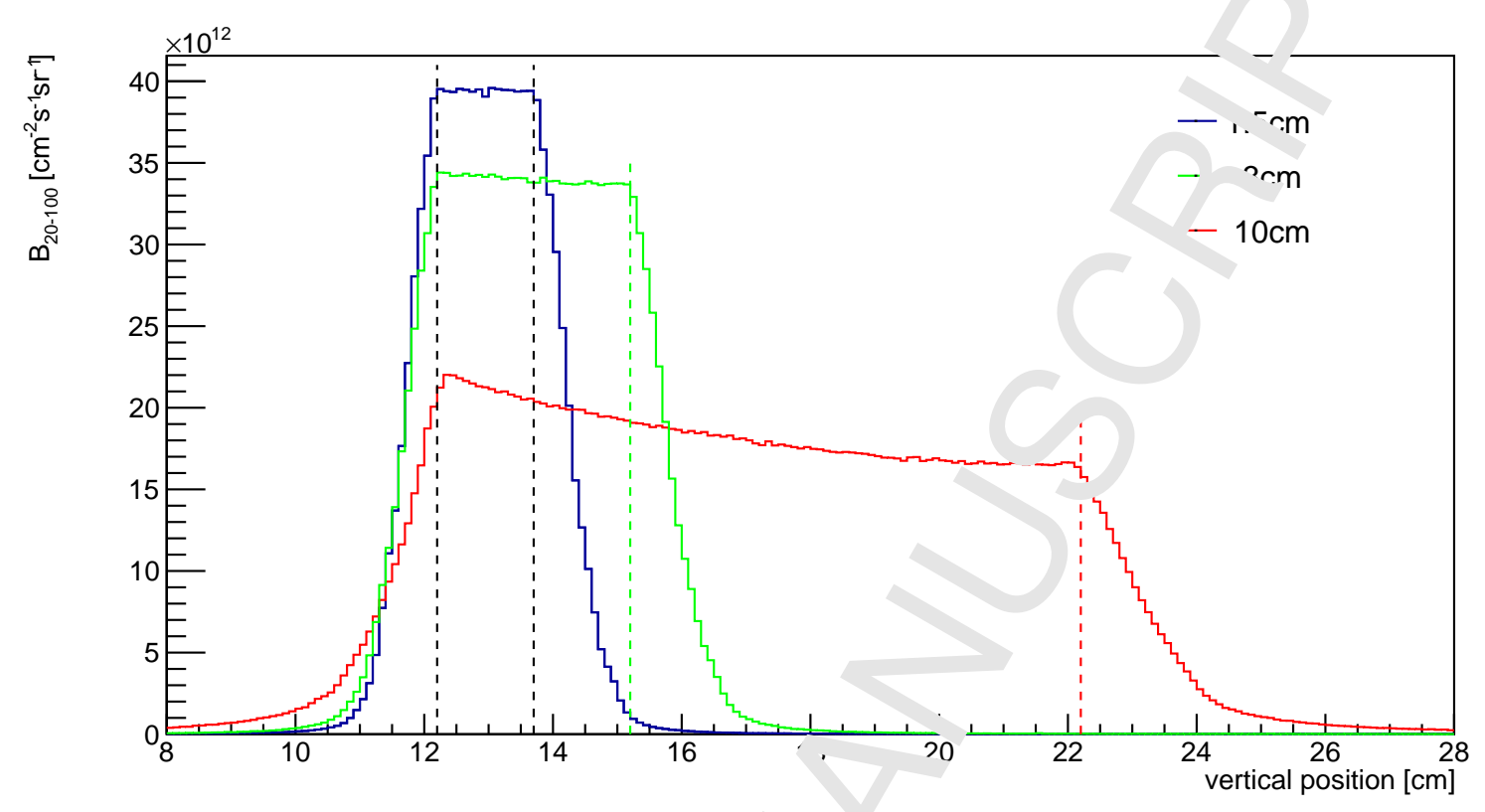

\section{Description and performance of the butte. ${ }^{\top}$ y 1 moderator}

The first moderator that will be instal' $\mathrm{d}$ at $\mathrm{E} i \mathrm{~S}$ at the start of the facility will be a butterfly 2 moderator, which was initially chosen mainly beca se of $\iota_{\text {. }}$ igher thermal brightness. At the time of writing, a detailed engineering design has been finished $n$ nd the toderator is in fabrication for installation in 2021. For a final configuration of the top moderat ir we de ded to adopt a butterfly 1 design, for the reasons outlined in Section 5. The instruments and the butterfly 1 system will be $\mathrm{i}$ stal $\mathrm{d}$ in the early years of ESS during the power ramp-up. That is why detailed engineering mode' 3 are - ot yet available for the butterfly 1 system.

In this section we gi e a all $r$ escription of the butterfly-1 moderator chosen for ESS. The geometry of the butterfly moderat $\quad$ is sh $\quad 1$ in Figure 25. Engineering details have been extrapolated from the existing engineering model o the bui erfly 2 [27].

The MCNPX moder ur the structures surrounding the moderator is shown in Figures 28 to 30. Some important detai ; of the model are the following:

- The tu^ rst $\mathrm{a}$ uarget was modelled according to the engineering drawings. The density of the tungsten used in the nodel is of $15.1 \mathrm{~g} / \mathrm{cm}^{3}$, corresponding to the effective density deduced from the engineering design, which consists of tungsten bricks with cooling gaps, for a filling factor of tungsten of $78 \%$. The 
Figure 16. Angular distribution of propagation direction with respect to the horize tal plar 2 of cold neutrons $(E<20 \mathrm{meV})$ emitted from the surface of the parahydrogen moderator.

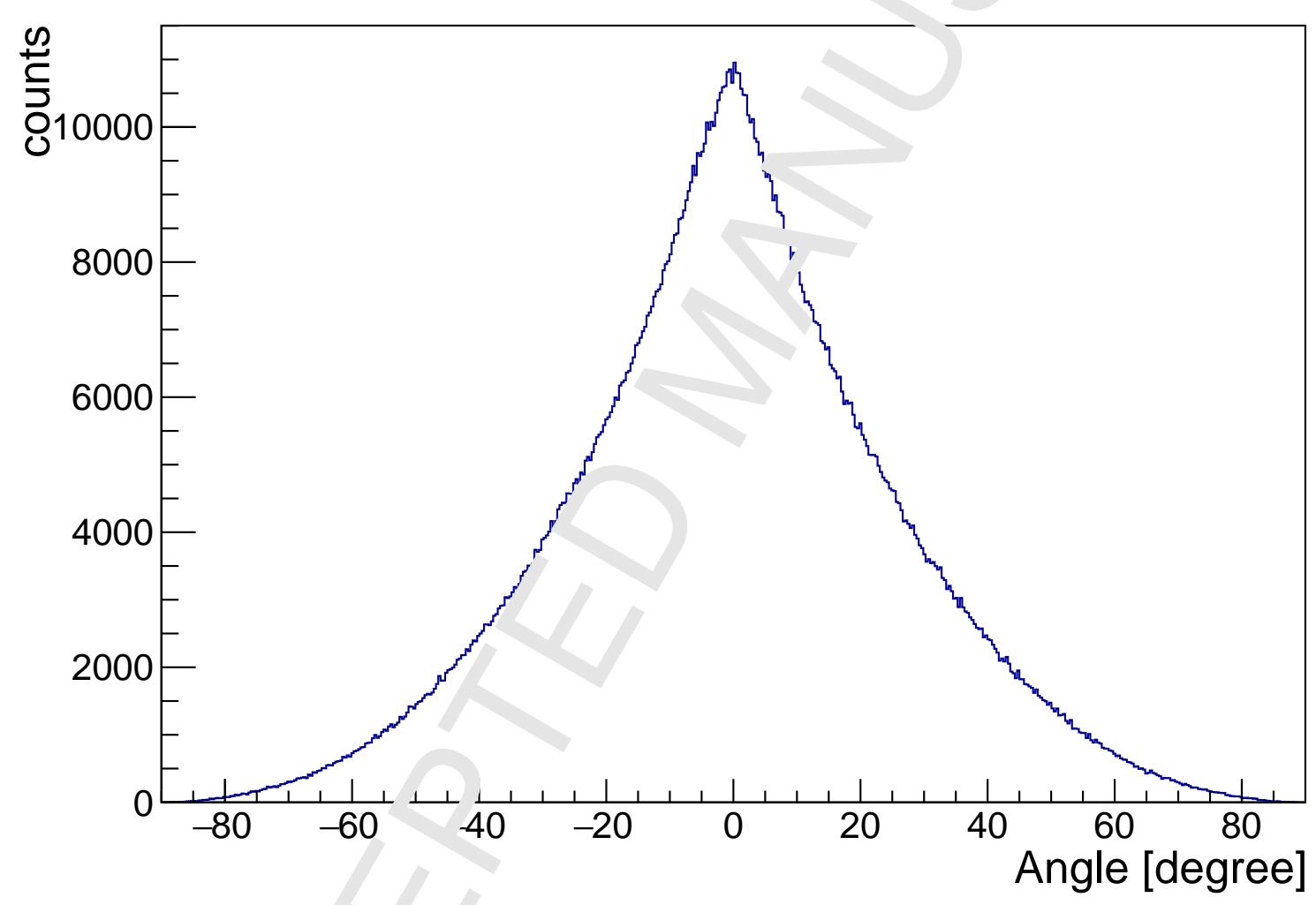


Figure 17. Angular distribution of the propagation direction with respect to the ho rontal $\mathrm{f}$ ane of thermal neutrons $(20 \mathrm{meV}$ $<E<100 \mathrm{meV}$ ) emitted from the surface of the water moderator.

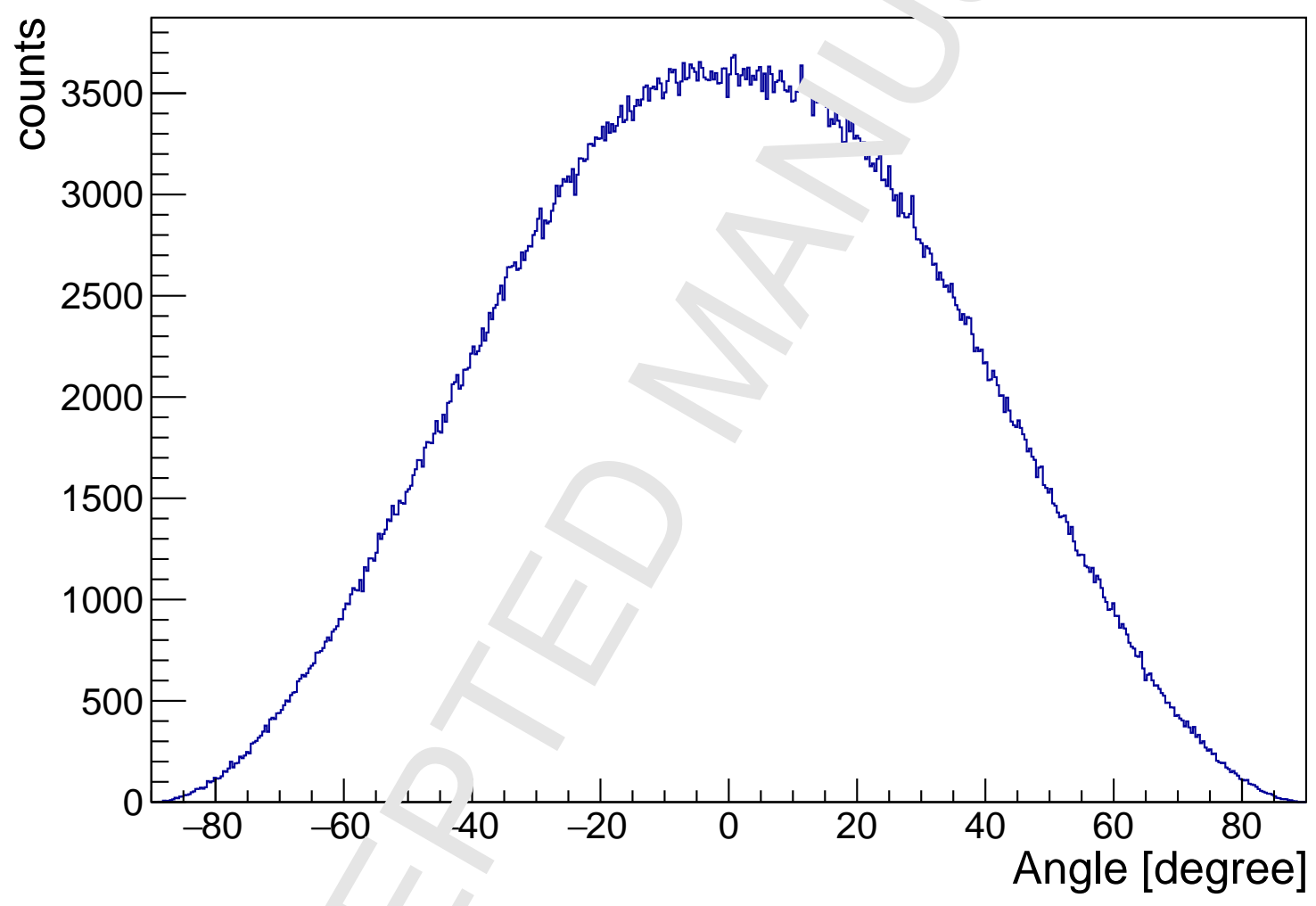




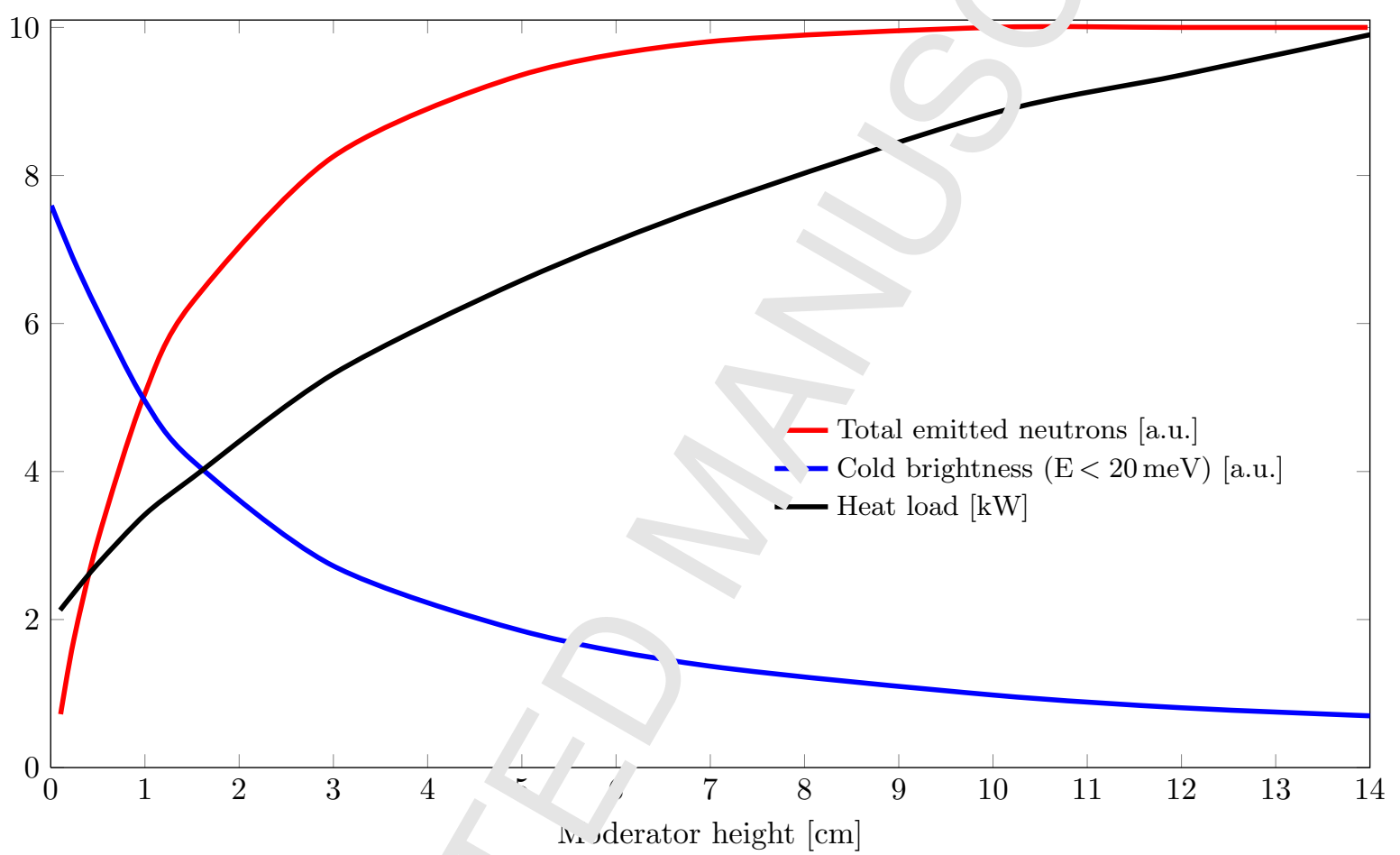

Figure 18. The integral cold bright ess $30-20$ increases with decreasing height of the moderator. On the other hand, the total number of emitted cold neutrons, $?_{n}, 0$ multiplied by the viewed area of the emitting surface) and the heat load both increase with the moderator height. T he heat. $\mathrm{d}$ is calculated on the cylindrical aluminum vessel and on the pure parahydrogen inside, without piping or ot $\mathrm{r}$ er, inee ng details. 
Figure 19. Relative change in thermal and cold brightness as a function of the pre loderatoi radius, for two configurations, in which the ring outside the premoderator (with inner radius equal to the premodera. $\cdots_{m} . .4$, and outer radius of $60 \mathrm{~cm}$ ) is void, or beryllium with 30 vol\% water.

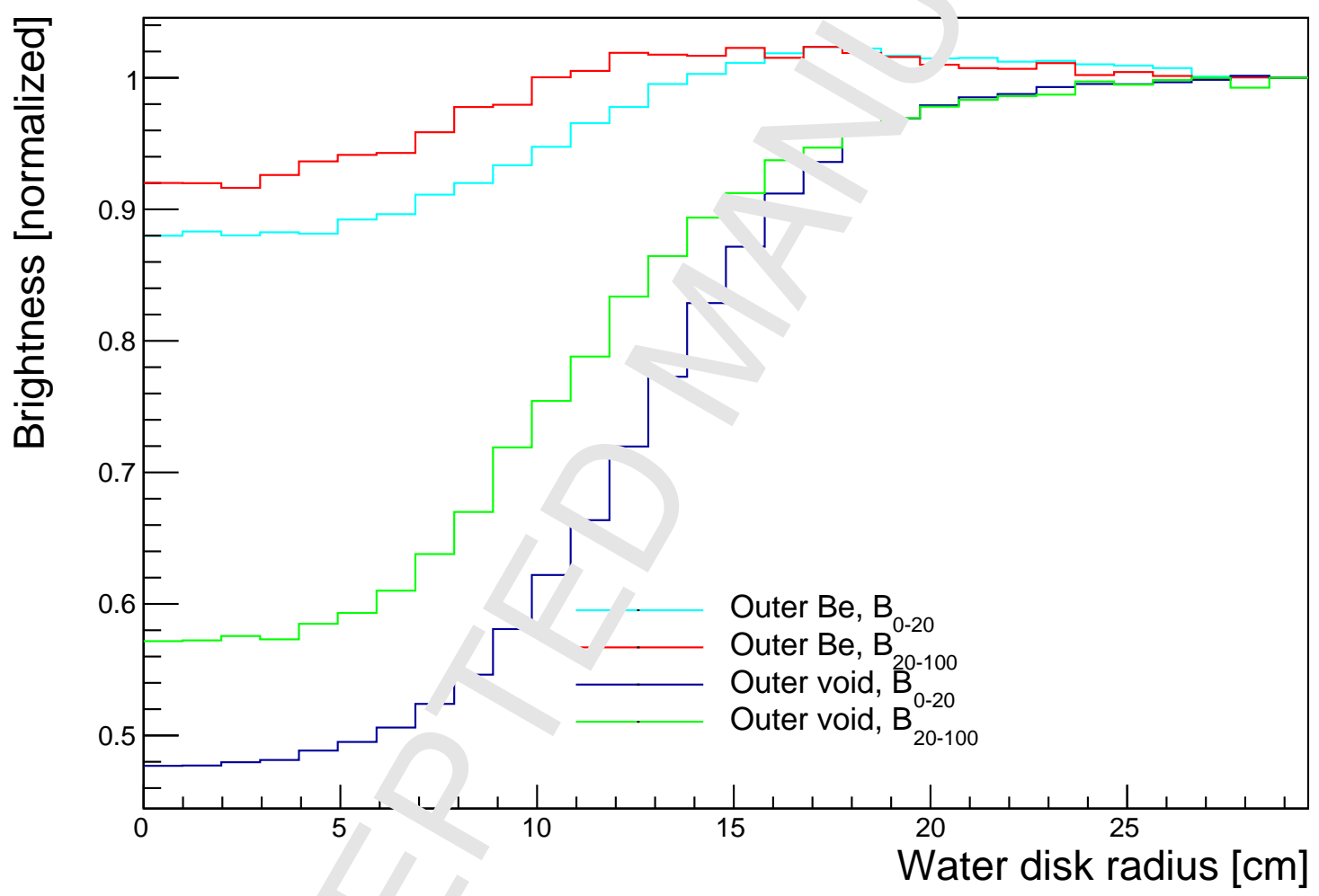




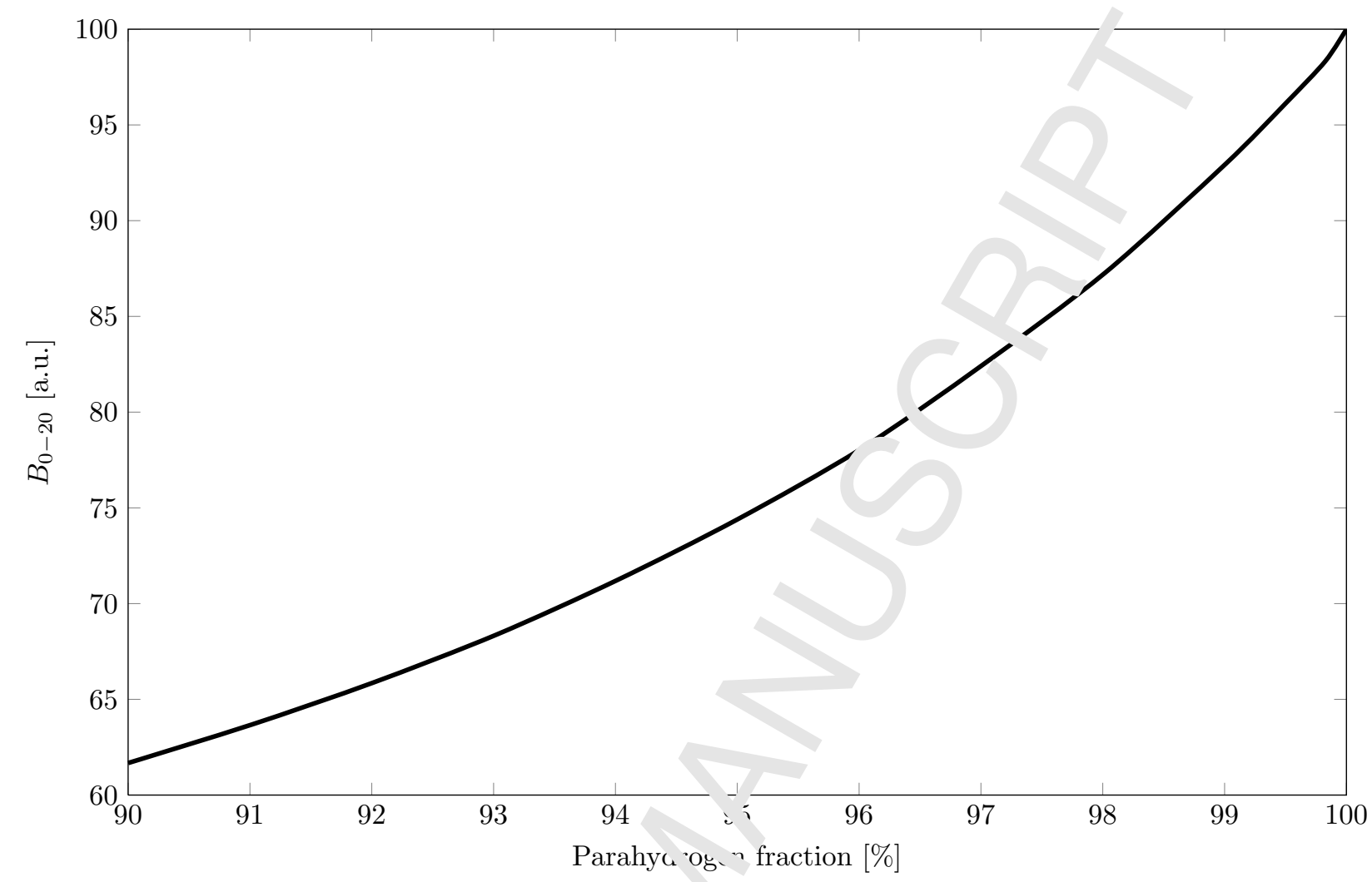

Figure 20. Brightness $B_{0-20}$ relative variation, for the $3-\mathrm{cm}$. ; cold moderator, as a function of the parahydrogen fraction, calculated using the ENDF-B/VII cross section $\mathrm{a}^{+}+\mathrm{w}^{-}$

Figure 21. Map of high-energy $\left(E>0.1 \mathrm{Me}^{\top}\right)$ neu $\neg n s$ crossing a horizontal plane located between the tungsten target and the premoderator, at $Z=8 \mathrm{~cm}$. The shape of + 1 e butterfly (left) and pancake (right) moderators are superimposed. (The proton beam direction in the figure is up varu.
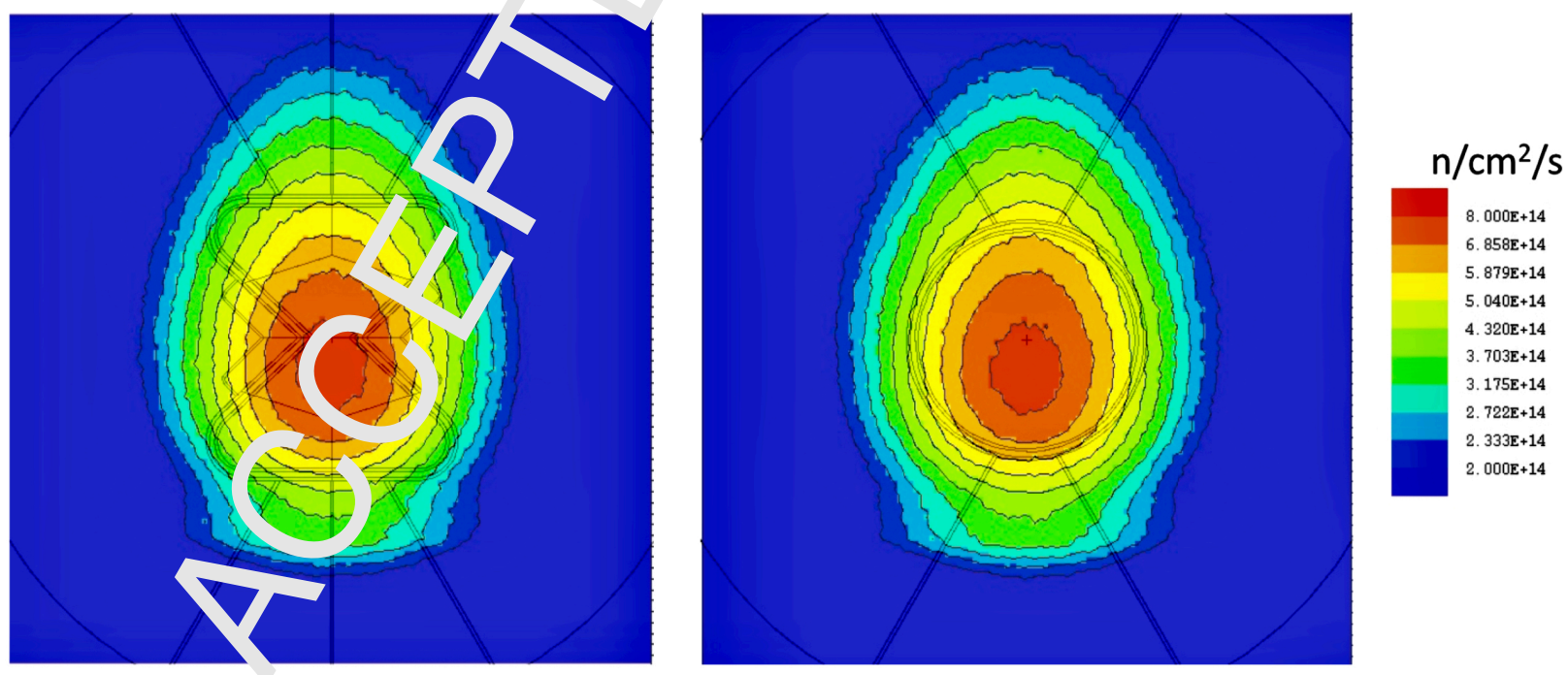
Figure 22. The fast neutron emission distribution for the $1.5,3$ and $10 \mathrm{~cm}$ tall ancake 'oderators The results have been obtained by a point detector tally (f5) with the emission position (horizontal axis, $0=\mathrm{c}$ nter of arget) calculated by extrapolating the trajectories back to the center of the moderator. Neutrons shown as starti $s$ outcide the window defined by the moderator edges (dashed vertical lines) traverse through the structure around the mode. $t$. that is aligned to the physical edges of the moderator volume.

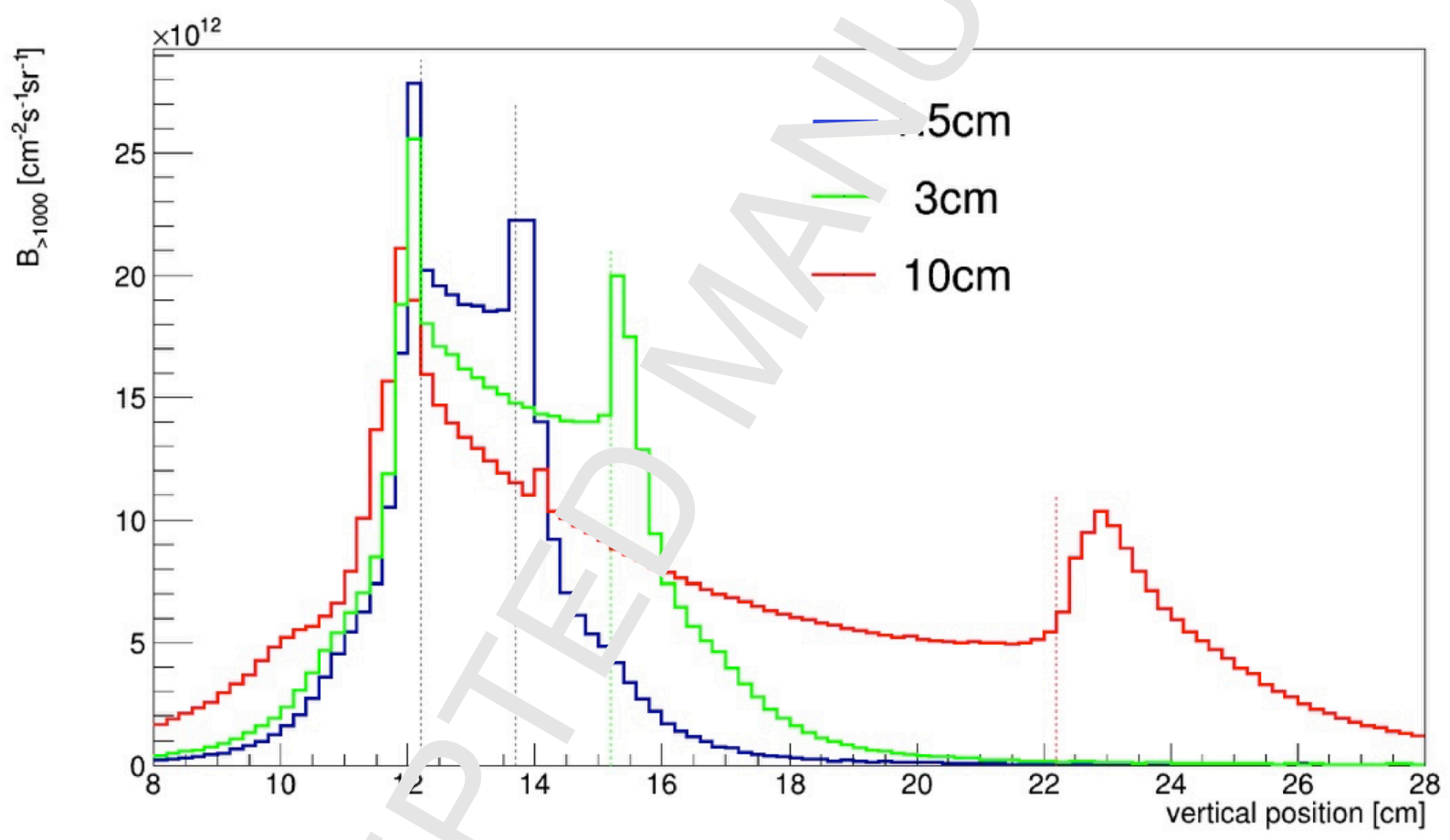


Figure 23. Evolution of the relative signal to fast neutron background ratio $\left(S / I_{F}\right)$ and he data collection figure of merit $(\mathrm{FOM})$ as a function of moderator height. For definitions see the text in Section 2.

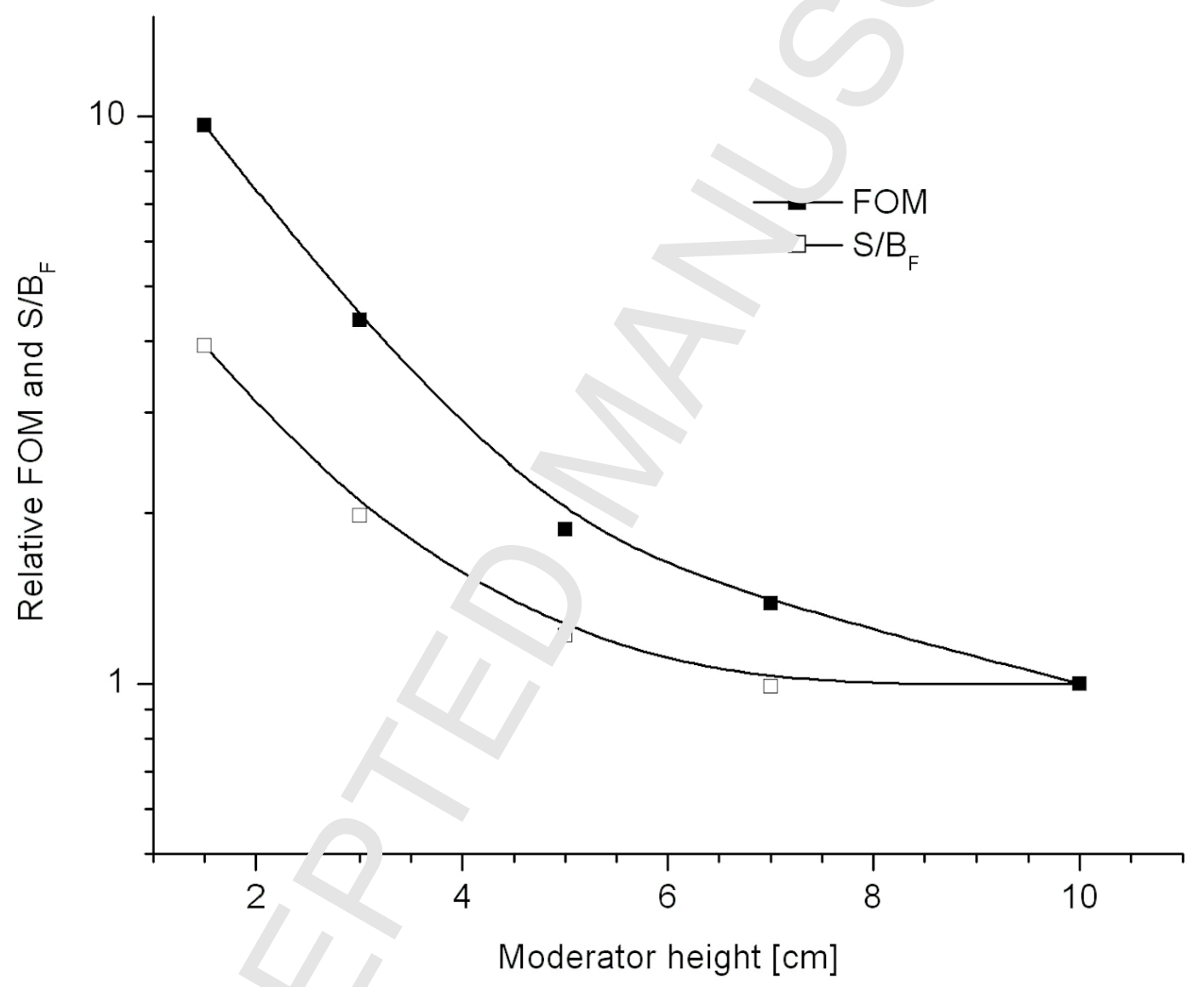


final brightness was calculated using a model with the individual bricks, and $\mathrm{i}^{\prime}$ was verified that it gives the same brightness as the homogenized model. The SS316L container of ne $b_{1}$. $k^{k}$ s was modelled in detail according to the engineering drawings.

- The water premoderator between target and moderator consists of an ain ninuı vessel, containing a mixture of water and aluminum, with a volume fraction of $\mathrm{Al}$ of 8 ol; to account for water flow channels, deduced from the engineering drawings of the water premou ${ }^{-9}$, or currently in construction.

- Similarly, parahydrogen in the cold moderator contains 5 vol\% o alumin $\mathrm{m}$, corresponding to the expected volume fraction occupied by the flow channels. The fin ${ }^{1}$ mo ${ }^{\prime}$ ' uwever contains a preliminary design of the flow channels [28]. The shape and thickness of 1 1 - um tum vessel is modelled according to the engineering drawings.

- Thicknesses of aluminum walls of the cold and thermal mo Jera ors, and of the beryllium reflector are in agreement with the engineering drawings. Similarly, thr heryllium reflector is modelled according to the present status of the engineering design, with i. a expected amount of light water cooling.

- The outer reflector is made of SS316L with 10 vo.7\% of water cooling.

- Two hydrogen pipes, inlet and outlet, are assun ed or the cold moderator, see Figures 2528 and 30

The results provided are with a fast neutron refle tor placed at the bottom of the target. For the first moderator-reflector plug, with a butterfly 2 mu 'orator at the top, a steel plug is designed for the bottom, which gives a $4 \%$ brightness increase wlı. resr ect to having a two-moderator configuration. The result presented here are for a reflector prc jidir $\mathrm{g}$ a $9 \%$ brightness increase which could be achieved by a watercooled beryllium or copper reflectc, whı a setter performance could be obtained using other options, such as helium cooled tungsten (cf. T „ $\mathrm{blt}^{1}{ }^{1}$ ).

\subsection{Focal points}

The focal points are d' ferf at for the four instrument sectors. The focal points are (for the North sector) $X=5.4 \mathrm{~cm}, Y=8.9 \mathrm{~cm}, \mathrm{Z}-3.7 \mathrm{~m}$. For the other sectors, the absolute values of the $X$ and $Y$ coordinates are unchanged, but ${ }^{\dagger}$ ne sigr change according to the sector (see Figure 25).

\subsection{Horizontal ıewed width}

Figure $31 \mathrm{sh}$ 'vs thr projected widths at the moderator surface, for thermal and cold moderators, for the differen ' nean $\cdots$ ts. In general there is a good balance between maximum widths of thermal and cold moderators, as . 'quired for effective beam extraction. 


\subsection{Horizontal brightness distributions at moderator}

In Section 2 the brightness distribution along the height of the 3-cm pancake muderat was shown for several integrated brightnesses. The shapes of these distributions depend mainl on he moderator height and therefore the vertical distributions for the butterfly moderators are simil $r\left(w_{\perp}{ }^{10}\right.$ the absolute values depend on the performance of the moderator).

Horizontal distributions of the brightness at the moderator surface ere also calculated. This work was part of a detailed characterization of the moderator brightness, ' ing us 1 in the ray-tracing code McStas [29], for guide and instrument design.

The geometry and brightness data presented here have been " «plemented in the most recent version of the McStas ESS source component, released as an updated apon at library for McStas 2.3. The implementation is based on functional fits to the underlying $\mathrm{MCN}^{2}$ - data. This approach ensures that the simulations run efficiently, but comes with some simplificatı \& and assumptions, which means that the results are only valid to within about $10 \%$. Users which are $w_{\iota_{2}}{ }^{\prime}$ ng to accept a CPU performance penalty, can instead make use of the MCPL implementation [30], w. ' McStas with no loss of accuracy in the transition bei tetre das.

Horizontal distributions were calculated with $\mathrm{N}$ NPP , using collimators placed at $3 \mathrm{~m}$ distance from the moderator, looking at a surface $2 \mathrm{~mm}$ wide, $30 \mathrm{~mm}$ his., moving the collimator to obtain a distribution across the thermal and cold moderators. Results re snown in Figures 32 and 33.

The brightness distribution across the viewed surface shows variations for different beamports. For example, for the W1/E1 beamports, the old bric nness is low for about two $\mathrm{cm}$ from the focal point, then it reaches a plateau about $5 \mathrm{~cm}$ wide. $T$ is is $\omega_{-}-$to the lower amount of hydrogen viewed at this particular angle, for that 2-cm window (cfr. F. "rr 32 ) Figure 33 corresponds to a beamport at $42^{\circ}$ with respect to the proton beam axis, which is $\mathrm{v}$ ry close $\mathrm{o}$ the $45^{\circ}$ angle of of the cold moderator vessel walls adjacent to the thermal moderator. Therefore $i$ is beamport sees for a few $\mathrm{cm}$ of the extraction window the cold vessel in its entire length acr ss th it diagonal, resulting, for about half of the viewed surface, in a brightness about $25 \%$ higher than fr: the 'ther half; if neutrons are extracted from that $3 \mathrm{~cm}$ window only, the cold brightness would be abr it 1\% \% ' igher than from a $6 \mathrm{~cm}$ wide window.

Thus, for the cold ...odera. r, a significant gain of 10-15\% is observed for 16 out of the 42 beamports. Such effects are not, ossible or the pancake, nor for the butterfly-2, and if exploited by careful guide optics design it can yie' , an aautional cold brightness increase for several beamports.

\subsection{Brightness re.}

In Figure " ne distribution of $B_{0-20}$ and $B_{20-100}$ for the 42 ESS beamports is shown. For 16 beamports we observe a significant increase of the cold brightness. These are the beamports where the quasi-tube 
Figure 24. The TDR configuration. Left: Vertical cut showing top and bottor mod - tors. Right: Horizontal cut showing top cold moderator and water extensions for bispectral neutron beam extraction.
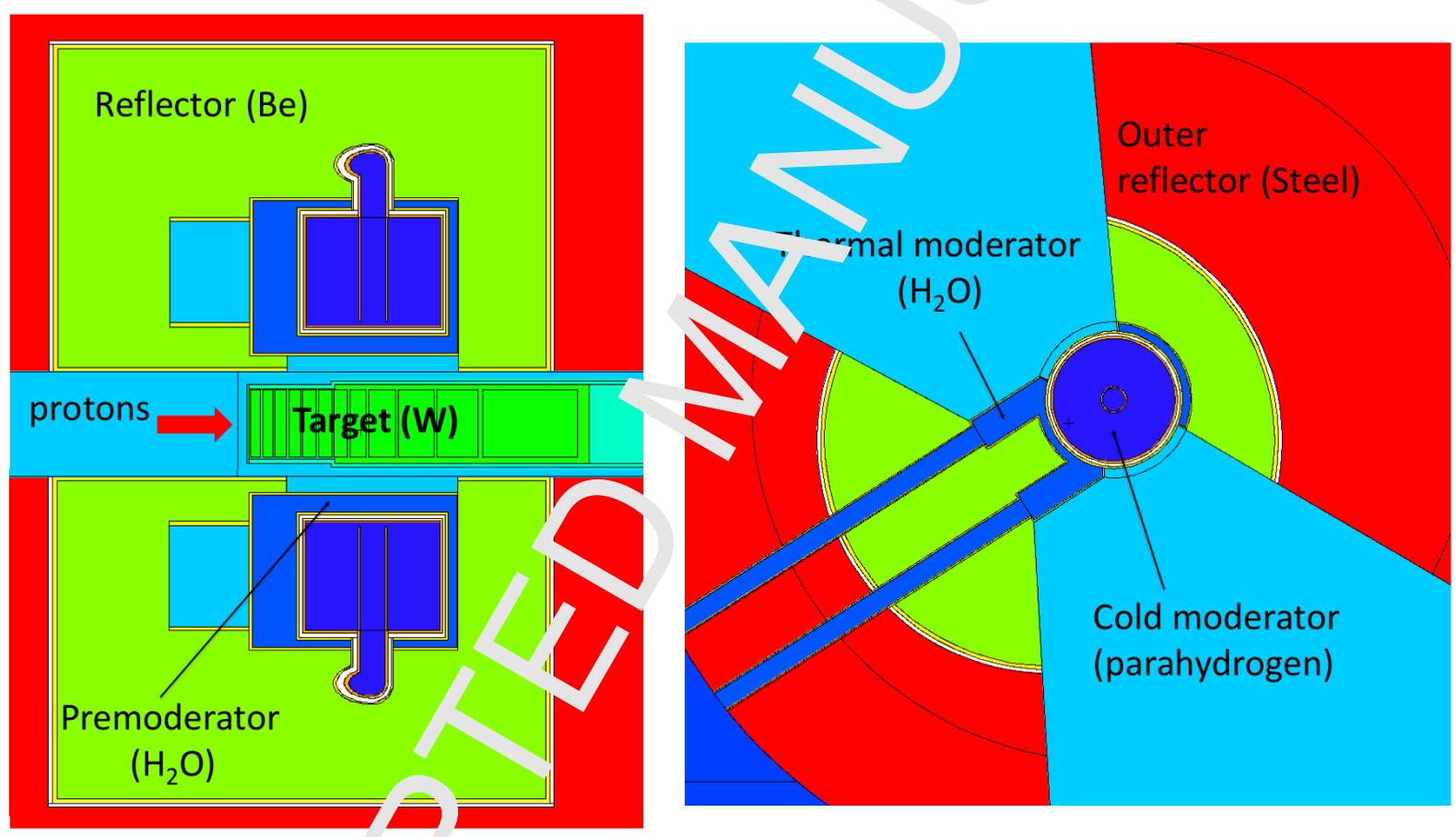
Figure 25. MCNPX models of the two butterfly designs. Top: butterfly 1 design, with a single ${ }^{1} \mathrm{~d}$. roderator vessel and light water moderators, all placed above the neutron production hot spot. Bottom: butterfly 2 des. $\eta$, wh , the cold moderator is split in two parts, and the water moderator occupies a major part of the hot spot. The for $\ldots_{\perp}$ ints, . ie origin of the beamport inserts, are indicated for both designs with red dots.

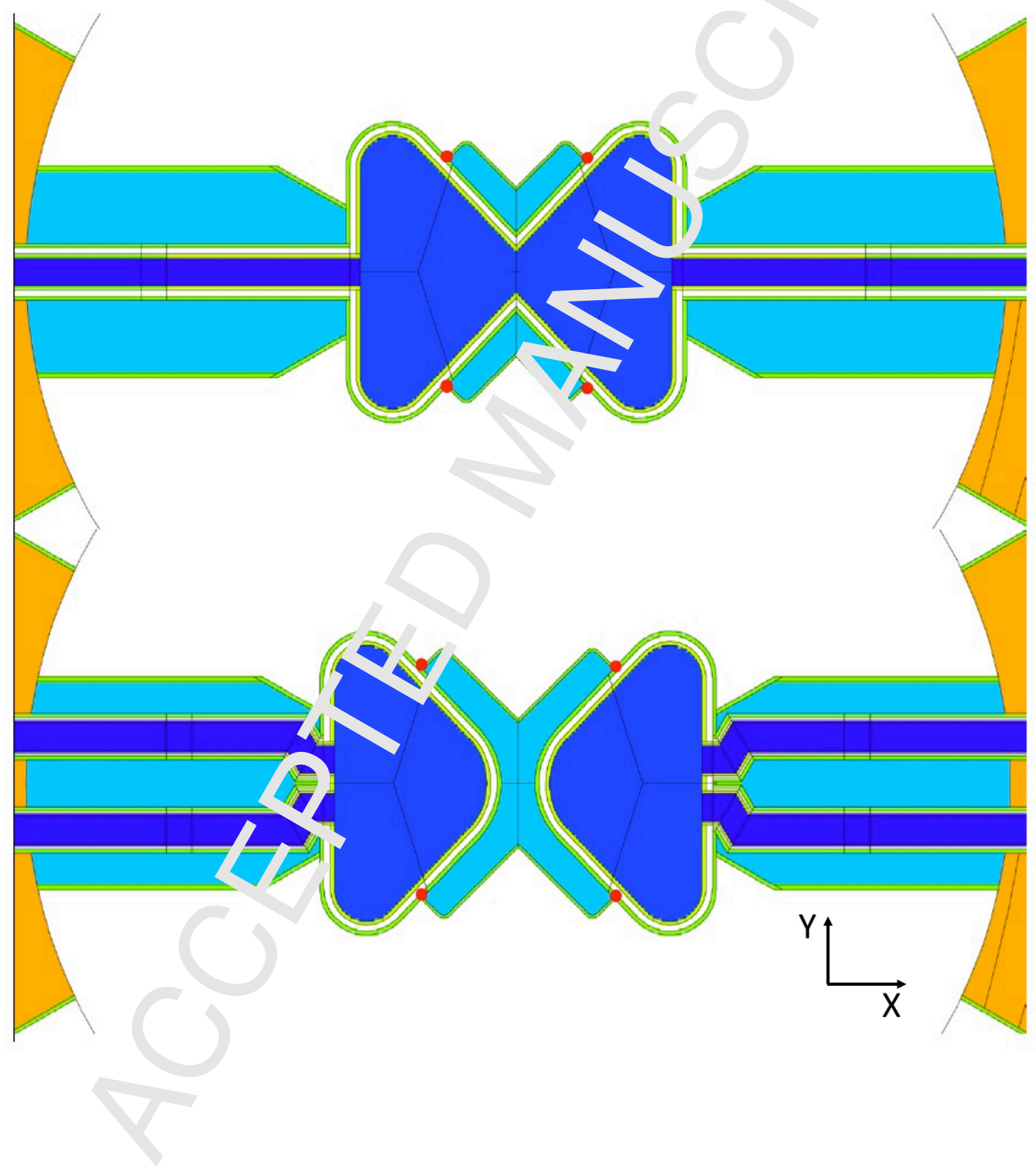


Figure 26. Schematic view of the different beam extraction configurations for thermal (red $a_{\star}$ vws) and cold (blue arrows) neutrons for the pancake and butterfly moderator. For the pancake, near-corner extracti $\Lambda(t, n$ left $)$ and far corner extraction (top right) are possible. For both butterfly concepts, near corner extraction is used (bo . $m$ eft and right). The red dots show the beamline focal points.

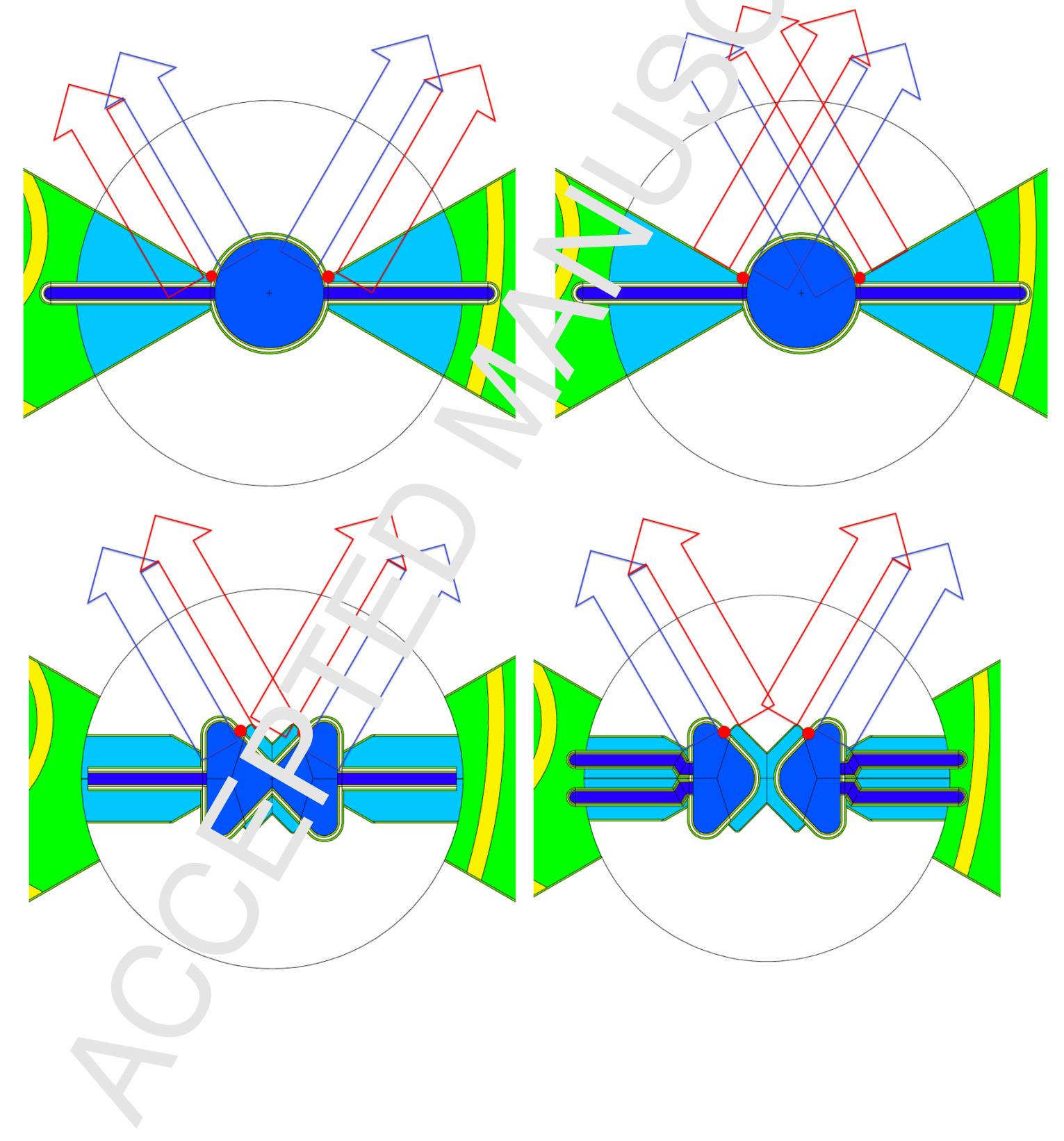


Figure 27. Time-average integrated thermal and cold brightness for the beampor $s$ in the outh and East sectors. Thermal brightness $B_{20-100}$ integrated between $20 \mathrm{meV}$ and $100 \mathrm{meV}$. Cold brightness $R_{n_{-}} \mathrm{v}$, rated between 0 and $20 \mathrm{meV}$. The horizontal viewed width at the moderators is $6 \mathrm{~cm}$.

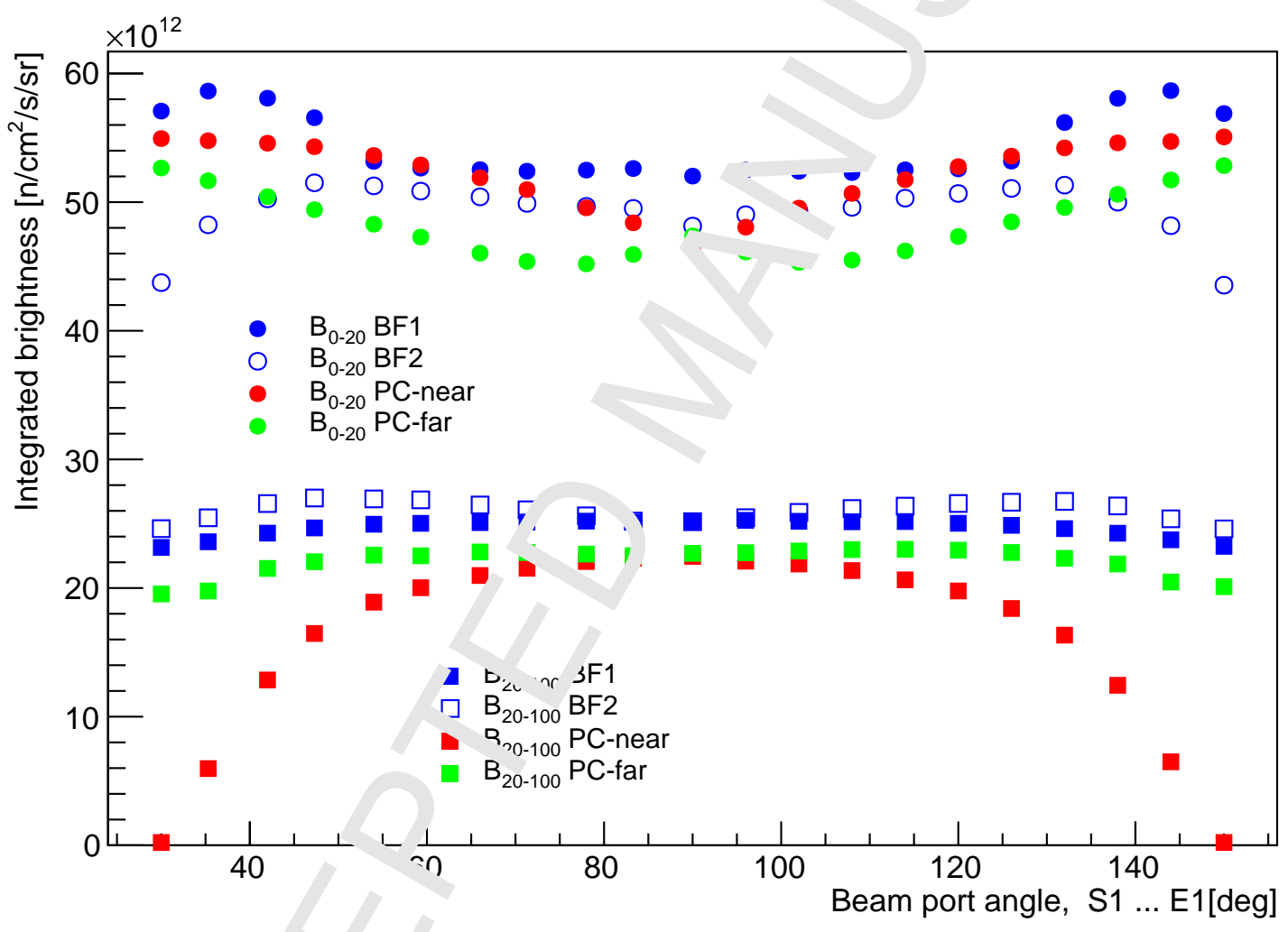


Figure 28. MCNPX geometry used for the brightness calculations of the butterfly 1 mode or, side view. Proton beam comes from the left impinging on the tungsten target. The spallation target contains tung cen bri-ks (green) nominal density of 19.3 $\mathrm{g} / \mathrm{cm}^{3}$, except for the last two rows which contain stainless steel bricks. However some ca! ulations were performed using a single cell with density of $15.1 \mathrm{~g} / \mathrm{cm}^{3}$ to account for the fraction of helium in tr arge wucording to which the filling factor of tungsten is $78 \mathrm{vol} \%$. The water premoderator (light green) between target a: $\mathrm{d} \mathrm{m}$ uerc or has a $8 \%$ volume fraction of $\mathrm{Al}$, to account for flow channels, not modelled. The beryllium reflector (light bluc' includos - ater channels (light green) according to engineering drawings. The outer reflector (orange) is made of stainless steel, wlu. $10 \%$ volume fraction of water, for cooling.

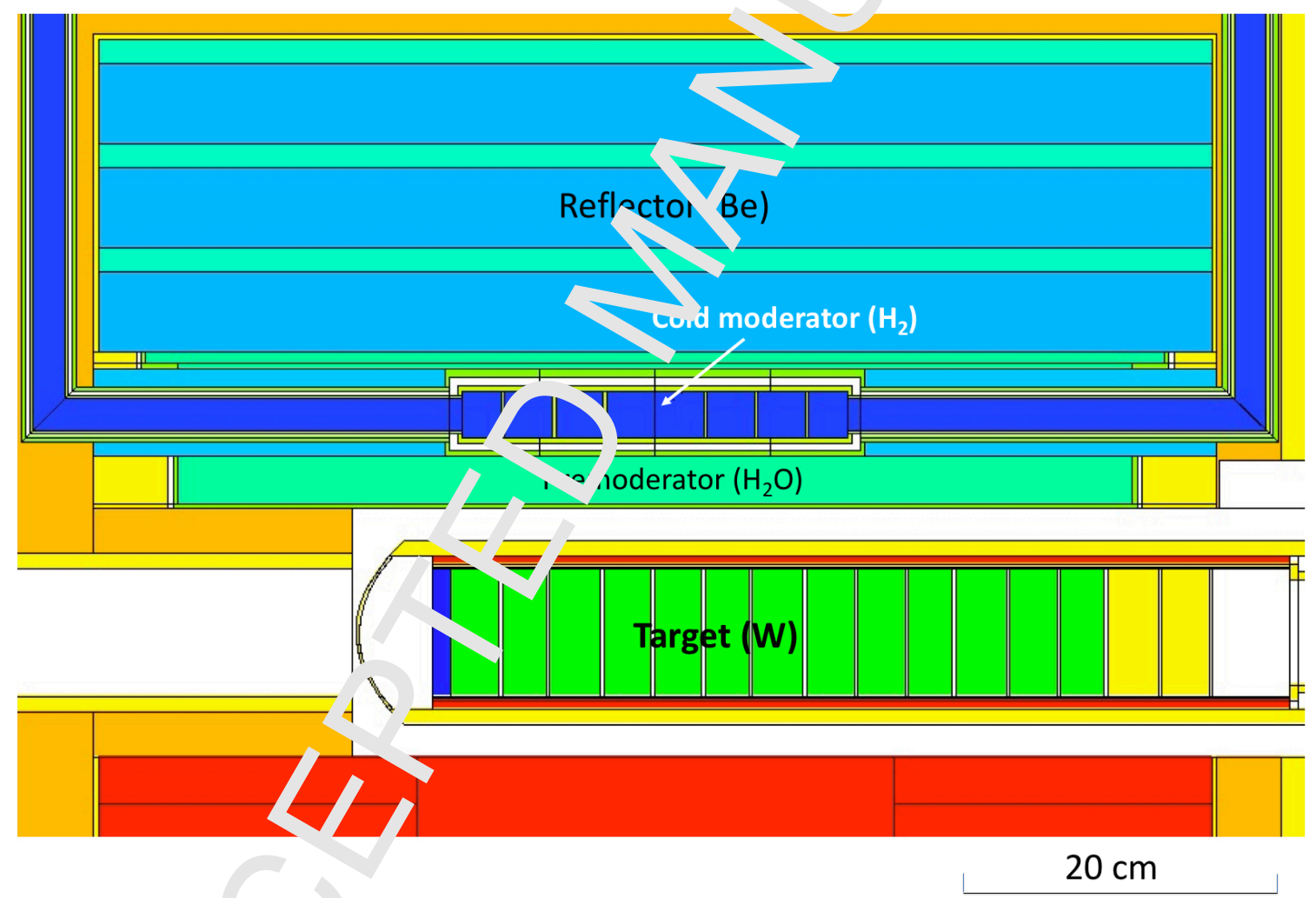


Figure 29. MCNPX geometry used for the brightness calculations of the butterı. $1 \mathrm{~m}$ ' derator, side view showing beam extraction channels. The proton beam is entering the figure in correspondenc to tr entral area of the target.

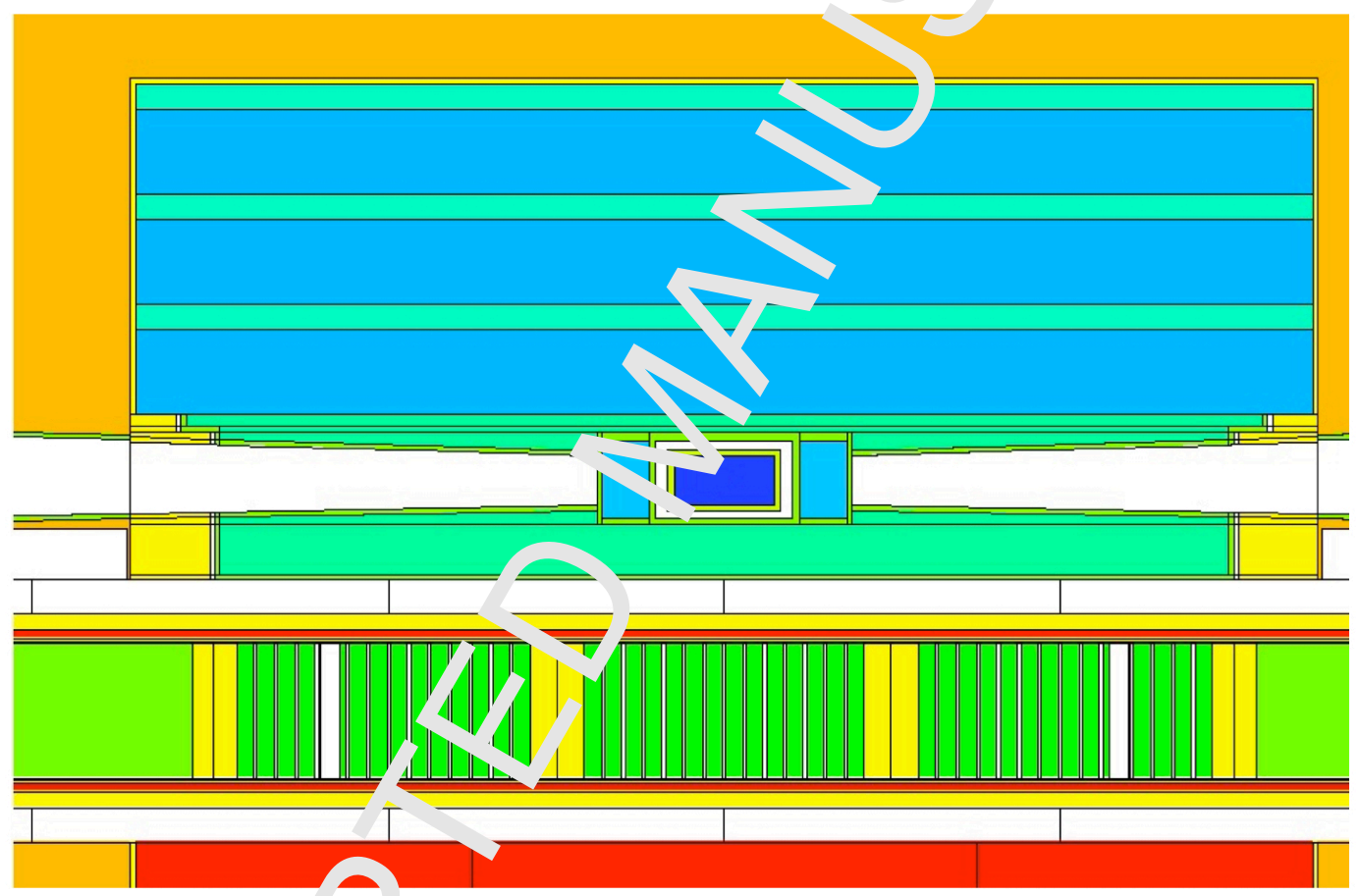




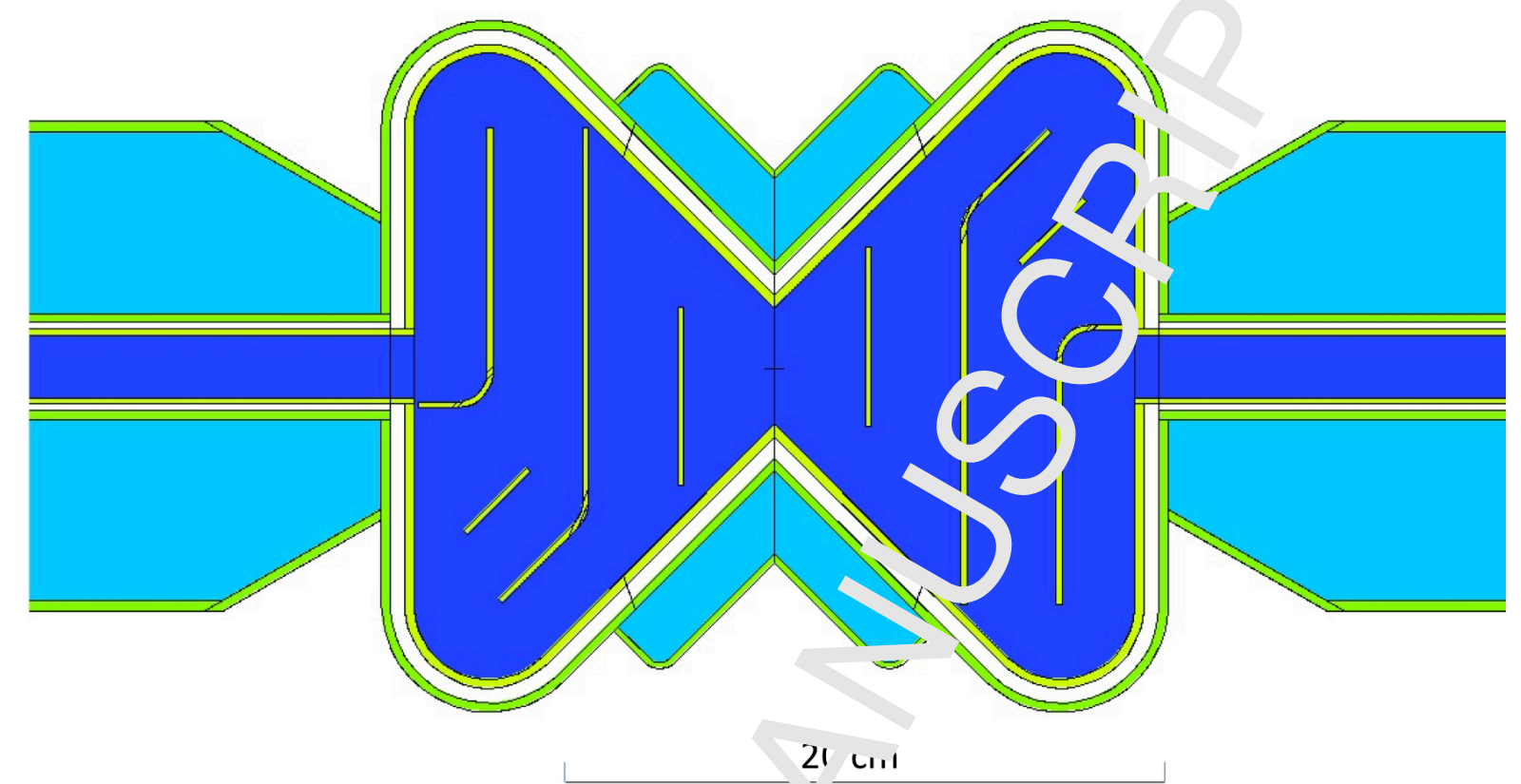

behaviour of the moderator is present. On the other hand, the thermal brightness does not shown a strong dependence on the beamport location.

Figure 35 shows the thermal and $r$ sld pt. 's spectral brightness, averaged over the 42 beamports. The

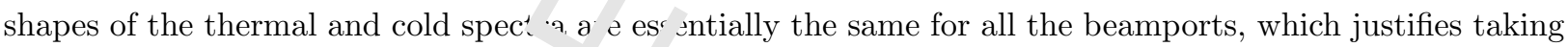
the average.

The distribution of the time aver $a_{c}$, integrated brightness for the butterfly 1 moderator with all the available engineering details 's sh wn in Figure 27.

When optimising the raide 'sign for an individual instrument, a two-stage process is recommended. Firstly, the instruments suid shr ald be oriented within the beamport insert to point at the centre of the relevant source: for $\mathrm{a}^{\mathrm{I}} \mathrm{d}$ ins . ment, that is the centre of the circle defined by the circular arc defining the nearest lobe of the ydroger moderator. For a thermal or bispectral instrument that is the centre of the $V$-shape of the o wide surace of the thermal moderator.

The exact o ientatio 1 should then be refined using the McStas source component to maximise the instrument pe frrmance. The effective brightness of the source will vary by up to about $15 \%$ depending on the width and ' gular acceptance of the guide system.

The performance of the ESS source can be compared with the official ILL brightness values from the 
Yellow Book [4]. The original design goal of ESS was to achieve a cold peak brightness 30 times the average ILL brightness [1]. As shown in Figure 36, with the present design using low-dime ssion ' ' moderators, we are far above this goal. The cold brightness of the butterfly 1 moderator is (at 4 \%) a out 150 times higher than the yellow book value. The thermal brightness at $1.5 \AA$ is about 8 times $\mathrm{n}_{\iota_{c}}$ er than $\operatorname{ILL}^{1}$. Similar ratios are found for the integral values in the ranges considered in this work ( $\mathrm{f}$. ' $\iota_{c}{ }^{\prime} \mathrm{l} e \mathrm{e}$ ).

The brightness results of Figures 35 and 34, and the numbers in this Ser ion ete to the presented design of the moderator-reflector system. The engineering design is ongoing and chi res to the final design will affect the moderator performance. The results are given assuming th presen e of a fast neutron reflector below the target giving an increase of $9 \%$ in neutronic performa -3 or having a second moderator assembly below the target (see Table 1) A l wer performing reflector, or the presence of a bottom moderator similar to the top one (i.e. with water premoderator) would reduce the brightness by some \%. It will be a strategic decision to be +aken $c_{j}$ a later stage, whether to focus on the top moderator or to consider different sources at the botu $\eta$ moderator, which will depend on several factors, including the proposal of novel source concepts. ¿ c priority will be given for many years to a high performing top moderator, it will be worth considerir _ nntimized fast neutron reflector which would give a further increase in performance of the top moderator.

\subsection{Absolute uncertainty}

A question arose during the design phase, of a pussible higher impact of the engineering reality on the neutronic performance of the low-dimens: snal $\mathrm{n}_{\mathrm{L}}$-derator, with respect to a volume moderator, that would decrease the brightness gain in a real situaı $\urcorner$. ${ }^{\top}$ nis was studied in detail, also because before deciding on a single butterfly-1 moderator, detailed eng: .eering for a configuration of two butterfly-2 moderators, one $3 \mathrm{~cm}$ high, the other $6 \mathrm{~cm}$ high, was per ormeu ${ }^{7}$ nis allowed to monitor the impact on the brightness of the two moderators, when engineering $d$ tais rere progressively added to the model. It was found that the relative performance of two moderate $\mathrm{s} \mathrm{n}$ th significantly different heights $(3 \mathrm{~cm}$ and $6 \mathrm{~cm})$ does not change in the transition from physics mode ${ }_{\perp}$ models with added engineering details. This result is consistent with the values from Table 2 that cor pare the updated TDR model to the low-dimensional moderator engineering models.

Although the ful' enginet ing design of the butterfly-1 has not yet been performed, an attempt to include all the engineering $\operatorname{deta}^{\cdot{ }^{\prime}}{ }^{\prime}$.nd associated penalties in neutron production) was made in agreement with the

\footnotetext{
${ }^{1}$ According to a ${ }^{-n n^{+}}$unpublished compilation of experimental and computational data, the ILL brightness should be corrected, so $i$ at $u$ rage the thermal brightness is lower by about a factor of 2 , and the cold brightness is on average higher, also by at at a factor 2, with respect to the official data. However, in this work we stick to the comparison with the official yellow book values.
} 
information available from the existing design. A preliminary design of the flow-channe s was performed [28] and used for the final brightness calculations.

To determine absolute uncertainties on the brightness values one must also ons ler the uncertainties related to the Monte Carlo calculations. MCNPX calculations are based on spallav - 1/evaporation models, and on nuclear libraries (available mostly up to $150 \mathrm{MeV}$ energy), which inclures al the scattering kernels. A detailed study of uncertainties related to these contributions was dor du ing he work for the TDR mdoerator [1]. On the basis of those results, and considering the more mature 'ovel of the present design, we expect an absolute uncertainty of $15 \%$ on the brightness calculatec from $\mathrm{tr} \geqslant$ model.

\section{Summary and Conclusions}

The design of the ESS moderators presented here is the result or 'n extensive work that considered all the relevant aspects to maximize the flux at the sample + the ${ }^{n}$ - ruments. This approach allowed the selection of an optimal, bright source (the moderators), whiln $\mathrm{m}$. ring that all the aspects related to beam extraction configuration, bispectral extraction, and phase-spe ${ }^{\circ}$ transport were taken into account.

The results from the research of the optimal mode at $r$ aesign for ESS has had a profound impact on the configuration of the facility. The main results . his , ork are summarized below.

1) Brightness increase by using low-dimensı nal moderators. The flat moderators adopted for ESS offer a clear brightness increase with respect to the original TDR design based on volume moderators: the cold brightness increases by more thar a in tor of 2.5 with respect to the original design for ESS.

2) Use of a single moderator sys $>\mathbf{m}$. It was realized at an early stage that a single flat moderator would be sufficient, as the differe ce $\boldsymbol{i}_{\text {i }}$ perıormance between a single low-dimensional moderator with $\left(2 \times 120^{\circ}\right)$ openings, and two low-dime . ona' moderators with $\left(2 \times 60^{\circ}\right)$ openings each, would be negligible. This finding opened the possibil , y, sventually adopted, of having an initial instrument suite viewing moderators only above the target ith clear advantages in terms of performance, engineering, operation and upgradeability.

3) Moderator shap for op.imal beam extraction. The chosen butterfly moderator is the best solution we could find to ${ }^{\prime \prime}$ ' ver ' .gh brightness to all instruments since it maximizes both thermal and cold brightness for a largr $\left.\left(2 \times 1^{\wedge}\right)^{\circ}\right)$ opening angle and improves the beam extraction.

4) Upgrade pos ibilit: ss. The chosen configuration, where all initial instruments foreseen for ESS point to the top moder tor, leaves open several attractive possibilities for future use at ESS. Despite having all the instrume. 's in the initial suite viewing the top moderator, the beam extraction system of ESS is designed so . 'au $\because \frac{f}{\mathrm{f}}$ the 42 beamports can view either the top or the bottom moderator, or even both.

With a high 'rightness moderator at the top, the bottom area could be reserved in future for a source of neutrons which should be complementary to the top one. Several possibilities could be explored, such as: 
Figure 31. Projected width at the thermal and cold moderator surface for the different beamports, for re butterfly 1 moderator. See explanation in the text.

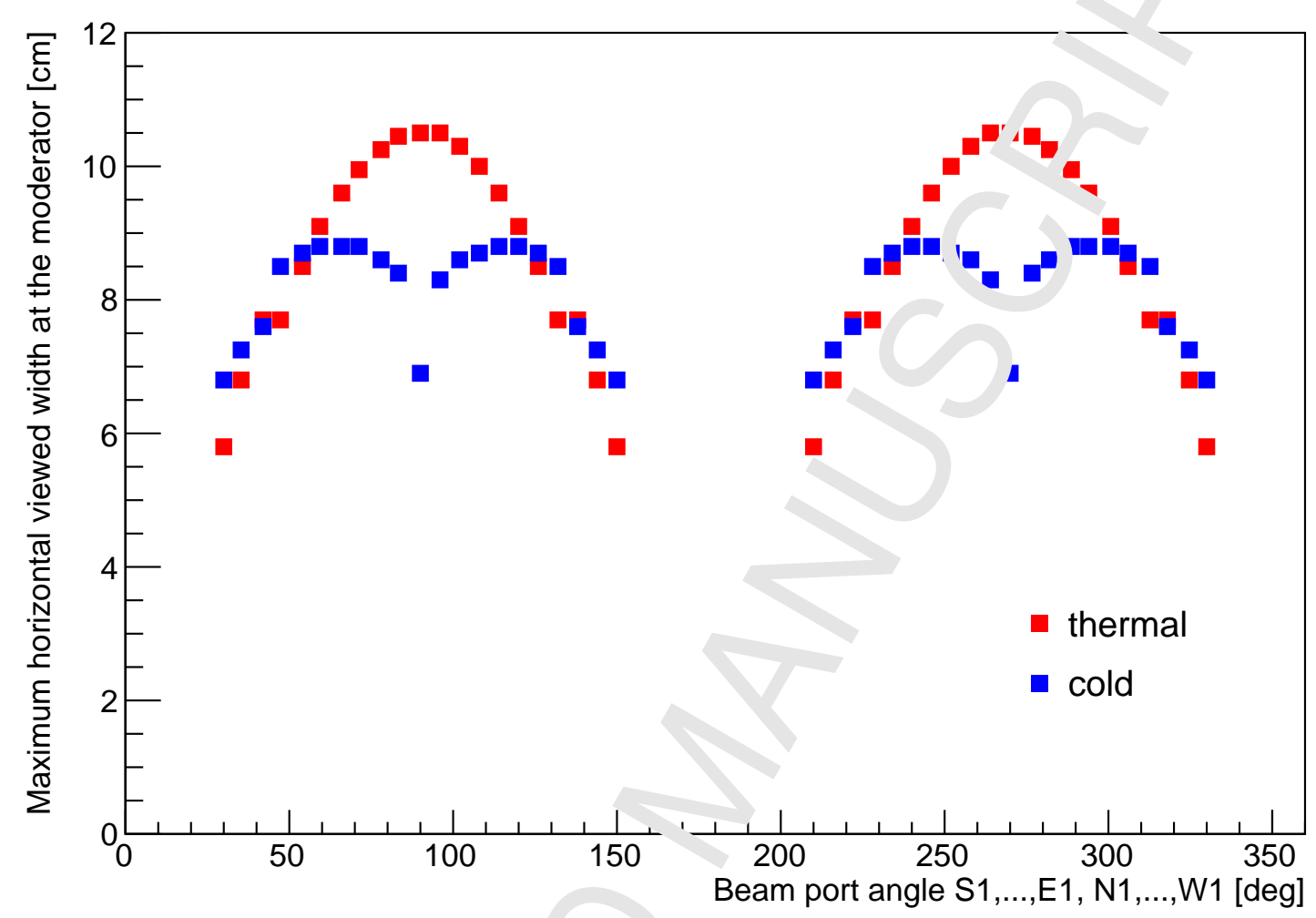

- Very Cold Neutron (VCN) s uru. $\mathrm{T}^{\mathrm{T}}$ ere is increasing interest in VCNs for neutron scattering and fundamental physics applir tu $\cap$ s [22]. The production of neutrons with $\lambda>20 \AA$ requires different materials and temperatr a than for cold neutrons. An interesting technique to increase the flux in this wavelength region nig'.t come by the use of diamond nanoparticle reflectors [31].

- High-intensity mor ${ }^{2}$ rat $, \mathrm{r}, \mathrm{s}^{\prime} \mathrm{ch}$ as a large $\mathrm{D}_{2}$ moderator, for specific experiments like the nnbar beamline $[32,33]$.

- A high-brightn ss mor erator, even surpassing the brightness of the top moderator, which could be achieved $e$ iher $b$ : using tube moderators (good for a few beam lines only, due to their strong directionality), " by $f$ rther reducing the height with respect to the top moderator. Tube moderators are discusi 'u 11 . 'pendix B. 
Figure 32. Brightness distribution along a horizontal $X$ axis perpendicular to the beamport dir ctior shown for W1 and W11 beamports. Dashed lines pass through the focal points and the 0 of the $X$ axis. At $90^{\circ}$ (W11) th old distributions from the two sides of the cold moderator have the same intensity, as expected.

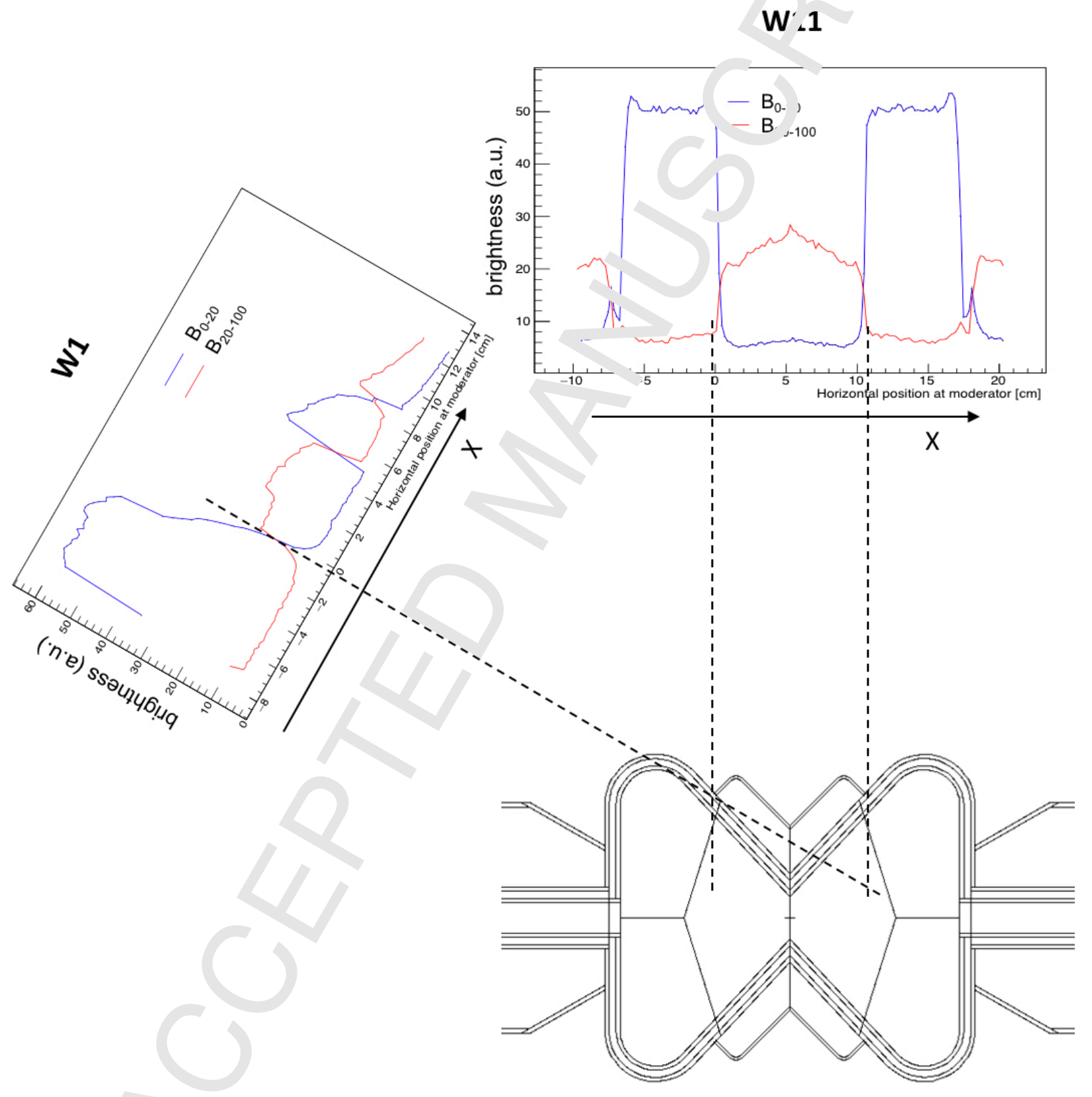


Figure 33. Horizontal brightness $B_{0-20}$ and $B_{20-100}$ distribution for the W3 be mport. The $X$ axis corresponds to a line perpendicular to the beamport axis, with the 0 corresponding to the focal point ( $\mathrm{s}$ - Figurf 32). These distributions are also valid for the S3, E3 and N3 beamports.

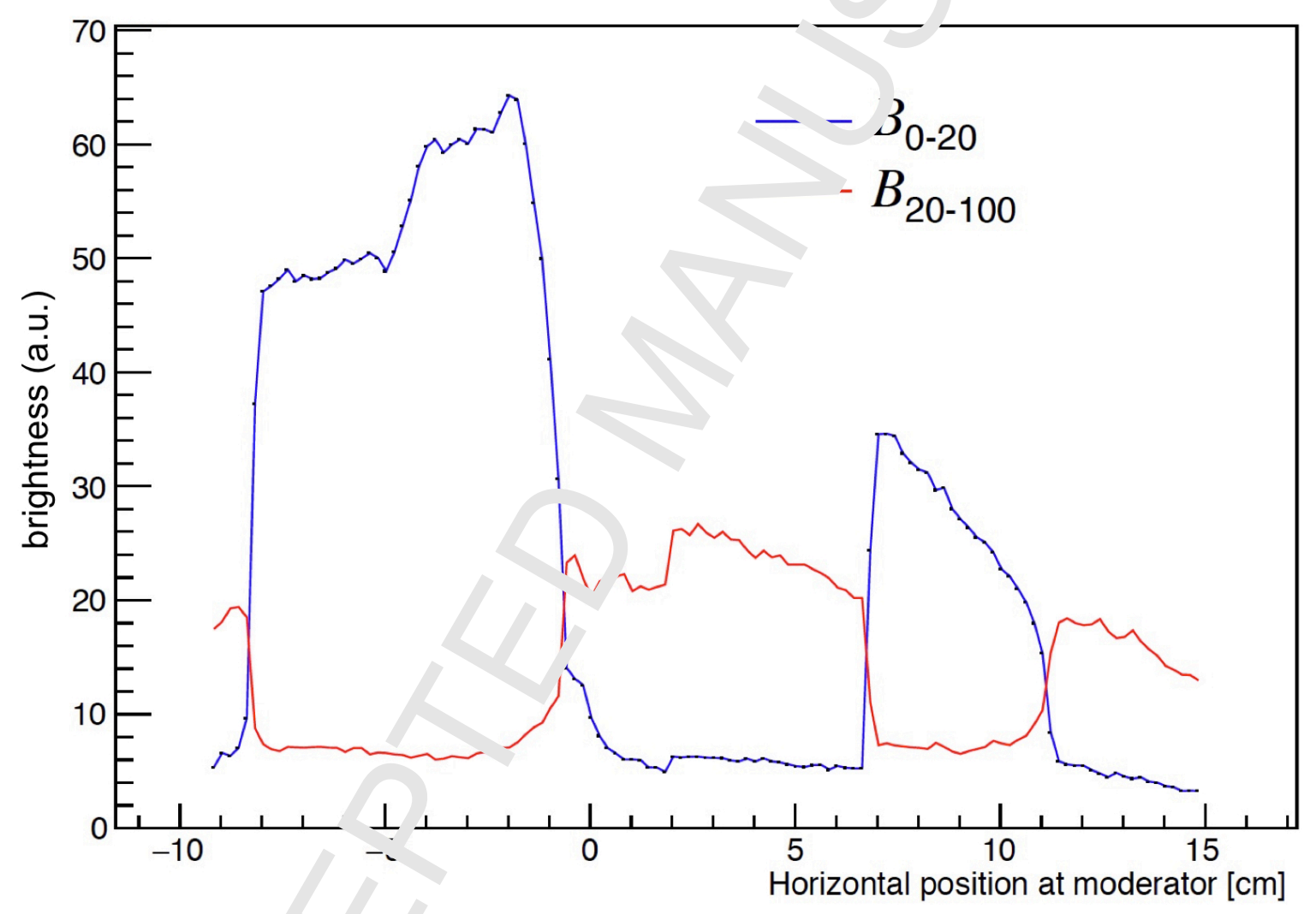




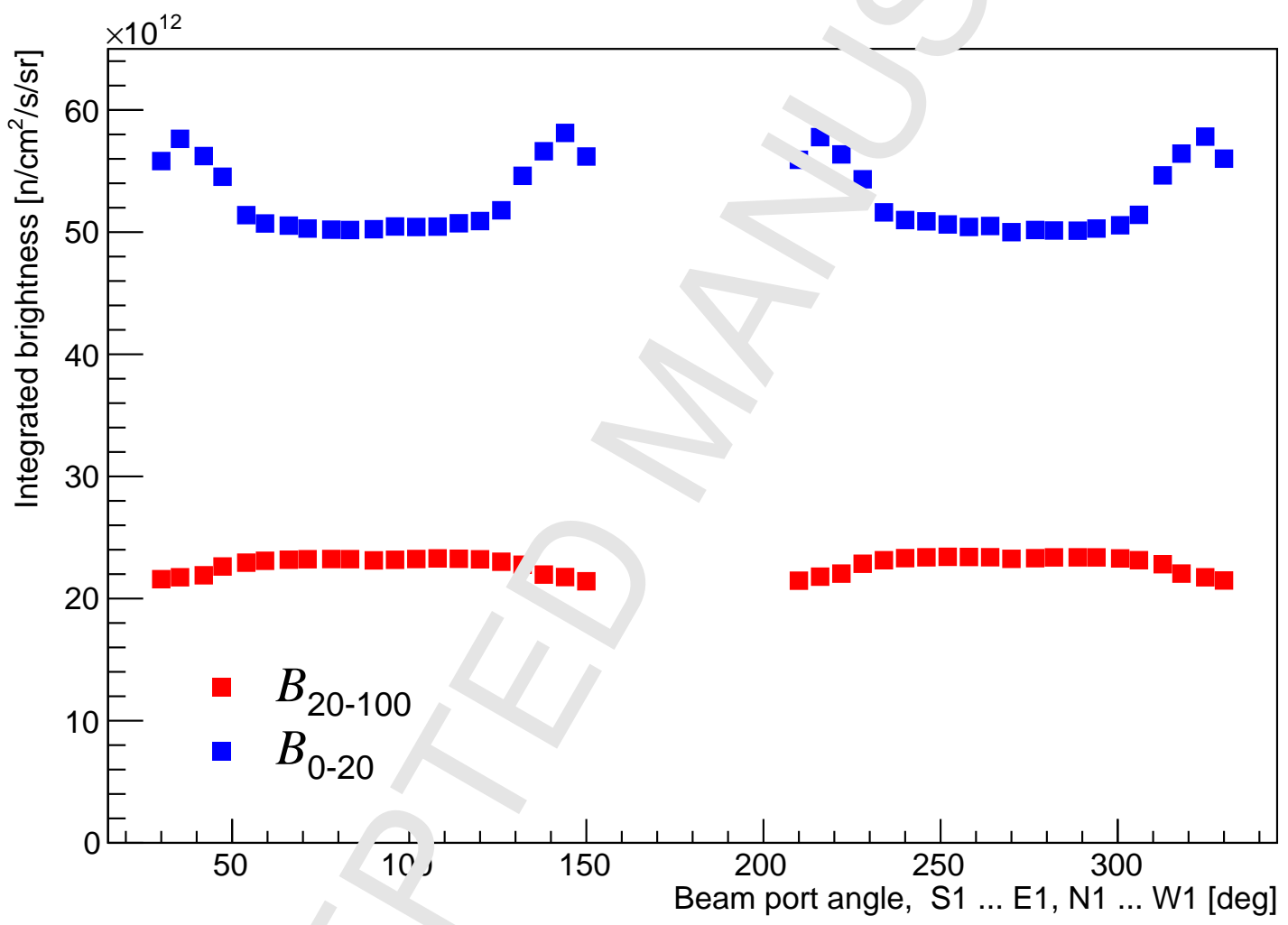


Figure 35. Thermal and cold peak wavelength spectral brightness. Spectra are al raged $c$ er the viewed moderator surface area of $3 \times 6 \mathrm{~cm}^{2}$.

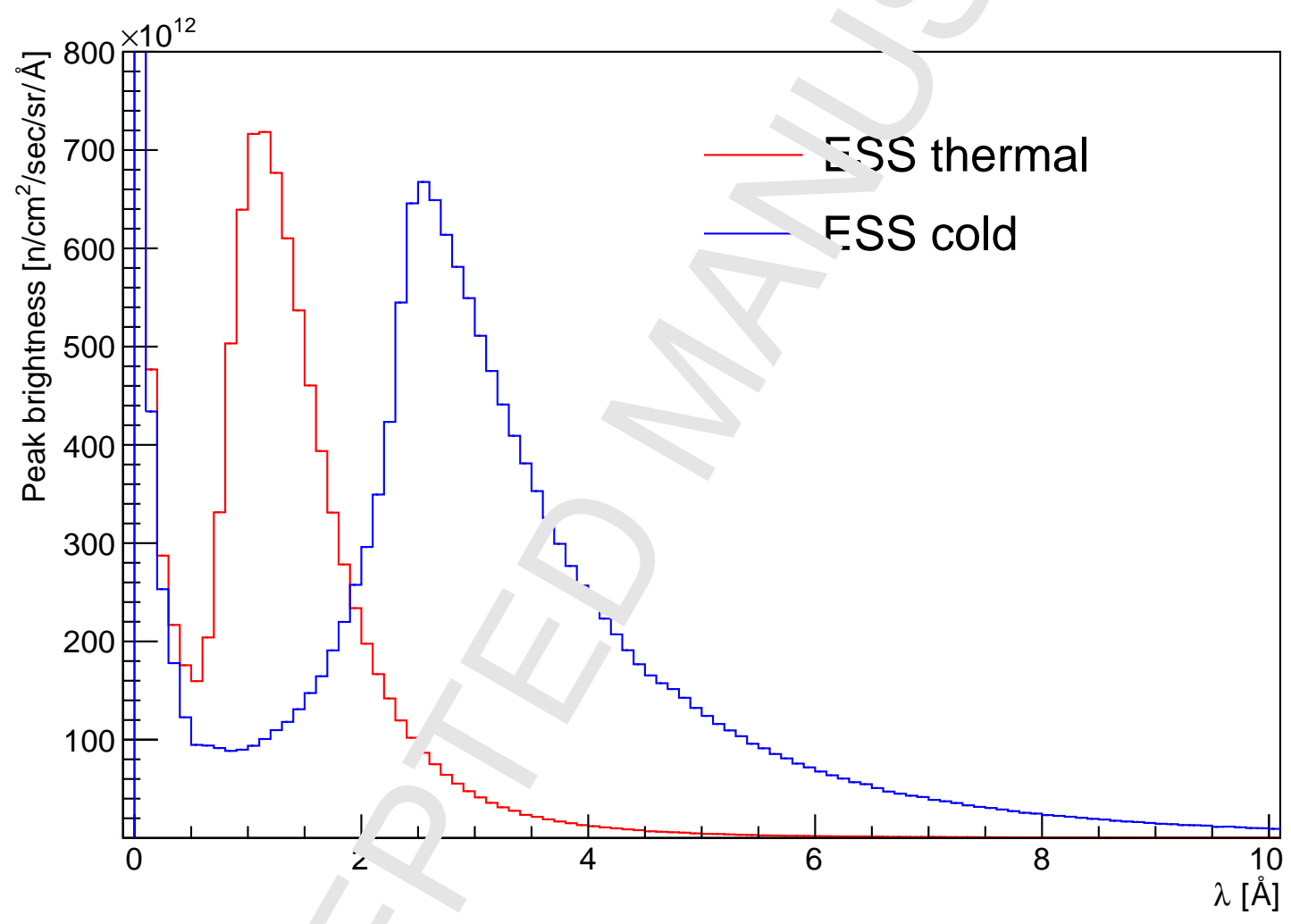


Figure 36. Brightness spectra averaged over 42 beamports for $3 \mathrm{~cm}$ high moderator, am ared with ILL official curves [4].

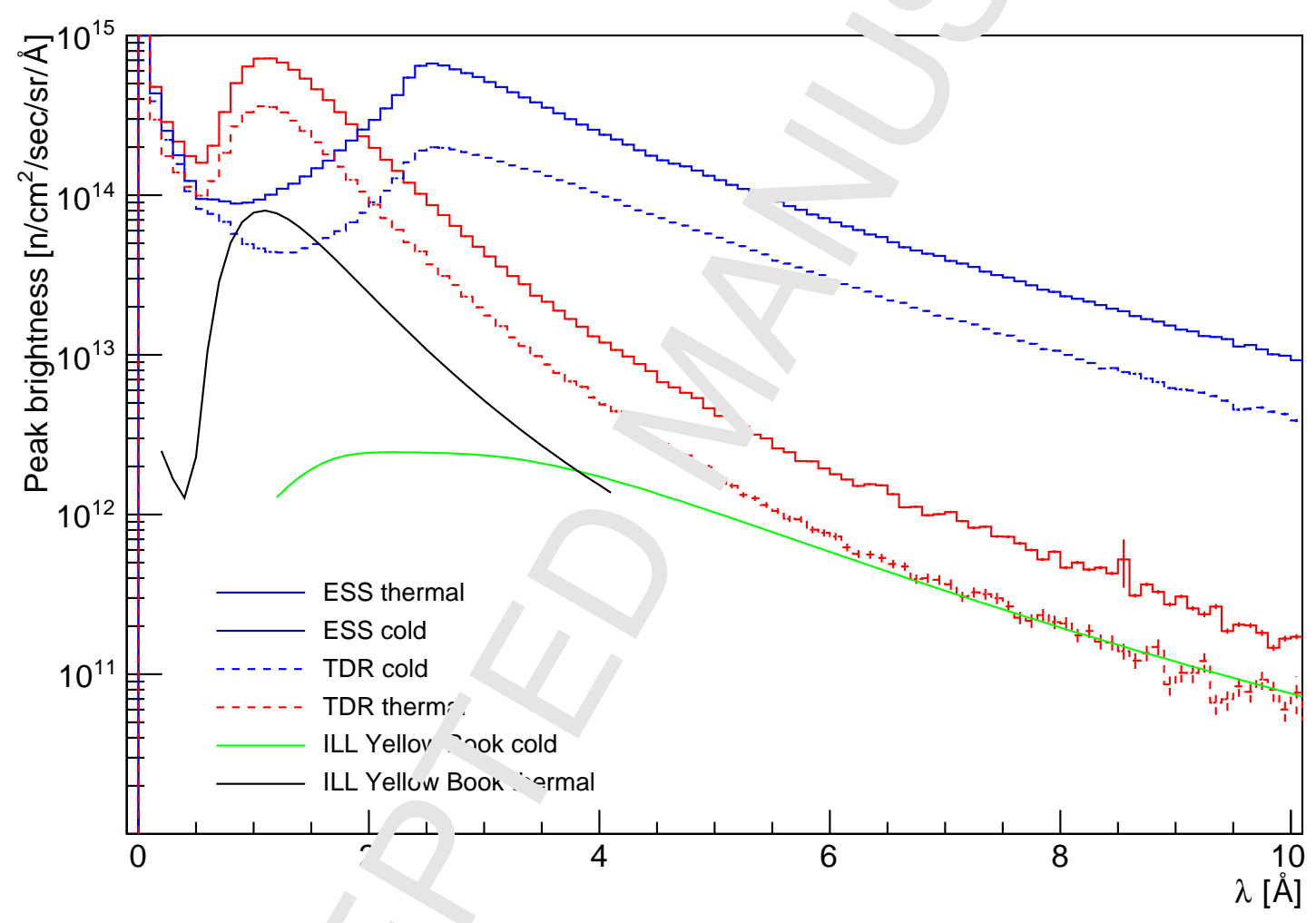


Figure 37. Cold collimators used in the MCNPX model for the brightness calculation. In he figu the collimators for the S1, S5 and S10 beamports are shown. The viewed width at the moderator is of $6 \mathrm{~cm}$, th ${ }^{\text {' }}$ ight . of $3 \mathrm{~cm}$. The collimators intersect the point shown with the yellow dot, which is placed at $1.1 \mathrm{~cm}$ from the focal point (rt iot), the origin of the axes of the beamports.

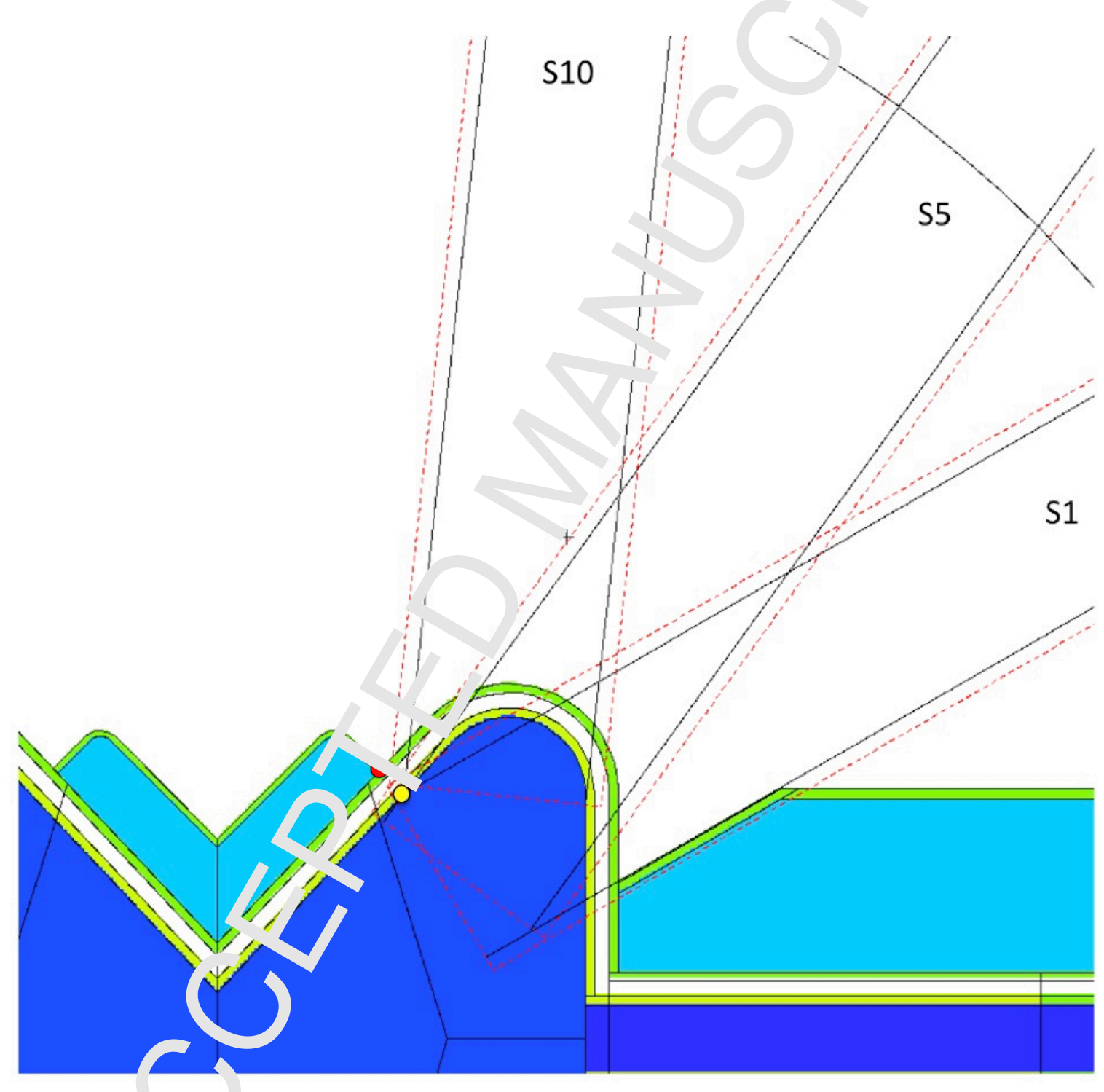


Figure 38. Geometry of box moderator.
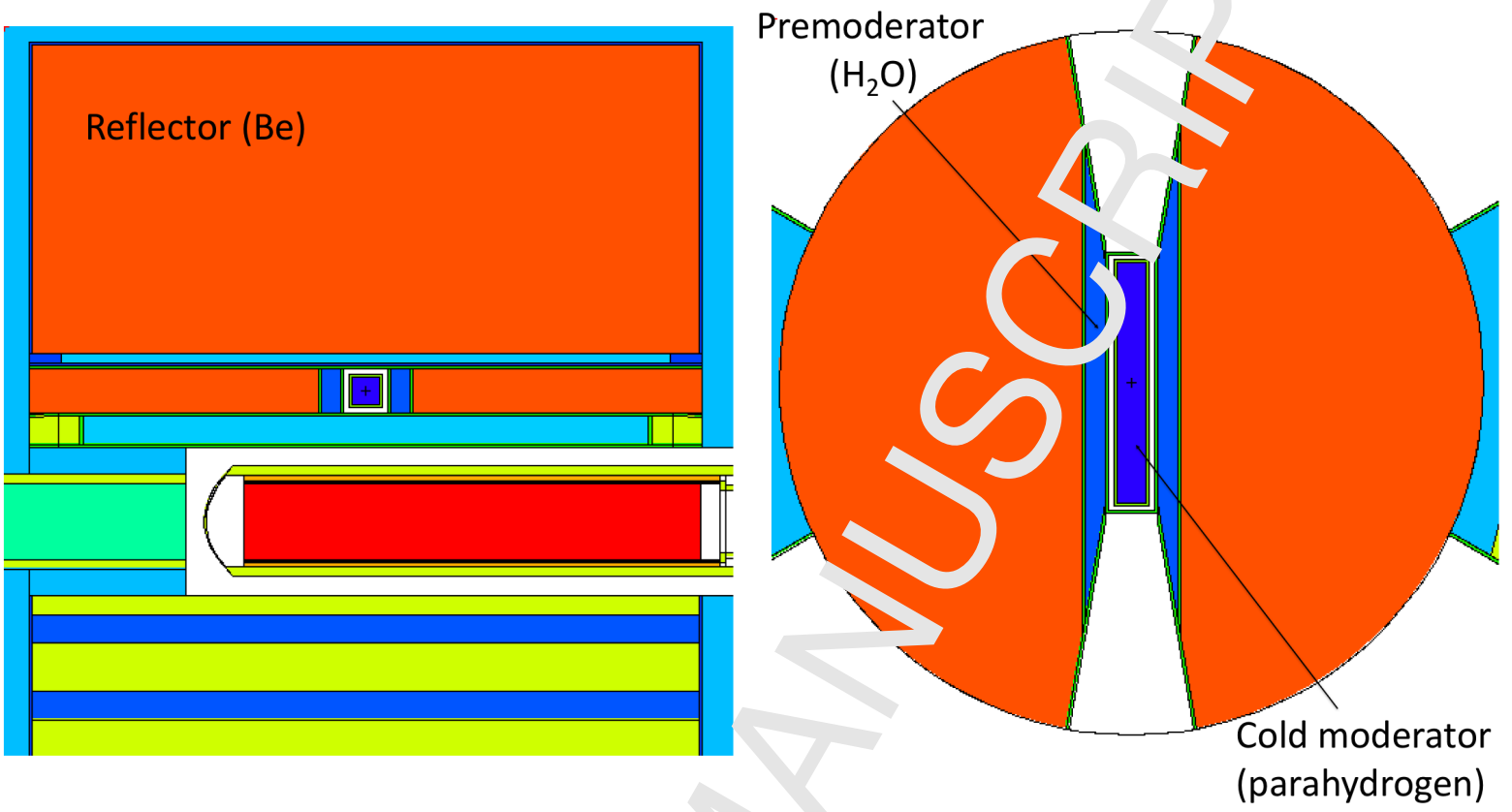

Figure 39. Brightness map as a finct 1 - of teral dimensions of a box moderator of $24 \mathrm{~cm}$ length.

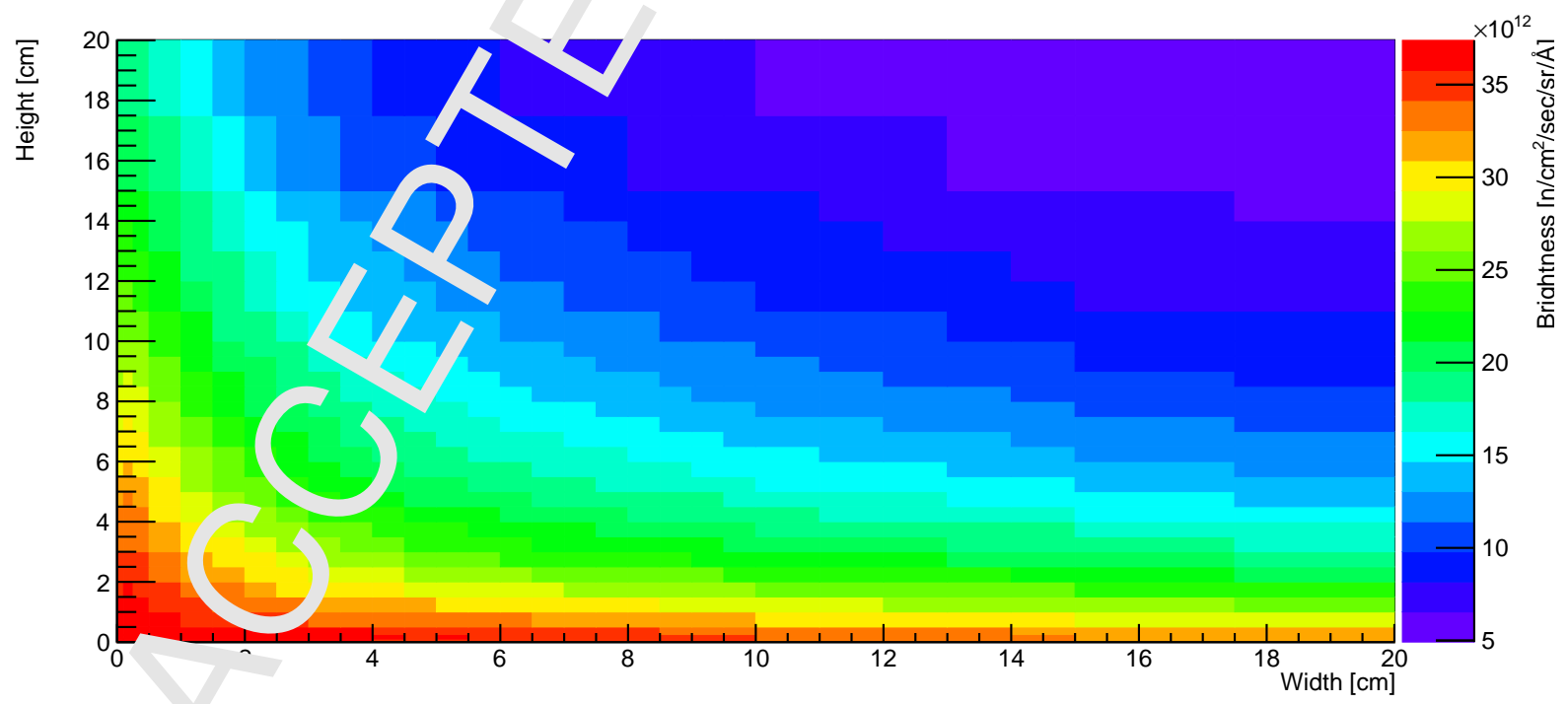




\section{AppendixA. Brightness and figure of merit}

\section{AppendixA.1. Brightness and Liouville's theorem}

We first show the link between the brightness of a source and Liouville's theoi $\eta$, nd, as a consequence, the fact that brightness is a constant of motion.

We define as neutron angular density $N(\mathbf{r}, \boldsymbol{\Omega}, E, t)$ the number of ne' cror $\sim n$ at the position $\mathbf{r}$ with direction $\Omega$ and energy $E$ at time t, per unit volume, per unit solid anglt, ser unit energy. The vector position $\mathbf{r}$ can be at the surface of a moderator, while $\Omega$ is the directic a of $n \in$ tron emission:

$$
N(\mathbf{r}, \boldsymbol{\Omega}, E, t)=\frac{n\left(\mathbf{r}, \boldsymbol{\Omega}, E, t^{\prime}\right.}{d V d \Omega d E}
$$

The unit of $N$ is $\mathrm{n} / \mathrm{cm}^{3} / \mathrm{sr} / \mathrm{eV}$.

The brightness $B$ is the product of the speed $v$, times the sutron angular density.

$$
B(\mathbf{r}, \boldsymbol{\Omega}, E, t)=v N\left(\mathbf{r}, \boldsymbol{\Omega},\left\llcorner,{ }^{\iota}\right)=v \frac{\cdots(\mathbf{r}, \boldsymbol{\Omega}, E, t)}{d V d \Omega d E}\right.
$$

The units of the brightness is $\mathrm{n} / \mathrm{cm}^{2} / \mathrm{s} / \mathrm{sr} / \mathrm{eV}$.

On the other hand, the phase space density $\rho(\mathbf{q}, t)$ is the number of particles in the phase space volume $d \mathbf{q} d \mathbf{p}=d V p^{2} d p d \Omega$ at time t:

$$
\rho(\mathbf{q}, \mathbf{p}, t)=\frac{n(\mathbf{r}, \boldsymbol{\Omega}, E, t)}{a \mathbf{v}^{2} d p d \Omega}=\frac{v}{p^{2}} \frac{n(\mathbf{r}, \boldsymbol{\Omega}, E, t)}{d V d \Omega d E}
$$

where we used the relationship betweeı '-inet c energy and momentum $E=\frac{p^{2}}{2 m}$.

Liouville's theorem states that th' phr e sface density $\rho(\mathbf{q}, \mathbf{p}, t)$, i.e. the number of particles in the phase

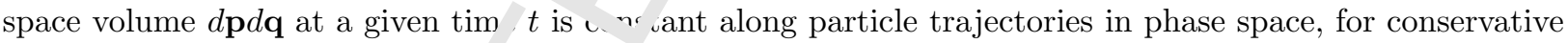
force fields.

From the above two equat on it follows that, for constant momentum, the neutron angular density and the brightness $B$ are constanı ' , ng the direction of motion.

These results are alsc vali if brightness is expressed as a function of the $\lambda$, as it is commonly expressed, instead of $E$.

In practical term, conse vation of the phase space density along the neutron trajectory (in phase space) means that knowing $t_{2}-h$ ghtness, it is possible to determine the absolute flux at any point of the beam extraction systc n. In 1 ality, beam losses must also be considered. Neutrons emitted in a volume $d V$ in direction $d \Omega$ aroun. ${ }^{\prime}$ will be transported in a guide system, assuming only elastic scattering collisions (with no change in $1 \mathrm{u}^{\prime}$ 'on speed) and as the phase space density is constant, the divergence of the neutrons at the sample will depe. $\mathrm{d}$ on how focused is the beam (the more focused is the beam, the higher its divergence). 
AppendixA.2. Figure of merit

To calculate the brightness with MCNPX we use f5 point detector tallies that calcuı ' 5 the flux at a point [8]:

$$
\Phi(\mathbf{r})=\int d E \int d t \int d \Omega v N(\mathbf{r}, \boldsymbol{\Omega}, E, t)
$$

Thus, the f5 calculation integrates the brightness over $4 \pi$ sr. If the in七 ${ }{ }$ „tion is done in a solid angle $\Delta \Omega$, defined from a point at a distance $r$ much greater than the mode ator d'nensions, that subtends only the moderator emission surface, so that the solid angle is $\Delta \Omega=\Delta S,{ }^{2}{ }^{2}$, th $n$ the brightness defined by MCNPX is

$$
B(\mathbf{r})=\frac{\Phi(\mathbf{r})}{\Delta \Omega}
$$

In practice, $B$ can be calculated with specified bins in en nogy and time, so that, for small solid angles, $B$ is a function of $(\mathbf{r}, \boldsymbol{\Omega}, E, t)$.

In this work we have often used the time-average, $e^{-n n \sigma^{\circ}}$-intugrated brightness between two energy values $E_{1}$ and $E_{2}$ defined as

$$
B_{E_{1}-E_{2}}(r, \Omega)-{\underset{E_{1}}{j_{j}} a_{L}}_{E_{2}}^{\infty} d t B(\mathbf{r}, \Omega, E, t)
$$

\section{AppendixA.3. Tallies}

Brightness is calculated for all the seampos , in the two $120^{\circ}$ beam extraction openings surrounding the moderators, using point detector talı * lacr $d$ at $10 \mathrm{~m}$ from the moderator surface. The tallies are placed at the 42 angles corresponding to 'he actual beamport grid.

\section{AppendixA.4. Collimators}

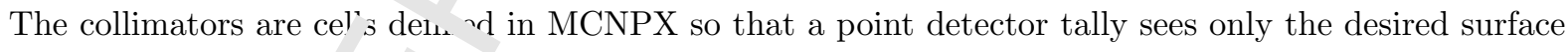
of the moderator (usual' $6 \mathrm{~m}$ w de, $3 \mathrm{~cm}$ high).

Figure 37 shows $t^{1}$. view horizontal widths at the cold moderator used in the MCNPX model for brightness calculatic is. The riewed widths are defined by the solid lines, for all the beamports (in the figure as examples the ${ }^{\prime}$ cumporus $\mathrm{S} 1, \mathrm{~S} 5$ and $\mathrm{S} 10$ are shown). In all the calculations the vertical range seen at the moderator surfa e is alw ys of $3 \mathrm{~cm}$, while the reference horizontal width was of $6 \mathrm{~cm}$. Due to the constraints from the be $m$ oxtraction system discussed in Section 4, there is limited margin in the positioning of the collimators. A stance of about $1 \mathrm{~cm}$ from the focal point is allowed, and in the calculations the collimators were at $1.1 \mathrm{~cm}$ from the focal point (see Figure 37 ). 


\section{AppendixB. Tube moderators}

Based on fundamental physics properties discussed in Section 2.1, the natur ${ }^{-1}$ extensıon of quasi-2dimensional flat moderators are quasi-1-dimensional "tube-like" moderators. ph sics model of a tube moderator adapted to the ESS target-moderator-reflector layout is shown iı Figuı 38 . To study the performance of such moderators we consider for convenience a box shape 1 jic is a basic design which features a box-shaped parahydrogen moderator, surrounded by a premou " tor and beryllium reflector. The geometry of the target and reflector is the same as in the other s, udies, allowing a direct comparison of the brightness. After an optimization of the premoderator thicknes the ptimal length of a reference moderator with $3 \times 3 \mathrm{~cm}^{2}$ cross section was determined to be of ' $4 \mathrm{~cm}$

Then, with a fixed length of $24 \mathrm{~cm}$, a two-dimensional brightness man is a function of width and height, size of emission surface given by (height $\times$ width), was calculated (Fis re 39). The brightness map indicates a strong increase in brightness with decrease in the moder ${ }^{+}$or ${ }_{1}{ }^{*}{ }^{-} \cdot t$, as it has been already observed for the flat moderators. Increase in brightness with decrease in the oderator width is less pronounced. This is due to the size of the neutron production hotspot (cf. Figu $~ 21$ ). The immediate conclusion is that tube moderators would be particularly suitable for compact 'er ron sources where the size of neutron production region is comparatively small.

The stand-alone tube moderator of $3 \times 3 \times \neg 4 \mathrm{~cm}^{3}$ exhibits cold $B_{0-5}$ brightness about 2 times higher than the flat butterfly moderator. However, the rbe moderator is highly directional and it can serve narrow extraction angles only. One way to ar. ase the extraction angles would be to combine several tubes in different configurations.

Other examples of tube-like mode ator ${ }^{3}$, applied to small sources, are given in [34].

[1] S. Peggs editor, ESS + - hni 1 l Design Report, ISBN 978-91-980173-2-8, 2013, http://europeanspallationsource.s , mientific-technical-documentation

[2] F. Mezei, Long pulse spallation sources, \& ysica B 234-236 (1997) 1227.

[3] M. Magán et al., Neutronic c ıaly: s of the bispectral moderator such as that proposed for ESS, Nucl. Instrum. Methods A729 (2013) 417425

[4] Institut Laue-Langevin. ' LL Yellow Book 2008.' http://www.ill.eu/?id=1379, 2008.

[5] K. Batkov, A. Takibay - I. Zar ni and F. Mezei, Unperturbed moderator brightness in pulsed neutron sources, Nuclear Instruments ar "Metho in Physics Research Section A: Accelerators, Spectrometers, Detectors and Associated Equipment 729 (26 3) 500 - i05. doi:http://dx.doi.org/10.1016/j.nima.2013.07.031.

[6] F. Mezei, L. Zanini, 1 T. sibayev, K. Batkov, E. Klinkby, E. Pitcher, and T. Schönfeldt, Low dimensional neutron moderators fc enhan 'd source brightness, Journal of Neutron Research 17 (2014) 101 - 105.

[7] Waters L. S.e $e_{\imath}$ l. 2007 The MCNPX Monte Carlo radiation transport code. AIP Conf.Proc. 896, 81-90.

[8] MCNP, f roneral Monte Carlo N-Particle Transport code, LA-UR-03-1987, 2003.

[9] D. Pelowit‘ er tor, MCNPX Users Manual, Version 2.7.0, Number LA-CP-11-0438, 2011.

[10] L. Zanini, K. \& tkov, E. Klinkby, F. Mezei, E. Pitcher, T. Schönfeldt, A. Takibayev Moderator Configuration Options for ESS, proceedings of the ICANS-XXI conference, JAEA-Conf 20-15-002, 2014 
[11] F. X. Gallmeier, W. Lu, B. W. Riemer, J. K. Zhao, K. W. Herwig, and J. L. Robertson, Concr stual moderator studies for the Spallation Neutron Source short-pulse second target station, Review of Scientific Instr me m 87,063304 (2016).

[12] U. Rücker, T. Cronert, J. Voigt et al., Eur. Phys. J. Plus (2016) 131: 19. https://doi.org/10 -10/epjp/12016-16019-5.

[13] K. H. Andersen, M. Bertelsen, L. Zanini, E. B. Klinkby, T. Schönfeldt, P. M. Bentley : Id J Jaroun, Optimisation of moderators and beam extraction at ESS, JAC 51, 264 (2018).

[14] N. Watanabe, Neutronics of pulsed spallation neutron source, Rep. Prog. Phys. 66 (2nก3) 30. ' 91 , and references therein.

[15] K. B. Grammer et al., Measurement of the Scattering Cross Section of Slow Nr itron L Liquid Parahydrogen from Neutron Transmission Phys. Rev. B 91, 180301(R) (2015).

[16] J. Schwinger and E. Teller, The Scattering of Neutrons by Ortho- and Parahy iogen, Phys. Rev. 52, 286 (1937).

[17] P. Rock, Chemical thermodynamics; principles and applications (Macmillan I 169). ISB J 1-891389-32-7

[18] T. Kai, M. Harada, M. Teshigawara, N. Watanabe, Y. Ikeda, Coupled hyd yen ... _rator optimisation with ortho/para hydrogen ratio, Nuclear Instrum. Methods in Physics Research A 523, 38 (2r 4 ).

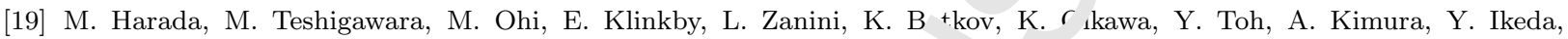
Experimental validation of the brightness distribution on the surfaces of a vled and decoupled moderators composed of 99.8\% parahydrogen at the J-PARC pulsed spallation neutron sour. Volum 903, p. 38, 2018.

[20] R. Golub, K. Bning, and H. Weber, Ultra-Cold Neutrons at the SN $N_{\mathrm{c}}$ Gallation Source, TUM-E21/SNQ-UCN/81-4, 1981.

[21] E. Klinkby, K. Batkov, F. Mezei, E. Pitcher, T. Schönfeldt, ‘ _mancayev, and L. Zanini, In-Pile ${ }^{4}$ He Source for UCN Production at the ESS, Adv. High Energy Phys., 2014, 2014, p. ¿4 ‘39, doi 10.1155/2014/241639

[22] Micklich B.J. and Carpenter J.M (editors), Proceedings oi the $v$ _ hop on Applications of a Very Cold Neutron Source, August 21-24, 2005, Argonne, Illinois, ANL-05/42, Ar $\cdots n n e ~ \perp$ tional Laboratory ( 2005).

[23] L. Zanini, K. Batkov, F. Mezei, A. Takibayev, E. Kı 'kь。 1. Schönfeldt, The neutron moderators at the European Spallation Source, proceedings of the AccApp'15 canonce http://accapp15.org/wp-content/data/index.html, 2015.

[24] T. Schönfeldt, Advanced Neutron Moderators, PhD the. - Technical University of Denmark, 2017.

[25] L. Zanini, K. Batkov, E. Klinkby, F. Mezei, T Crhönfeldt, and A. Takibayev, The neutron moderators for the European

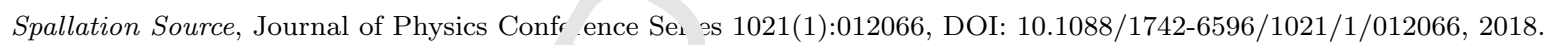

[26] Carpenter, Loong, Elements of slow neutron _ vtter . $g, 2016$.

[27] R. Garoby et al., The European Spallo on ' Jurce Design, Physica Scripta, Vol. 93, N. 1, 2017.

[28] Y.Bessler, private communication, 2010

[29] P. Willendrup, E. Farhi and K. Lef ann, Mcsuas 1.7 a new version of the flexible Monte Carlo neutron scattering package, Physica B, 350 (2004) E735.

[30] T. Kittelmann, E. Klinkby, F B. Knudsen, P. Willendrup, X. X. Cai, and K. Kanaki, Kalliopi, Monte Carlo Particle Lists: MCPL, Comput. Phy. Co imun., 218, 17, 2017, ARXIV:1609.02792.

[31] V.V. Nesvizhevsky, E.V.' ychagin, 'YYu. Muzychka, A.V. Strelkov, G. Pignol, K.V. Protasov, The reflection of very cold neutrons from diamon' now er nr uoparticles Nuclear Instrum. Methods Physics Research A 595 (2008) 631.

[32] E. B. Klinkby, K. Batkov, - T ezei, T. Schönfeldt, A. Takibayev, L. Zanini, Voluminous D2 source for intense cold neutron beam prod ction at 'he ESS, in: arXiv physics eprints, No. arXiv:1401.6003, 2014.

[33] D. Milstead, A new 'igh se' sitivity search for neutron-antineutron oscillations at the ESS, arXiv:1510.01569v1

[34] L. Zanini, F. זezei, K. Batkov, E. Klinkby and A. Takibayev, General use of low-dimensional moderators in neutron sources, Jourı zl of Phy ics Conference Series 1021(1):012009, DOI: 10.1088/1742-6596/1021/1/012009, 2018 
Table 1. Variation of the beamport-averaged brightness $B_{0-20}$ on the top moderator a a fi : in of different moderator or fast neutron reflector configurations below the target.

\begin{tabular}{|c|c|}
\hline configuration & gain factor \\
\hline two moderators & 1 \\
Be $10 \%$ water & 1.09 \\
$\mathrm{~Pb}$ & 1.20 \\
$\mathrm{~W} 22 \% \mathrm{He}$ & 1.18 \\
Steel $10 \%$ water & 1.10 \\
Steel $20 \%$ water & 1.07 \\
$\mathrm{Cu} 20 \%$ water & 1.09 \\
\hline
\end{tabular}

Table 2. Time-average integral t ern ll and cold brightness in $\left[\mathrm{n} / \mathrm{cm}^{2} / \mathrm{sr} / \mathrm{s}\right]$ for 5 MW average beam power of different moderator concepts. ESS peak . iof ness are 25 times the indicated numbers. In the last column the ILL Yellow Book brightness is listed [4].

\begin{tabular}{|ccccccc|}
\hline & TDR & P $j$ ne .r & PC far & BF1 & BF2 & ILL YB \\
\hline opening & $4 \times 60^{\circ}$ & $2 \times 10^{\circ}$ & $2 \times 120^{\circ}$ & $2 \times 120^{\circ}$ & $2 \times 120^{\circ}$ & \\
$B_{20-100}$ & $1.06 \times 11^{13}$ & $1.6 \times 10^{13}$ & $2.21 \times 10^{13}$ & $2.47 \times 10^{13}$ & $2.60 \times 10^{13}$ & $6.7 \times 10^{13}$ \\
$B_{0-20}$ & $1.98 \times 10^{13}$ & $5.23 \times 10^{13}$ & $4.83 \times 10^{13}$ & $5.45 \times 10^{13}$ & $4.94 \times 10^{13}$ & $7.5 \times 10^{12}$ \\
\hline
\end{tabular}

